\title{
Dynamics on and of Complex Networks
}

by

\author{
Chris Varghese \\ Department of Physics \\ Duke University
}

Date:

Approved:

Joshua E. S. Socolar, Supervisor

\begin{tabular}{c}
\hline Rick Durrett, Advisor \\
\hline Ashutosh V. Kotwal \\
\hline
\end{tabular}

Robert P. Behringer

Dissertation submitted in partial fulfillment of the requirements for the degree of Doctor of Philosophy in the Department of Physics in the Graduate School of Duke University

2014 


\title{
$\underline{\text { ABSTRACT }}$
}

\section{Dynamics on and of Complex Networks}

by

\author{
Chris Varghese
}

Department of Physics

Duke University

Date:

Approved:

\begin{tabular}{c}
\hline Joshua E. S. Socolar, Supervisor \\
\hline Rick Durrett, Advisor \\
\hline Ashutosh V. Kotwal \\
\hline Robert P. Behringer
\end{tabular}

An abstract of a dissertation submitted in partial fulfillment of the requirements for the degree of Doctor of Philosophy in the Department of Physics in the Graduate School of Duke University 2014 
Copyright (c) 2014 by Chris Varghese All rights reserved except the rights granted by the Creative Commons Attribution-Noncommercial Licence 


\section{Abstract}

Networks - abstract objects composed of vertices connected by edges, are ubiquitous in the real world. Examples such as social networks, the world wide web, and neural networks in the brain are constantly evolving in their topology, the state of their vertices, or a combination of the two. This dissertation presents a computational and theoretical study of three models of network dynamics, one corresponding to each of these modes of evolution.

The first study models the disintegration of a social network of voters with binary opinions, who prefer to be connected to others with the same opinion. We study two versions of the model: the network evolves by voters in discordant ties choosing to either adopt the opinion of their neighbors, or to rewire their ties to some randomly chosen voter of (i) the same, or (ii) any, opinion. We examine how the probability of rewiring, and the initial fraction $\rho_{\mathrm{i}}$ in the minority, determine the final minority fraction $\rho_{\mathrm{f}}$, when the network has bifurcated. In case (i), there is a critical probability, that is independent of $\rho_{\mathrm{i}}$, above which $\rho_{\mathrm{f}}$ is unchanged from $\rho_{\mathrm{i}}$, and below which there is full concensus. In case (ii), the behavior above the critical probability, that now depends on $\rho_{\mathrm{i}}$, is similar; but below it, $\rho_{\mathrm{f}}$ matches the result of starting with $\rho_{\mathrm{i}}=1 / 2$. Using simulations and approximate calculations, we explain why these two nearly identical models have such dramatically different behaviors.

The second model, called the quadratic contact process (QCP) involves "birth" and "death" events on a static network. Vertices take on the binary states occu- 
pied(1) or vacant(0). We consider two versions of the model - Vertex QCP, and Edge QCP, corresponding to birth events $1-0-1 \longrightarrow 1-1-1$ and $1-1-0 \longrightarrow 1-1-1$ respectively, where '-' represents an edge. We study the fraction of occupied vertices at steady state as a function of the birth rate, keeping the death rate constant. To investigate the effects of network topology, we study the QCP on homogeneous networks with a bounded or rapidly decaying degree distribution, and those with a heavy tailed degree distribution. From our simulation results and mean field calculations, we conclude that on the homogeneous networks, there is a discontinuous phase transition with a region of bistability, whereas on the heavy tailed networks, the transition is continuous. Furthermore, the critical birth rate is positive in the former but zero in the latter.

In the third study, we propose a general scheme for spatial networks evolving in order to reduce their total edge lengths. We study the properties of the equilbria of two networks from this class, one of which interpolate between two well studied objects: the Erdős-Rényi random graph, and the random geometric graph. The first of our two evolutions can be used as a model for a social network where individuals have fixed opinions about a number of issues and adjust their ties to be connected to people with similar views. The second evolution which preserves the connectivity of the network has potential applications in the design of transportation networks and other distribution systems. 


\section{Contents}

Abstract $\quad$ iv

List of Tables $\quad$ ix

List of Figures $\quad$ x

List of Abbreviations and Symbols xiii

Acknowledgements $\quad$ xvi

1 Introduction 1

1.1 Complex Networks . . . . . . . . . . . . . . . 1

1.2 Basic network theory . . . . . . . . . . . . . . 5

1.3 Some network terminology . . . . . . . . . . . . . . . 7

1.4 Random graphs . . . . . . . . . . . . . . . . . . . . . . . . . . . . 9

1.4.1 The thermodynamic limit .............. . . 9

1.4.2 General uncorrelated random graphs . . . . . . . . . . . . . 10

1.4.3 The Erdös-Rényi random graph . . . . . . . . . . . . . . 11

1.4.4 The Configuration model . . . . . . . . . . . . 15

1.4.5 Power law random graphs . . . . . . . . . . . . . 16

1.5 Stochastic processes with random networks . . . . . . . . . . . 17

1.6 Phase transitions involving networks . . . . . . . . . . . . . . . . 19

1.6.1 Equilibrium vs. non-equilibrium systems . . . . . . . . . 20

1.6.2 Approximate analytical methods . . . . . . . . . . . 21 
1.7 Overview of the projects . . . . . . . . . . . . . . 22

2 Graph Fission in an Evolving Voter model $\quad 27$

2.1 Holme-Newman Model . . . . . . . . . . . . . . . . . . 28

2.2 Our Model \& Simulation Results . . . . . . . . . . . . . . . . . 29

2.3 Quasi-stationary distributions ... . . . . . . . . . . 32

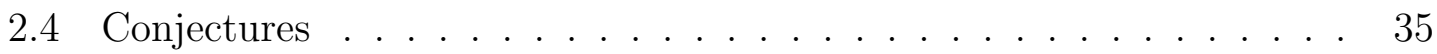

2.5 Discussion . . . . . . . . . . . . . . . . . . . 39

3 The Quadratic Contact Process on Complex Networks 41

3.1 Introduction . . . . . . . . . . . . . . . . . . . . . . 41

3.2 Model definition . . . . . . . . . . . . . . . . . . . 43

3.3 Mean field calculations . . . . . . . . . . . . . . . 45

3.3.1 Homogeneous networks . . . . . . . . . . . . . . 46

3.3.2 Heavy tailed degree distributions . . . . . . . . . . . . . . . 48

3.4 Some rigorous results . . . . . . . . . . . . . . . . . . 53

3.5 Simulation results . . . . . . . . . . . . . . . . 55

3.6 Conclusion . . . . . . . . . . . . . . . . . . . 59

4 An Evolving Spatial Network model $\quad 62$

4.1 Introduction . . . . . . . . . . . . . . . . 62

4.1.1 A general evolution scheme ............. 63

4.1.2 Our model . . . . . . . . . . . . . . . . . . . . 65

4.2 The unconstrained network .............. 66

4.2.1 A Fermion gas picture and connection with percolation . . . . 66

4.2.2 Properties of the percolation network . . . . . . . . . . . 67

4.2.3 A model for a social network . . . . . . . . . . . . . 75

4.3 The Connected ESNM . . . . . . . . . . . . . 76 
4.3.1 The $\beta \rightarrow \infty$ model as an optimization process . . . . . . . . 77

4.3.2 Other properties .................. 81

4.3.3 Testing the model on real data . . . . . . . . . . . . 83

4.4 Summary and Outlook . . . . . . . . . . . . . . . . 87

$\begin{array}{llr}5 & \text { Closing remarks } & 89\end{array}$

$\begin{array}{ll}\text { Bibliography } & 91\end{array}$

$\begin{array}{ll}\text { Biography } & 103\end{array}$ 


\section{List of Tables}

1.1 Examples of complex networks (adapted from page 110 of [1]). . . . 2

3.1 Nature of phase transitions of contact processes on various networks. Note that ' 0 ', '+' and ' $\infty$ ' stand for zero, positive and infinite values respectively of $\lambda_{c}$. The superscripts $\mathfrak{r}, \mathfrak{s}$, and $\mathfrak{m}$ indicate how the corresponding result was obtained - rigorously, by simulation, and by mean field calculation, respectively. . . . . . . . . . . . 60

4.1 Comparison of various statistics of the actual and simulated networks. 83

4.2 Statistics obtained for the ESNM on US state capitals' locations with three values of the mean degree. . . . . . . . . . . . . 86 


\section{List of Figures}

1.1 Fraction of vertices $\rho$ in the giant component as a function of the mean degree $\mu$ for the Erdös-Rényi random graph. . . . . . . . . . . . . . . 13

2.1 Simulation results for rewire to same model, starting from Erdös-Rényi graphs with $n=100,000$ nodes and average degree $\mu=4 . \quad$. . . . . 30

2.2 Simulation results for the rewire to random model, starting from Erdös-Rényi graphs with $n=100,000$ nodes and average degree $\mu=4$. 31

2.3 Fraction of nodes with the minority opinion $\left(\min \left\{n_{0}, n_{1}\right\} / n\right)$ and the number of discordant edges $n_{10}$ versus time, for a simulation of $n=$ 1000 nodes, $\rho_{0}=0.5$, and $\alpha=0.3 \ldots \ldots$. . . . . . . . 33

2.4 Plot of $n_{10} / m$ versus $n_{1} / n$ when $\alpha=0.5$ in the rewire to random case. Five simulations starting from $\rho_{0}=0.2,0.35,0.5,0.65$, and 0.8 are plotted in different colors. These results are from graphs with $n=10,000$ vertices and plotted every 1,000 steps. . . . . . . . . . 34

2.5 Plot of $n_{010} / n$ versus $n_{1} / n$ when $\alpha=0.5$ in the rewire to random case. All simulations start at $\rho_{0}=0.5$, since multiple runs from one starting point are enough to explore all of the arch. These results are from graphs with $n=100,000$ vertices and plotted every 10,000 steps. 36

2.6 Observed arches for the rewire to random model. The specified parabolas are fits to simulation data with $n=10,000, \mu=4 \ldots . . . . .37$

2.7 Observed arches for rewire to same model. The specified parabolas are fits to simulation data with $n=10,000, \mu=4 . \quad \ldots . . . \quad 38$

3.1 The solid red (top), dashed and solid blue (bottom) curves correspond to $\rho_{*}=\rho_{+}, \rho_{-}$and 0 respectively obtained from the mean field calculation for both QCP types on homogeneous networks. . . . . . . . . . 47

$3.2 I(\lambda, \theta)$ versus $\theta$ near $\lambda=\lambda_{c}$ for various power law graphs. . . . . . . . 50 
3.3 Steady state density reached, starting from all vertices occupied, for QCP on homogeneous networks of various sizes $n \ldots \ldots \ldots \ldots$. . . . . . . . .

3.4 Steady state density reached, starting from all vertices occupied, for QCP on power law networks of various sizes $n$. Note that the $\lambda$ axis is in the $\log$ scale. . . . . . . . . . . . . . 57

3.5 Steady state density reached, starting from two different initial densities $\rho^{(0)}$, for QCP on homogeneous networks of size $n=10^{5}$. Notice the similarity with the mean field prediction on Fig. 3.1. . . . . . . 58

3.6 Steady state density when the birth rate is infinite in the VQCP on various networks of size $n=10^{5}$. Note that the $\rho^{(0)}$ axis is in the log

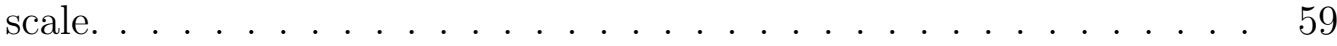

4.1 Results for $\beta=1$ (solid line), 3 (dashed line), and $\infty$ (dotted line) in dimension $D=2$; (a) gives the distribution of edge lengths when $\mu=5$, (b) gives the mean edge length as a function of $\mu \ldots \ldots 70$

4.2 Pictures of the (a) Erdős-Rényi graph, and (b) the random geometeric graph, on a square with $n=1000$ vertices. . . . . . . . . . . 71

4.3 Fraction of vertices $\rho$ in the largest component (a) as a function of $\mu$ for various values of $\beta$ and (c) as a function of $\beta$ for various values of $\mu$. (b) shows the finite size scaling for $\beta=3$; notice that all the curves seem to cross at one point. . . . . . . . . . . . . . . . 74

4.4 Clustering coefficient as a function of $\mu$ for various values $\beta$. (The non-uniform distribution of data points in $\mu$ correspond to uniform data points in $\kappa_{\beta \mu}^{(D)}$.) $\ldots \ldots \ldots \ldots \ldots \ldots \ldots \ldots \ldots$

4.5 A realization of the almost optimized network $(\beta=10)$ for two different values of the mean degree. The red circles and blue lines correspond to the vertices and edges respectively $\ldots \ldots \ldots . \ldots 76$

4.6 The route factor $R$ as a function of the (a) mean degree $\mu$, and (b) the total edge length per vertex $\chi$, for the RCN and AON. . . . . 80

4.7 Robustness of the (a) RCN and (b) AON. $\ldots \ldots \ldots \ldots$

4.8 Degree distribution of the random connected and optimized networks. 81

4.9 Mean edge length $\xi$ as a function of $\mu$ for the AON . . . . . . . . 81

4.10 Clustering coefficient as a function of the mean degree $\mu \ldots \ldots . . .82$ 
4.11 Hop distance $h$ and route distance $r$ as a function of the mean degree $\mu$ for the RCN and AON. . . . . . . . . . . . . . . . . . . 82

4.12 Pictures generated using the Minnesota road network data - (a) the actual network, and (b) the simulated network with $\beta=50 . \quad$. . . . 83

4.13 Comparison of (a) robustness and (b) degree distribution, of the actual, simulated, and Erdős-Rényi networks. . . . . . . . . . . . . . . . 84

4.14 Pictures of connected ESNM on US the state capitals" locations for two values of the mean degree. . . . . . . . . . . . . . . . 85

4.15 Comparison of (a) robustness and (b) degree distribution, of simulation results for US state capitals' locations data for three values of

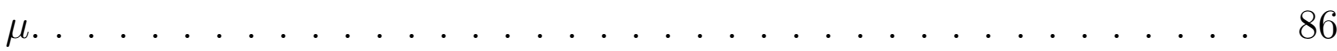




\title{
List of Abbreviations and Symbols
}

Symbols

\author{
$\mathbb{P}(x)=$ probability of event $x$ \\ $\mathbb{E}(x)=$ expected value of $x$ \\ $\langle x\rangle=$ expected number of copies of a small labeled graph $x$ \\ contained in given graph \\ $\mathbb{1}_{x}=1$ if condition $x$ true,$=0$ otherwise \\ $\left(\begin{array}{l}x \\ y\end{array}\right)=x ! / y !(x-y) !$ \\ $\operatorname{Binomial}(x, y ; z)=\left(\begin{array}{l}x \\ z\end{array}\right) y^{z}(1-y)^{x-y}$ \\ $\operatorname{Poisson}(x ; y)=e^{-x} x^{y} / y$ ! \\ $|x|$ means absolute value / Euclidean norm / cardinality, if $x$ is \\ a number / vector / set \\ $f(x)=\mathcal{O}(g(x))$ means $\exists M:|f(x)| \leq M|g(x)|, \forall x$ \\ $x \approx y$ means $x$ is approximately equal to $y$ \\ $x \rightarrow y$ means $|x-y|<\epsilon, \forall \epsilon>0$ \\ $x_{k} \sim y_{k}$ means $x_{k} / y_{k} \rightarrow$ constant as $k \rightarrow \infty$ \\ $x_{k} \lesssim y_{k}$ means $\exists c>0: x_{k} / y_{k}<c \forall k$ \\ C clustering coefficient \\ D dimension \\ $d \quad$ degree \\ $d^{(2)} \quad$ secondary degree $=$ number of second neighbors
}




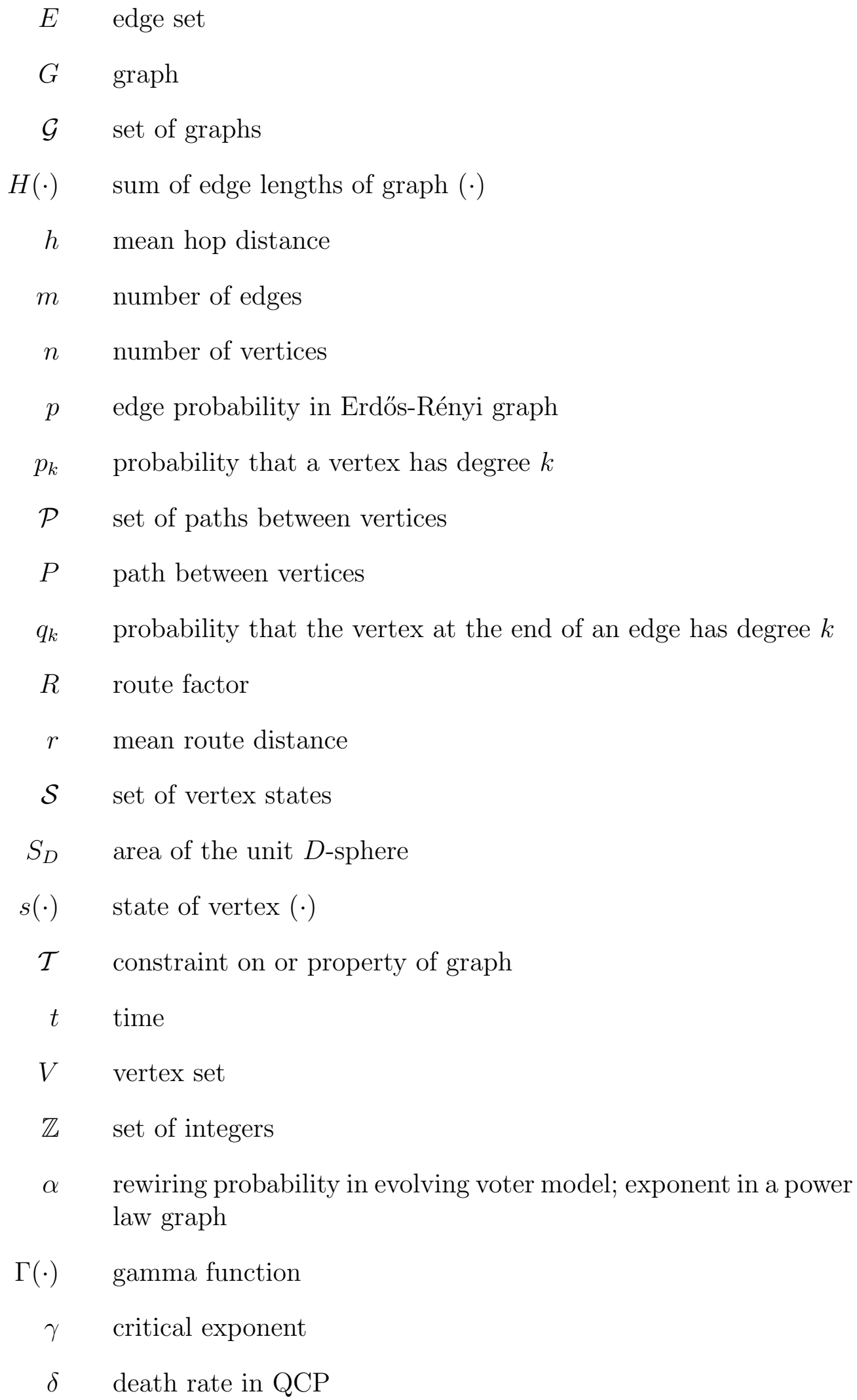


$\Xi \quad$ grand partition function

$\mu \quad$ mean degree

$\nu \quad=\mathbb{E}\left[d^{(2)}\right] / \mathbb{E}[d]$

$\xi \quad$ mean edge length

$\chi \quad$ total edge length per vertex $=\xi \mu / 2$

$\Omega_{D} \quad$ volume of unit $D$-ball

$\rho_{*} \quad$ order parameter; size of giant component

$\boldsymbol{\pi}$ probability distribution of random graph

$\lambda \quad$ birth rate in $\mathrm{QCP}$

\section{Abbreviations}

i.i.d independent and identically distributed

MFA mean field approximation

ER Erdős-Rényi random graph

PL power law random graph

RR random regular graph

GC giant component

CP contact process

QCP quadratic contact process

VQCP vertex quadratic contact process

EQCP edge quadratic contact process

ESNM evolving spatial network model

RGG random geometric graph

MST minimum spanning tree

RCN random connected network

AON almost optimized network 


\section{Acknowledgements}

First and foremost I thank my advisor Prof. Rick Durrett for agreeing to advise me, especially considering that I am a graduate student in a department (Physics) different from his (Mathematics). I am also thankful to Prof. Josh Socolar for agreeing to be my co-advisor in Physics, taking valuable time out of that meant for his primary research commitments. I would also like to thank the previous and current Directors of Graduate Studies Prof. Richard Palmer and Prof. Shailesh Chandrasekharan respectively, for their guidance.

Research grants are extremely competitive, making it a scarce and valuable resource, especially in the pure sciences. I am extremely grateful to Prof. Durrett for providing me full funding ${ }^{1}$ (that covered my tuition and other fees, and stipend) for a major part of my time at Duke, and partial funding for attending Dynamic Days 2013, and the APS March Meeting 2013. I am also grateful to Professors Jim Moody (Duke Sociology) and Peter Mucha (UNC Mathematics) for funding me from their joint grant ${ }^{2}$ during spring 2014 . Thanks also to the Duke Graduate school for funding me during summer 2012.

I take this opportunity to thank Dr. Mark Novotny (MSU) and Dr. Stefan Boettcher (Emory) for introducing me to the exciting field of networks that this dissertation focuses on, and Prof. Dibyendu Das (IIT Bombay) for helping me identify my research interests during my undergraduate days. Thanks are also due to past

${ }^{1}$ NSF grants DMS 1005470 and 1305997

21 R01 HD075712-01 
graduate students Abhijit Mehta and Christopher Coleman-Smith for introducing me to some effective ways to manage large computational projects in Linux. Thanks to officemates Cate Marcoux, Sam Gong, and Mengyang Sun for stimulating and friendly discussions in our common windowless office room.

It would be understatement to say that my pet dog Marley had a big role to play in the completion of this dissertation. Although he was not much of a help with the research per se, I thank him for being my constant companion during the ups, and more importantly, the downs of my graduate school life at Duke.

I would also like to acknowledge my family and friends whose moral and emotional support was vital to the completion of this dissertation: niece Evalyn Rema, sister Sumy Joy, brother-in-law Gireesh Rema, Dad, friends Sreepriya Pramod, Dvijotham Krishnamurthy, Prakash Kumar Nayak, Marco Bertolini, David Rosin, Lynn Kaack, and many others.... 


\section{Introduction}

The purpose of this chapter to provide the reader with all the background information needed to comprehend the material in Chapters 2, 3, and 4. First we introduce the relatively new and emerging field of complex networks that is the focus of this dissertation. We then review the basics of network theory and some of the associated terminology. We then proceed to a general description of random graphs that are used as models of complex networks, and the stochastic processes involving them and their associated phase transitions. Since all these topics are extremely broad, we will restrict our discussion to parts of them that are relevant to the dissertation. Finally, we give an overview of the three projects covered in the later chapters that form the core of this dissertation.

\subsection{Complex Networks}

A wide variety of systems, both natural and man-made, could be viewed simply as a collection of entities that interact with each other. The entities themselves may have a complex internal structure; but their relevant behavior, i.e., how they interact with their peers, is usually relatively simple. Yet, the system as a whole exhibits what 
are called emergent phenomena. By this we mean that the behavior is non-intuitive and difficult to predict solely from the knowledge of the system's entities. In other words, the interaction between the different entities is the key to their macroscopic behavior.

Examples of the aforementioned systems include many biological systems such as the brain where the interaction between the neurons lead to "consciousness" [2], and large social groups where interactions among individuals lead to the spread of an epidemic, or the formation of an opinion on an issue.

All the above examples entice us to abstract them as a network or graph - a mathematical object composed of vertices and edges, the former corresponding to the entities, and the latter to the interactions. Furthermore, these networks may be more approriately called complex networks, since their topologies are typically far from regular (as in, say, a square lattice) but at the same time, not completely random either; in other words the connection between vertices do not have an easily discernible pattern, which makes them intriguing and fascinating. For example, the world wide web (WWW) connecting billions of web pages through hyper links, although a man-made creation, has a very complex structure that is constantly evolving and is yet to be fully understood.

Table 1.1: Examples of complex networks (adapted from page 110 of [1]).

\begin{tabular}{lll}
\hline Network & Vertex & Edge \\
\hline Internet & Computer or router & Cable \\
Citation network & Article, patent, or legal case & Citation \\
Power grid & Generating station or substation & Transmission line \\
Friendship network & Person & Friendship \\
Metabolic network & Metabolite & Metabolic reaction \\
Neural network & Neuron & Synapse \\
Airline network & Airport & Flight segment \\
Road network & Cities & Road \\
Food web & Species & Predation \\
\hline
\end{tabular}

Complex networks may show properties such as 
- the number of hops needed to travel between any two vertices being much smaller than the network size. This is also called the small world property leading to the notion of "six degrees of separation".

- the presence of vertices called hubs that have an unusually large number of edges connected to them. For example, in the world wide web (WWW), the number of hyper links to google.com is many orders of magnitude larger than that to most sites in the WWW [3]. Such networks are said to have a heavy tailed degree (number of neighbors) distribution.

- community structure, i.e., the tendency of groups of vertices to have significantly more connections among themselves than to vertices outside the group. For example, consider the friendship network of high school students. There could be cliques of students who "hang out" together with few interactions between students belonging to different groups, and thereby dividing the friendship network into communities. Designing algorithms for the detection of communities in a network is a very active research area [4].

- high transitivity or clustering, i.e., the tendency of the neighbors of a vertex to be connected to each other. In a social network one's friends are highly likely to be also friends of each other.

- assortativity or homophily, i.e., the tendency of vertices with similar attributes to be connected to each other, or the opposite tendency called dissortativity. In a social network, for example, individuals may have a tendency to preferentially form friendships with others of similar age, national origin, income level, etc.

Notice that the features mentioned above are in stark contrast to those of regular lattices that have traditionally been studied in physics; in other words, lattices are not complex networks. It is also important to differentiate the field of complex 
networks from that of graph theory which has a long history in discrete mathematics starting with Euler's famous solution of the Königsberg Bridge Problem in 1735. Graph theory typically deals with small graphs with relatively regular features and attempts to prove rigorous and exact results about them. Examples of problems considered in graph theory include existence of Hamiltonian paths, graph coloring (e.g.: the four color theorem), planarity and finding maximal independent sets.

The field of complex networks, on the contrary, is relatively new and highly interdisciplinary, and aims to describe, and uncover the large scale properties of, huge graphs found in the real world. Research on complex networks have primarily advanced in two complementary directions: the empirical study of real world networks on the one hand, and the use of simplistic abstract models that attempt to replicate the observed features, on the other. The latter includes the development of models, their analysis by simulation, by approximate calculations from heuristic arguments, and by rigorous mathematical results. This dissertation focuses on the first three, with simulations playing the major part.

The history of complex networks may be dated back to the 1950s. During this period, political scientist Ithiel de Sola Pool and mathematician Manfred Kochen became interested in social networks, especially the small world phenomenon [5]. In the same decade, mathematicians Solomonoff and Rapoport [6], and Paul Erdös and Alfréd Rényi [7] undertook pioneering studies on random graph models. Their models which have now come to be know as the Erdős-Rényi random graph (ER) was studied extensively for many decades that followed. The ER graph did have the small world property. However, it failed to reproduce two other important features: the high clustering seen in social networks, and the scale free (power law) degree distribution seen in many real world networks.

The empirical study of real networks was not very active until the late 1990s when technological developments lead to the ease of access to and computational 
power to process, large data sets about them. Two seminal papers by Watts and

Strogatz [8], and Barabási and Albert [9] triggered an explosion in the number of publication on complex networks $[1,10]$. The former paper introduced what is now known as the Watts-Strogatz or WS model, which generated networks that in addition to the small world property showed high clustering. Notice that the properties of small world, and high transitivity, are in some sense at odds with each other. It is therefore remarkable that real networks have this property, and the WS model was able to capture it. The latter paper proposed a "preferential attachment" growth model, i.e., new vertices connect preferentially to existing vertices of high degree, for networks. This model, that has come to be know an the Barabási-Albert or BA model, generated networks that had a power law degree distribution, and small values for the diameter (maximum distance between vertex pairs), but had very low clustering.

\subsection{Basic network theory}

A network, also called a graph, $G$ is specified by its vertices and its edges. Mathematically, a network is a pair $G=(V, E)$, where $V$ is the set of vertices and $E$ is the set of edges. We will denote the number of vertices and edges by $n=|V|$, and $m=|E|$, respectively. In the most general type of network, the edges are directed, and are also allowed to point from a vertex to itself forming a self loop. In this dissertation, however, we will limit ourselves to undirected edges and disallow self loops. Such networks are called simple graphs and here the edge set is composed of unordered pairs of distinct elements the vertex set, i.e., $E \subseteq\left(\begin{array}{c}V \\ 2\end{array}\right) \equiv\{\{x, y\}: x, y \in V, x \neq y\}$. However, it may sometimes be more convenient to view an undirected network as a directed one by simply viewing each undirected edge to be composed of two directed edges pointing in opposite directions.

The vertex set and edge set together specify the topology of the network. How- 
ever, real world networks have a lot more structure to them. Typically, the vertices of a network have states associated with them. For example, in a social network where the vertices represent individuals, and the edges represent friendship between pairs of individuals, the state of the vertices could be the opinion of the individuals on a particular issue. Thus, the full specification of the network may require the inclusion of the vertex states. Additionally, networks in the real world are hardly ever static. Our friends change, opinions change, we get infected by diseases, and so on. In other words, the networks associated with real world systems evolve over time.

If $\mathcal{S}$ is the set of states that vertices can adopt, then we can represent the graph by the triple $G_{t}=\left(V_{t}, E_{t}, s_{t}\right)$, where $s_{t}: V_{t} \rightarrow \mathcal{S}$. If no two vertices can be in the same state, we can use the states of the vertices to label them, eliminating the need for $s$.

The above mentioned simplification could be done for spatial networks - a special class of networks, whose vertices are embedded in space. To be precise, the vertex set of spatial networks is a set of points in a metric space, so that there is a notion of distance between every vertex pair. The state a vertex is its spatial location, and is unique if we disallow any two vertices to be in the same location.

If $V_{t}, E_{t}$ and $s_{t}$ are all constant then the network is static. In all other cases the network is dynamic. If $V_{t}$ and $E_{t}$ are constant and $s_{t}$ alone changes, then we call the process a dynamics on the network. On the other hand, if $s_{t}$ is fixed, but $V_{t}$ and/or $E_{t}$ change, then it is termed dynamics of networks. The more general case where the topology of networks and the state of vertices change is the realm of coevolving networks.

In this dissertation, we will treat time to be continuous as it is in the real world. Additionally, we restrict ourselves to networks whose set of vertices, and number of edges do not change, i.e., $V_{t}$ and $\left|E_{t}\right|=m$ are constant in time. 


\subsection{Some network terminology}

The triple $(V, E, s)$ completely specifies the network. However, for large networks, this level of detail is usually impractical and unnecessary. Instead, we introduce quantities that provide a more insightful global description of the network. We will first define some terms at the local level, and use them to build the terms for the global description.

If there is an edge between two vertices then each of the vertices is called a neighbor of the other, i.e., if $\{x, y\} \in E$ then $x$ is a neighbor of $y$ and vice-versa. The number of neighbors that a vertex has is called its degree $d$, i.e., $d(x)=\mid\{y:\{x, y\} \in$ $E\} \mid$. The average degree $\mu=\sum_{x} d(x) / n$ of the vertices is one of the simplest global measures of the network. It is easy to see that $\mu=2 m / n$. A more informative description of the network is given by the distribution $p_{k}=|\{x: d(x)=k\}| / n$ of

the degrees. A network where every vertex has the same degree, i.e., $p_{k}=\mathbb{1}_{k=\mu}$, is called a regular graph. Related to the degree distribution is the size-biased degree distribution $q_{k}$, which is the degree distribution of the neighbors of a vertex, i.e., $q_{k}=|\{(x, y):\{x, y\} \in E, d(y)=k\}| / 2 m$. It can be seen that the two distributions are related as $q_{k}=k p_{k} / \mu$.

A subgraph $G^{\prime}=\left(V^{\prime}, E^{\prime}\right)$ of a graph $G=(V, E)$, is a graph that contains some (or all) of the vertices of $G$ and all the edges connecting those vertices, i.e., $G^{\prime} \subseteq$ $G \Rightarrow V^{\prime} \subseteq V, E^{\prime}=E \cap\left(\begin{array}{c}V^{\prime} \\ 2\end{array}\right)$.

The $p_{k}$ and $q_{k}$ distributions deals with vertices and their immediate neighborhood. Moving beyond that, consider the set of paths

$$
\mathcal{P}(x, y)=\left\{\left(x, z_{1}, z_{2}, \ldots, z_{\ldots}, y\right):\left\{x, z_{1}\right\},\left\{z_{1}, z_{2}\right\}, \ldots,\left\{z_{\ldots}, y\right\} \in E\right\}
$$

between two vertices $x$ and $y$. If a path exists between every pair of vertices, then we say that the network is connected. In general, however, this may not be the case and the network could be composed of disconnected components. A component $G^{\prime}$ 
of a graph $G$ is a maximal subgraph of $G$ that is connected, i.e., it is impossible to add more vertices to $G^{\prime}$ while still maintaining the connectivity property.

For a path $P \in \mathcal{P}(x, y)$, the hop length $h(P)$ is the number of edges in $P$. In a spatial network, one can also define a route length $r(P)=\left|x-z_{1}\right|+\left|z_{1}-z_{2}\right|+$ $\ldots+\left|z_{\ldots}-y\right|$. Related to these path lengths, are two kinds of distances between $x$ and $y$ : the hop distance $h(x, y)=\min _{P \in \mathcal{P}(x, y)} h(P)$, and the route distance $r(x, y)=$ $\min _{P \in \mathcal{P}(x, y)} r(P)$. Three related global metrics are the mean hop distance $h$, the mean route distance $r$, and the largest hop distance among all vertex pairs, called the diameter. Note that in a disconnected network, the definitions above result in an infinite value for the three global metrics, and therefore in such cases, one considers only the largest component of the network.

A cycle or loop is a path from a vertex to itself. A graph that does not contain any cycles is a called a tree. It is easy to see that any tree on $n$ vertices will have exactly $n-1$ edges. A connected network that is also a tree is called a spanning tree. In spatial networks (or more generally, in networks with weighted edges), the spanning tree with the smallest total edge length (edge weight) is called a minimum spanning tree. In many approximate calculations on networks it is common to treat them to be locally tree like. This means that if one starts from a vertex and follows its neighbors and their neighbors and so on, then the first cycle is encountered after a large number of hops.

As mentioned earlier, in many real networks, especially social networks, there is a tendency for the neighbors of a vertex to be connected to each other. This property called transitivity is quantified by a metric called the clustering coefficient defined as

$$
C=\frac{\text { number of triangles } \times 6}{\text { number of paths of hop length two }} \text {. }
$$




\subsection{Random graphs}

We argued earlier that real world networks are complex. We seek to capture this complexity in our network models by making them as random as possible, subject to a handful of reasonable constraints.

A random graph, similar to a random variable, is defined by its probability distribution $\boldsymbol{\pi}$ over the set $\mathcal{G}$ of all graphs, i.e., $\boldsymbol{\pi}: \mathcal{G} \rightarrow[0,1]$.

\subsubsection{The thermodynamic limit}

As is common in the literature, in the network models we study, we will be interested in the limit where the network size $n \rightarrow \infty$, also known as the thermodynamic limit. Furthermore, the networks we study will be sparse, i.e., the number of edges $m=\mathcal{O}(n)$, or equivalently, the mean degree $\mu=\mathcal{O}(1)$.

For random graphs in the thermodynamic limit, some of the network metrics we discussed in Section 1.3 could be slightly redefined. If $x$ is a randomly chosen vertex, the degree distribution $p_{k}=\mathbb{P}(d(x)=k)$ and the mean degree $\mu=\mathbb{E}[d(x)]$. If $(x, y)$ is a randomly chosen directed edge, then $q_{k}=\mathbb{P}(d(y)=k)$. The clustering coefficient is the probability that two vertices that are connected to a common third vertex are also connected to each other, i.e., for three randomly chosen vertices $x, y$ and $z$,

$$
C=\mathbb{P}(\{x, z\} \in E \mid\{x, y\},\{y, z\} \in E) .
$$

An important concept that arises in the thermodynamic limit is that of the giant component (GC). For a random network with $n$ vertices, the giant component is a component, if there exists one, whose size is proportional to $n$, i.e., if the GC contains $n_{\mathrm{GC}}$ vertices, then

$$
\lim _{n \rightarrow \infty} \frac{n_{\mathrm{GC}}}{n}=\text { constant. }
$$




\subsubsection{General uncorrelated random graphs}

The simplest types of random graphs are degree uncorrelated random graphs, which means that given two connected vertices $x$ and $y$, their degrees $d(x)$ and $d(y)$ are independent random variables. The topology of these networks are fully determined by their degree distribution. The initial state of all the random graphs studied in this dissertation will have this property. The Erdős-Rényi random graph and the more general configuration model discussed later are all examples of uncorrelated random graphs.

We will now investigate the conditions under which uncorrelated networks will have a giant component. Let $x$ be a randomly chosen vertex and let $\rho$ be the probability that $x$ belongs to the giant component, and $\omega$ be the probability that a randomly chosen neighbor $y$ of $x$ does not belong to the giant component if the edge $\{x, y\}$ is deleted from the network. The number of neighbors of $y$ is distributed according to the size-biased degree distribution $q_{k}$. Now, $y$ is connected to the GC solely through $x$ iff all its neighbors, except $x$, are connected to the GC through $y$. This means

$$
\omega=\sum_{k=1}^{\infty} q_{k} \omega^{k-1}=g_{q}(\omega)
$$

The number of neighbors of $x$ are distributed according to $p_{k}$ and $x$ is not part of the GC iff none of its neighbors are part of the GC, i.e.,

$$
1-\rho=\sum_{k=0}^{\infty} p_{k} \omega^{k}=g_{p}(\omega) .
$$

Functions like $g_{p}$ and $g_{q}$ are called the generating functions of the corresponding

probability distributions. Solving (1.4) for $\omega$ and plugging it in (1.5), one can find the fraction $\rho$ of vertices in the giant component. (See [11] for a more detailed version of the calculation that follows) Notice that $g_{p}(1)=g_{q}(1)=1$ and therefore $\rho=0$ 
is a trivial solution. Also, notice that $g_{q}(0)=q_{1}$. A non-trivial solution exists if $g_{q}^{\prime}(1)>1$, i.e.

$$
g_{q}^{\prime}(1)=\sum_{k=1}^{\infty}(k-1) q_{k}=\sum_{k=1}^{\infty}(k-1) \frac{k p_{k}}{\mu}=\frac{\mathbb{E}\left[d^{2}\right]-\mathbb{E}[d]}{\mathbb{E}[d]}>1,
$$

Notice that the numerator $\mathbb{E}\left[d^{2}\right]-\mathbb{E}[d]=\mathbb{E}[d(d-1)]$ is the expected number of second neighbors $d^{(2)}$ of a vertex; therefore, (1.6) essentially means that the expected number of second neighbors must be greater than that for the (first) neighbors. Defining the branching ratio $\nu=\mathbb{E}\left[d^{(2)}\right] / \mathbb{E}[d]$, the condition for the existence of a giant component, which was first given by Molloy and Reed [12], is $\nu>1$.

What is the largest degree $d_{\max }=\max _{x \in V} d(x)$ in a network of size $n$, and is it "large", i.e., does the network contain hubs? In degree uncorrelated graphs, since the $d(x)$ 's are i.i.d, the distribution of $d_{\max }$ is, in principle, straight forward to write down:

$$
\begin{aligned}
\mathbb{P}\left(d_{\max }=j\right) & =[\mathbb{P}(d \leq j)]^{n}-[\mathbb{P}(d \leq j-1)]^{n} \\
& =\left[\sum_{k \leq j} p_{k}\right]^{n}-\left[\sum_{k \leq j-1} p_{k}\right]^{n}
\end{aligned}
$$

although, depending on $p_{k}$, calculating or even estimating, say, $\mathbb{E}\left[d_{\text {max }}\right]$ could be cumbersome. A quicker alternative to estimating $d_{\max }$ is to look at the "thickness of the tail" of $p_{k}$. Specifically, if $d_{n}=\min \{k: n \mathbb{P}(d>k)<1\}$, then $d_{\text {max }} \sim d_{n}$, which means that

$$
n \mathbb{P}\left(d>d_{\max }\right) \approx 1
$$

gives a good estimate of $d_{\max }$.

\subsubsection{The Erdös-Rényi random graph}

The simplest random graph, with $n$ vertices and mean degree $\mu$, one can think of is where the $m=n \mu / 2$ edges are chosen uniformly at random from all possible vertex 
pairs, i.e., $E$ is a random subset of $\left(\begin{array}{c}V \\ 2\end{array}\right)$ of size $m$. Such a random graph was studied extensively by Erdős and Rényi $[7,13,14]$ and is therefore named after them. Since

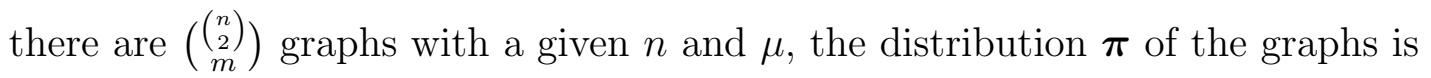

$$
\boldsymbol{\pi}(G)= \begin{cases}1 /\left(\begin{array}{c}
\left(\begin{array}{c}
n \\
2 \\
m
\end{array}\right) \\
0
\end{array}\right) & \text { if }|V(G)|=n,|E(G)|=m \\
0 & \text { otherwise }\end{cases}
$$

The above random graph model is sometimes called the $\mathcal{G}(n, m)$ model. Although $\mathcal{G}(n, m)$ is simple to define, it is easier to work with a slightly different version of $\mathcal{G}(n, m)$ called the $\mathcal{G}(n, p)$ model $[6,15]$. In the $\mathcal{G}(n, p)$ random graph, edges are created between vertex pairs with a fixed probability $p$ and so the distribution of graphs is

$$
\boldsymbol{\pi}(G)=\text { Binomial }\left[\left(\begin{array}{c}
n \\
2
\end{array}\right), p ;|E|\right]=\left(\begin{array}{c}
\left(\begin{array}{c}
n \\
2
\end{array}\right) \\
|E|
\end{array}\right) p^{|E|}(1-p)^{\left(\begin{array}{c}
n \\
2
\end{array}\right)-|E|}
$$

if $|V|=n$, and 0 otherwise. The properties of the $\mathcal{G}(n, m)$ and $\mathcal{G}(n, p)$ models approach each other in the $n \rightarrow \infty$ limit. Their relation is similar to that between the canonical and grand canonical ensembles in statistical mechanics. The mean number of edges in $\mathcal{G}(n, p)$ is $\left(\begin{array}{l}n \\ 2\end{array}\right) p$. If we set this equal to $m$, then the $\mathcal{G}(n, m)$ and $\mathcal{G}(n, p)$ random graphs have similar properties, since the density in the binomial distribution is strongly peaked around its mean when $n \rightarrow \infty$, i.e.,

$$
\left(\begin{array}{l}
n \\
2
\end{array}\right) p=n \frac{\mu}{2} \Rightarrow p=\frac{\mu}{n}
$$

Some properties of the Erdős-Rényi (ER) random graph are easy to calculate. Since the edges are all assigned independent of each other, the clustering coefficient

$$
C=\mathbb{P}(\{x, z\} \in E \mid\{x, y\},\{y, z\} \in E)=\mathbb{P}(\{x, z\} \in E)=\frac{\mu}{n} .
$$


This implies that the ER graph has $C \rightarrow 0$ in the $n \rightarrow \infty$ limit, and therefore is a poor model for most real networks, especially social networks, which have been found to possess significant clustering.

Since a vertex is connected to each of the other $n-1$ vertices with a fixed probability, the degree distribution

$$
\begin{aligned}
\mathbb{P}(d=k) & =\operatorname{Binomial}(n-1, p ; k) \\
& \rightarrow \operatorname{Poisson}(\mu ; k) \text { as } n \rightarrow \infty
\end{aligned}
$$

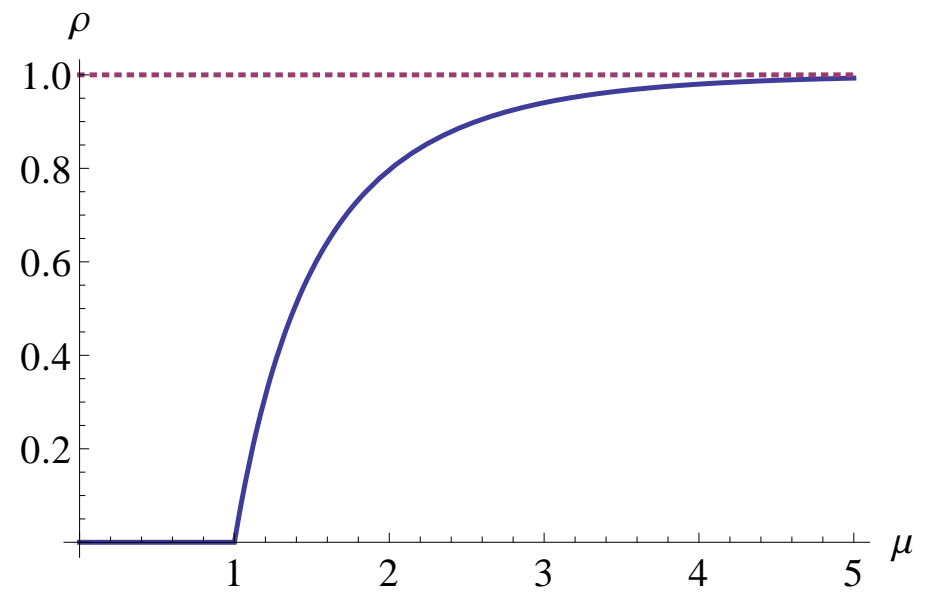

FiguRE 1.1: Fraction of vertices $\rho$ in the giant component as a function of the mean degree $\mu$ for the Erdős-Rényi random graph.

Solving equations (1.4) and (1.5), the size of the giant component can be found to be

$$
\rho=1+\frac{1}{\mu} W\left(-\mu e^{-\mu}\right)
$$

where $W(z)$ is the function that solves $z=W e^{W}$. The size of the largest component as a function of the mean degree is plotted in Fig. 1.1. The giant component emerges when $\mu=1$, consistent with the Molloy-Reed criterion. The formation of the giant component in an ER graph has strong similarities with the formation of a percolating cluster in bond percolation. More specifically, the Erdős-Rényi random graph on $n$ 
vertices is like bond percolation [16] on a Bethe lattice with coordination number $n-$ 1. The percolation threshold for a Bethe lattice is $p_{c}=1 /($ coordination number -1$)$, which for the ER graph becomes $1 /(n-2) \approx 1 / n$.

The size of the giant component increases with increasing $\mu$ until the graph becomes connected. When the graph is close to being connected, $\rho=1-1 / n$. Plugging this into (1.14), we get

$$
\begin{gathered}
W\left(-\mu e^{-\mu}\right)=-\frac{\mu}{n} \Rightarrow-\mu e^{-\mu}=-\frac{\mu}{n} e^{-\mu / n} \\
\Rightarrow \mu \sim \log n .
\end{gathered}
$$

When $\mu$ crosses $\log n$, we say that the ER graph undergoes a condensation transition, and becomes connected.

If one assumes the formation of an ER random graph to be a branching process [17], then one can get an order of magnitude estimate of the diameter or the mean hop distance $h$. The starting vertex has on average $\mu$ neighbors, and subsequent generations on average reproduce $\mu$ additional neighbors. Thus the number of expected number of vertices in the $\ell$-th generation is $\mu^{\ell}$. The total number of vertices in all generations is $n$ :

$$
\sum_{\ell=0}^{h} \mu^{\ell}=n \Rightarrow \frac{\mu^{h+1}-1}{\mu-1}=n \Rightarrow h \approx \frac{\log n}{\log \mu} .
$$

Thus the average hop distance is much smaller than the network size, and thus ER graphs are small world.

The tail of the Poisson distribution is thinner than that of an exponential distribution (i.e., $\exists K\left(\mu, \mu_{2}\right): \forall k>K$, Poisson $\left.(\mu ; k)<\operatorname{Exponential}\left(\mu_{2} ; k\right)\right)$. From (1.8), $d_{\text {max }}$ for the exponential distribution can be found to be $\mathcal{O}(\log n)$, implying that ER graphs have $d_{\max }=\mathcal{O}(\log n)$, and therefore are very short tailed. 


\subsubsection{The Configuration model}

One way to generate uncorrelated random graphs is by the configuration model introduced by Bender and Canfield [18] and further studied by Bollobás [19]. This model may be viewed as a generalization of the Erdős-Rényi graphs. While the original model was defined with a degree sequence, i.e., a degree specified for each vertex, Newman et al. [20] studied a more convenient version where the degree distribution is specified instead. This model generates random graphs that are maximally random subject to the given degree distribution.

The algorithm for generating a random graph by the configuration model is as follows. For each vertex $x$, we generate a random number $k_{x}$ from the given degree distribution. If $\sum_{x} k_{x}$ turns out to be odd, we decrease one of the $k_{x}$ 's by 1 to get $\sum_{x} k_{x}=2 m$. We then attach $k_{x}$ "half-edges" to $x$. Finally, we randomly pair all the half edges. The resulting graph clearly has the given degree distribution in the $n \rightarrow \infty$ limit.

However, there are a couple of caveats worth mentioning. First, some of the pairings could contain multiple edges and self loops. Fortunately, when $n \rightarrow \infty$, the number of self loops and the number of multiple edges can be shown (see Theorem 3.1.2. in [11]) to be independently distributed as Poisson $(\nu / 2)$ and Poisson $\left(\nu^{2} / 4\right)$, respectively, where $\nu$ is the branching ratio as defined earlier. This means that if the degree distribution has a finite second moment, then the expected number of self loops and multiple edges is $\mathcal{O}(1)$. Second, not all pairings lead to different graphs, i.e., given a pairing, permutations of the half-edges emanating from a common vertex, leave the graph unchanged. There are $(2 m-1) ! ! \equiv 1 \times 3 \times \ldots \times(2 m-1)$ ways to pair up the $2 m$ half-edges, each of which is equally likely to be chosen. Ignoring the negligible number of multiple edges and self loops, corresponding to any given pairing, there are $\prod_{x} k_{x}$ ! pairings, formed by permutations of the half edges, that 
produce the same graph. Putting this all together, the probability distribution in the configuration model is,

$$
\boldsymbol{\pi}(G) \approx \frac{1}{(2|E|-1) ! !} \prod_{x \in V} d(x) ! p_{d(x)} \text { if }|V|=n, \quad \text { and } 0 \text { otherwise }
$$

The probability of an edge between two vertices with degrees $k$ and $j$ respectively is $\mathbb{P}(\{x, y\} \in E \mid d(x)=k, d(y)=j) \approx k j / 2 m$. Suppose two vertices $x$ and $z$ are connected to a common vertex $y$, then $d(x)$ and $d(z)$ are distributed according to $q_{k}$, and so the probability of an edge between $x$ and $z$ gives the clustering coefficient to be

$$
C=\mathbb{P}(\{x, z\} \in E)=\sum_{k, j} q_{k} q_{j} \frac{(k-1)(j-1)}{n \mu}=\frac{1}{n} \frac{\left(\mathbb{E}\left[d^{2}\right]-\mathbb{E}[d]\right)^{2}}{(\mathbb{E}[d])^{3}}=\frac{\nu^{2}}{n \mu}
$$

Thus, if $\mathbb{E}\left[d^{2}\right]$ is finite, then the clustering coefficient vanishes in the thermodynamic limit.

\subsubsection{Power law random graphs}

The configuration model can used to generate graphs whose degree distribution follows a power law, i.e., $p_{k}=c k^{-\alpha} \forall k \geq d_{\text {min }}$, where $\alpha>1$, and $c$ is a normalization constant. Empirical studies of many real networks such as the internet [21], scientific collaboration networks [22], and collaboration network of movie actors [9] have reported a power law degree distribution motivating the study of power law random graphs [23].

If the exponent $\alpha \leq 1$, then the distribution cannot be normalized. When $\alpha>1$, it determines the finiteness of the moments of the degree distribution :

$$
\begin{array}{r}
\mu=\mathbb{E}[d]=c \sum_{k=d_{\min }}^{\infty} k^{-\alpha+1}<\infty \text { iff } \alpha>2 \\
\mathbb{E}\left[d^{2}\right]=c \sum_{k=d_{\min }}^{\infty} k^{-\alpha+2}<\infty \text { iff } \alpha>3 .
\end{array}
$$


Thus, the degrees of power law graphs with $\alpha \in(2,3)$ have a finite mean but an infinite variance, and are sometimes referred to as scale free networks as they lack a characteristic degree or scale. Another feature that makes the $\alpha \in(2,3)$ regime interesting is that the graphs are "ultra small world" by which we mean that they have a mean hop distance $h \sim \log \log n$ [24]; the diameter is also $\sim \log \log n$ if $d_{\min } \geq 3[25]$.

In order to estimate $d_{\max }$, from (1.8) we have

$$
\text { nc } \sum_{k=d_{\max }+1}^{\infty} k^{-\alpha} \approx 1
$$

Approximating the sum in (1.21) by an integral,

$$
d_{\max } \approx d_{\min } n^{1 /(\alpha-1)}
$$

This means that when $\alpha>1$, large power law graphs can have large degree vertices or hubs.

\subsection{Stochastic processes with random networks}

In this dissertation, we study stochastic processes involving random graphs. Specifically, our processes will be continuous time homogeneous Markov processes.

Let our initial random graph be $G_{0}$ whose distribution is known to be $\boldsymbol{\pi}_{0}$. We are interested in the distribution $\boldsymbol{\pi}_{t}(G)=\mathbb{P}\left(G_{t}=G\right)$ of the graph for $t>0$. Given the present state of the graph, the future states are independent of the past states. In an infinitesimal amount of time $\mathrm{d} t$, the distribution evolves according to

$$
\mathbb{P}\left(G_{t+\mathrm{d} t}=G \mid G_{t}=G^{\prime}\right)= \begin{cases}\Lambda\left(G^{\prime} \rightarrow G\right) \mathrm{d} t & \text { if } G \neq G^{\prime} \\ 1-\sum_{G^{\prime \prime} \neq G^{\prime}} \Lambda\left(G^{\prime} \rightarrow G^{\prime \prime}\right) \mathrm{d} t & \text { if } G=G^{\prime}\end{cases}
$$


where $\Lambda\left(G^{\prime} \rightarrow G\right)$ is the time independent transition rate from $G^{\prime}$ to $G$. This means

$$
\begin{aligned}
\dot{\pi}_{t}(G) & =\sum_{G^{\prime}}\left[\boldsymbol{\pi}_{t}\left(G^{\prime}\right) \Lambda\left(G^{\prime} \rightarrow G\right)-\boldsymbol{\pi}_{t}(G) \Lambda\left(G \rightarrow G^{\prime}\right)\right] \\
& =\sum_{G^{\prime}} \boldsymbol{\pi}_{t}\left(G^{\prime}\right)\left[\Lambda\left(G^{\prime} \rightarrow G\right)-\mathbb{1}_{G^{\prime}=G} \sum_{G^{\prime \prime}} \Lambda\left(G \rightarrow G^{\prime \prime}\right)\right] .
\end{aligned}
$$

Equation (1.24) may be written compactly in matrix form as

$$
\dot{\boldsymbol{\pi}}_{t}=\boldsymbol{\pi}_{t} \mathbf{R} \Rightarrow \boldsymbol{\pi}_{t}=\boldsymbol{\pi}_{0} e^{\mathbf{R} t}
$$

where $\boldsymbol{\pi}_{t}$ is the vector whose elements are $\boldsymbol{\pi}_{t}(G)$ and $\mathbf{R}$, called the generator of the stochastic process, is the matrix with elements

$$
R\left(G^{\prime}, G\right)=\Lambda\left(G^{\prime} \rightarrow G\right)-\mathbb{1}_{G^{\prime}=G} \sum_{G^{\prime \prime}} \Lambda\left(G \rightarrow G^{\prime \prime}\right)
$$

We say that steady state has been reached when $\dot{\boldsymbol{\pi}}_{t}=0$. This means that the steady state distribution $\boldsymbol{\pi}_{*}$ satisfies

$$
\boldsymbol{\pi}_{*} \mathbf{R}=0
$$

Equivalently, the steady state is given by

$$
\boldsymbol{\pi}_{*}=\lim _{t \rightarrow \infty} \boldsymbol{\pi}_{t}=\lim _{t \rightarrow \infty} \boldsymbol{\pi}_{0} e^{\mathbf{R} t}
$$

The steady state is an equilibrium if the more restrictive detailed balance condition is satisfied :

$$
\boldsymbol{\pi}_{*}\left(G^{\prime}\right) \Lambda\left(G^{\prime} \rightarrow G\right)=\boldsymbol{\pi}_{*}(G) \Lambda\left(G \rightarrow G^{\prime}\right) \quad \forall G, G^{\prime}
$$

so that the system looks the same going forward or backward in time. Systems that do not satisfy detailed balance are termed non-equilibrium systems. One is usually interested in the steady state value $\rho_{*} \equiv \rho\left(\boldsymbol{\pi}_{*}\right)$ of some attribute $\rho$ of the random graph. 


\subsection{Phase transitions involving networks}

The $\mathcal{G}(n, p)$ model discussed in Section 1.4.3 may be viewed as a growth model, where starting with $n$ vertices, one visits vertex pairs sequentially and adds an edge between them with probability $p$. The resulting graph in the $n \rightarrow \infty$ limit can then be treated as a thermodynamic system described by the parameter $\mu$. Fig. 1.1 shows that this system undergoes a phase transition when the parameter $\mu$ crosses 1 . In this Section, we discuss this phenomenon as a general feature exhibited by many systems.

A phase transition is a qualitative change in the nature of a thermodynamic system at a particular value $\lambda_{c}$, called the critical point, of the parameter $\lambda$ (e.g.: temperature) that describes the system. Note that $\lambda$ here could represent a collection of parameters. The "orderedness" or symmetry of the system is captured by some judiciously chosen attribute (typically a scalar, but not necessarily so) called its order parameter $\rho_{*}(\lambda)$. At the critical point, the function $\rho_{*}(\lambda)$ is non-analytic, and usually takes different functional forms on either side of $\lambda_{c}$. For instance, in the $\mathcal{G}(n, p)$ model, $\rho_{*}(\mu)=0$ below the critical point, and $\rho_{*}(\mu)=1+\mu^{-1} W\left(-\mu e^{-\mu}\right)$ above it. Thus, the phases of a system may be distinguished by their respective functional forms of $\rho_{*}(\lambda)$. Phases on either side of the critical point are sometimes called the ordered (lower symmetry) and disordered (higher symmetry) phases respectively. Depending on how the order parameter changes at the critical point, the phase transition is classified as continuous or discontinuous. The boiling of water to vapor at a fixed pressure (less than $217.7 \mathrm{~atm}$ ) is a discontinuous phase transition, while the transition of the Ising ferromagnet from a non-magnetic to a magnetic phase when cooled (at zero external field) is a continuous phase transition. Returning to the $\mathcal{G}(n, p)$ model, the order parameter is the fraction of vertices in the giant component, and the phase transition is continuous. 
Diverse physical systems have been found to show similar behavior near the critical region leading to the notion of universality, and attempts to classify systems into universality classes [26]. In the case of continuous transitions, many quantities (e.g.: order parameter, susceptibility $\partial \rho / \partial \lambda$, correlation length, etc.) associated with the system scale as a power law near the critical point, e.g.: $\left|\rho_{*}(\lambda)-\rho_{*}\left(\lambda_{c}\right)\right| \sim\left|\lambda-\lambda_{c}\right|^{-b}$. The scaling exponents depends only on a few aspects of the system such as its dimension, and not on its microscopic details, and thereby unify disparate systems into a small number of universality classes. We shall not dwell on this topic further, but instead direct the interested reader to classic books on the subject such as [27] and [28]. A detailed discussion, specific to networks, can be found in [29].

\subsubsection{Equilibrium vs. non-equilibrium systems}

In Section 1.5, we introduced equilibrium and non-equilibrium systems. In the context of phase transitions, it is instructive to elaborate on the differences between the two.

In an equilibrium system (or set of graphs) $\mathcal{G}$, one can define a Hamiltonian or "energy function" $H(G) \forall G \in \mathcal{G}$ such that the equilibrium distribution is the Gibbs distribution $\boldsymbol{\pi}_{*}(G) \sim e^{-\beta H(G)}$, where $\beta$ is some "inverse-temperature" parameter. Alternatively, the equilibrium distribution is the one that maximizes the entropy $-\sum_{G} \pi(G) \log \pi(G)$ [30]. This means that in (1.27), one already knows $\boldsymbol{\pi}_{*}$ up to a normalization constant (whose calculation, albeit, is usually challenging). If one needs to simulate the system to calculate thermodynamic quantities, one just needs to devise transition rates that satisfy detailed balance. As far as the equilibrium state is concerned, the dynamics is irrelevant. On the contrary, for non-equilibrium systems, $\boldsymbol{\pi}_{*}$ is not available for "free"; the dynamics are important making the analysis more involved [31]. Obvious examples of non-equilibrium systems are the ones that contain an absorbing state, i.e., a state that can be reached but not left. 
The systems studies in Chapters 2 and 3 are non-equibrium systems, while Chapter 4 deals with one in equilibrium.

\subsubsection{Approximate analytical methods}

The definition of phase transitions we gave earlier is reminiscent of the phenomenon of bifurcations in nonlinear dynamical systems. How are the two related? To start with, bifurcations are defined for finite dimensional dynamical systems, where as phase transitions occur in the thermodynamic limit, i.e., the phase space of the system is infinite dimensional. In finite dimensional stochastic systems, the transitions are not sharp due to the noise [32].

In almost all models of network dynamics, it is impossible to analytically calculate the steady state distribution $\boldsymbol{\pi}_{*}$. Even if the distribution is known, the calculation of $\rho_{*}$ and other statistics (expected value of properties of the graph) of interest is usually intractable. One therefore resorts to approximate methods. The primary aim of all these methods is to convert the infinite dimensional dynamical system into a finite dimensional one, which one can then describe and analyze using tools from nonlinear dynamics. The approximated nonlinear dynamical system might show bifurcations, which (hopefully) correspond to the phase transitions in the thermodynamic system, thus providing a rough qualitative picture of the transition.

For dynamics on networks, one usually attempts an analytical study by writing down the dynamical equations for the various moments of the network. By moment of a graph, we mean the expected number of copies a certain small labeled graph it contains (e.g.: two connected vertices, one in "on" state and the other "off"). We will call the number of vertices in the subgraph as the order of the moment. However, the equations for moments of a certain order will almost inevitably involve moments of higher orders, thereby forming an infinite hierarchy of equations. We can trucate this infinite set of equations at some level by what are known as mo- 
ment closure techniques [33]. Specifically, we approximate the higher order moments by lower order moments, and thereby create a closed set of equations. Obviously, higher the truncation level, the better the approximation. The simplest of these, i.e., truncating at the first level, is known as the mean field approximation (MFA). The MFA essentially replaces the complex interaction of the elements the graph among each other, by interactions of each element with an unknown average "field" that permeates the graph. Improving on the MFA is the pair approximation where one decomposes third order moments into products of second order ones [34].

\subsection{Overview of the projects}

This dissertation is a study of three models of network dynamics that form chapters 2, 3 and 4 respectively. Below we provide an overview of the projects.

Chapter 2 deals with a model, for the spread of opinion in a social network, known as the voter model. In this model each vertex of the network can be in one of many (2 in our case, which we label as 0 and 1) possible states (opinions). The network gets continuously updated by a randomly selected vertex adopting the opinion of one of its neighbors. Such a rule embodies the tendency of vertices to imitate their neighbors [35]. Our project titled "Graph Fission in an Evolving Voter Model", is a coevolving variant of the voter model wherein vertices are allowed to either adopt the neighbor's opinion with probability $1-\alpha$ or to "rewire" to another vertex in the network with probability $\alpha$. We study two versions here: rewire-to-same, where the rewiring happens only to a vertex of the same opinion and rewire-to-random, where it can happen to any randomly chosen vertex . Through simulations starting with various fractions $\rho_{0}$ of ' 1 ' vertices, we find a phase transition in the final minority fraction $\rho$ of voters as $\alpha$ is varied - continuous for the rewire-to-random case (Fig. 2.2) and discontinuous for the rewire-to-same case (Fig. 2.1). Specifically, in the rewireto-same case, there is a critical value $\alpha_{c}$ which does not depend on $\rho_{0}$, with $\rho \approx \rho_{0}$ 
for $\alpha>\alpha_{c}$ and $\rho \approx 0$ for $\alpha<\alpha_{c}$. In the rewire-to-random case, the transition point $\alpha_{c}\left(\rho_{0}\right)$ depends on the initial density $\rho_{0}$. For $\alpha>\alpha_{c}\left(\rho_{0}\right), \rho \approx \rho_{0}$, but for $\alpha<\alpha_{c}\left(\rho_{0}\right)$ we have $\rho\left(\alpha, \rho_{0}\right)=\rho(\alpha, 1 / 2)$. The main result of the project is an explanation of these phase transitions.

In the simulations, we notice that for the rewire-to-random version, the number of discordant edges $n_{01}$ quickly drops to about half of its initial value and that there is a significant correlation between the number $n_{1}$ of vertices in state 1 and $n_{01}$ (Fig. 2.3). Further, a plot of $n_{01}$ vs. $n_{1}$ reveals an interesting curve which we call the arch (Fig. 2.4). Guided by these observations, we conjectured that, after an initial transient period, all statistics on the network converge to the arch, i.e., depends only on $n_{1}$ and diffuses on it until $n_{01}$ becomes zero. The arch is able to explain the continuous phase transition as follows. The support interval of the arch shrinks with increasing $\alpha$. So $n_{1}$ values that start outside this interval remain unaltered at equilibrium, whereas $n_{1}$ values that start within the support interval end up at either boundaries of the interval. For the rewire-to-same model, the arches always span $(0,1)$ but flatten with increasing $\alpha$, coinciding with the $n_{1}$ axes at the critical value and inverting beyond that. This explains the discontinuous phase transition. We also try to determine the equation for the arch through approximate calculations - mean field, pair approximation and approximate master equation, whose agreement with the simulation results improve in that order.

The author was one among eight researchers who participated in this project that started in the fall of 2010. The author's own association with the project began in the summer of 2011 and his work involved performing some of the approximate calculations and numerical simulations, in particular, Fig. 2.3which led to the conjecture about the arch. The contents of Chapter 2 are taken from the published version of the work [36].

Chapter 3 deals with the quadratic contact process (QCP) on random graphs 
and their associated phase transitions. The quadratic contact process (QCP) is a natural extension of the well studied linear contact process. In the linear contact process, similar to the voter model, vertices can be in one of two states - infected (1) or susceptible (0); and infected individuals infect susceptible neighbors at rate $\lambda$ and at rate 1 , recover $(1 \longrightarrow 0)]$. In the QCP, a combination of two 1 's is required to effect a $0 \longrightarrow 1$ change. We extend the study of the QCP, which so far has been limited to lattices, to complex networks. However, unlike in the evolving voter model, the topology of the network does not change. We define two versions of the QCP - vertex centered (VQCP) and edge centered (EQCP) with birth events $1-0-1 \longrightarrow 1-1-1$ and $1-1-0 \longrightarrow 1-1-1$ respectively, where '-' represents an edge. We investigate the effects of network topology by considering the QCP on random regular, Erdős-Rényi and power law random graphs. We perform two types of mean field calculations suited for networks with homogeneous and with heavy tailed degree distributions respectively, as well as simulations to find the steady state fraction of occupied vertices as a function of the birth rate. We also give a few rigorous results about the models. Combining our simulation, mean field calculation and rigorous results, we conclude that on the homogeneous graphs - random regular and Erdős-Rényi graphs, there is a discontinuous phase transition with a region of bistability, whereas on the heavy tailed power law graph, the transition is continuous. Furthermore, the critical birth rate is positive in the former but zero in the latter.

Chapter 4 introduces a class of models of evolving spatial networks which we call the Evolving Spatial Network Model (ESNM), motivated by earlier models studied by Henry et al. [37] and by Magura et al. [38] in the context of social networks. The network evolves with a tendency to reduce its total length by rewiring of edges only, following a Metropolis-Hastings algorithm. In a rewiring attempt, shorter edges are always accepted while longer edges are accepted with a probability that decays exponentially with the change in length. In a second version of the model, we also 
require the network to satisfy some constraint (for e.g., the network is connected) at all times. The evolving networks have an equilibrium distribution $\boldsymbol{\pi}(G) \sim \exp [-\beta \times$ total edge length] which depends on four parameters - the dimension of the space, the average degree, $\beta$, and the constraint.

We first consider the ESNM with no constraint imposed on the networks, which could be a model for a social network where individuals have fixed opinions and adjust their ties to be connected to people with similar opinion. The extreme values of the parameter $\beta$ lead to equilibrium networks that have been well studied in the literature: $\beta=0$ corresponds to the Erdős-Rényi random graph, while $\beta \rightarrow \infty$ results in the random geometric graph where every vertex is connected to all its spatial neighbors up to a certain fixed distance. For intermediate values of $\beta$, following Magura et al. , we use an equivalent percolation model wherein every vertex pair $\{x, y\}$ is independently connected with a probability $g(|x-y|)$. A proper choice of the function $g(\cdot)$ makes the properties of networks in both models approach each other. Using the percolation model, we compute analytically and by simulation some quantities of interest, such the distribution of edge lengths, the fraction of vertices in the largest component, and the clustering coefficient.

We also study the ESNM with the constraint that the network be connected. This evolution model has potential applications in the design of transportation and distribution networks. With this constraint and $\beta$ large, the model can be viewed as an algorithm to find the shortest length connected network (which we refer to as the optimized network) with a given mean degree over a set of points. On the other hand, with $\beta=0$, the equilibrium network is a random connected network $(\mathrm{RCN})$. The connectedness constraint precludes an equivalent percolation version, as it existed for the unconstrained model. So we obtain an approximation to the optimized network by simulating the model with a large value of $\beta$.

The projects in Chapters 3 and 4 are joint works of the author with his advisor 
Rick Durrett. The author was involved in both the computational and analytical aspects of the research. The contents of Chapter 3 is taken from the published version [39] of the work, while that of Chapter 4 is from the manuscript that will be submitted soon after his thesis defense.

In Chapter 5, we provide some closing remarks on the results from the three projects. We conclude this introductory chapter by pointing out the key similarities and differences between the stochastic network dynamics in the models considered in the three projects : In all the cases, the starting network is random in its vertex set, its edge set, and in its vertex states. In the QCP and ESNM, the evolution happens exclusively in their vertex states and edge sets respectively. Thus the QCP is a dynamics on networks, while the ESNM deals with dynamics of networks. In contrast, the evolution of the edge set and the vertex states are coupled in the evolving voter model, making it a coevolving network model. Furthermore, the ESNM is an equilibrium model, while the evolving voter model and the QCP are non-equilibrium models. 


\section{Graph Fission in an Evolving Voter model}

In recent years, research efforts from different disciplines have combined with established studies in social network analysis and random graph models to fundamentally change the way we think about networks. Significant attention has focused on the implications of dynamics in establishing network structure, including preferential attachment, rewiring, and other mechanisms [40-44]. At the same time, the impact of structural properties on dynamics on those networks has been studied, [45], including the spread of epidemics [1,46-48], opinions [11,35, 49], information cascades [50-52], and evolutionary games $[53,54]$. Of course, in many real-world networks the evolution of the edges in the network is tied to the states of the vertices and vice versa. Networks that exhibit such a feedback are called adaptive or coevolutionary networks $[55,56]$. As in the case of static networks, significant attention has been paid to evolutionary games [57-60] and to the spread of epidemics [61-65] and opinions [66-71], including the polarization of a network of opinions into two groups $[37,72]$. In this paper, we examine two closely related variants of a simple, abstract model for coevolution of a network and the opinions of its members. 


\subsection{Holme-Newman Model}

Our starting point is the model of Holme and Newman [73-76]. They begin with a network of $n$ vertices and $m$ edges, where each vertex $x$ has an opinion $s(x)$ from a set $\mathcal{S}$ of possible opinions and the number of people per opinion $a_{n}=n /|\mathcal{S}|$ stays bounded as $n$ gets large. On each step of the process, a vertex $x$ is picked at random. If its degree $d(x)=0$, nothing happens. If $d(x)>0$, then (i) with probability $\alpha$ an edge attached to vertex $x$ is selected and the other end of that edge is moved to a vertex chosen at random from those with opinion $s(x)$; (ii) otherwise (i.e., with probability $1-\alpha)$ a random neighbor $y$ of $x$ is selected and we set $s(x)=s(y)$. This process continues until there are no longer any edges connecting individuals with different opinions.

When $\alpha=1$, only rewiring steps occur, so once all of the $m$ edges have been touched, the graph has been disconnected into $|\mathcal{S}|$ components, each consisting of individuals who share the same opinion. Since none of the opinions have changed the components are small (i.e., their sizes are Poisson with mean $a_{n}$ ). By classical results for the coupon collector's problem, this requires $\sim m \log m$ updates, (see e.g., page 57 in [77]).

In contrast, for $\alpha=0$ this system reduces to the voter model on a static graph. If we suppose that the initial graph is an Erdös-Rényi random graph in which each vertex has average degree $\mu>1$, then, as we discussed in Section 1.4.3, there is a giant component that contains a positive fraction, $b n$, of the vertices and the second largest component is small having only $\mathcal{O}(\log n)$ vertices, i.e., when $n$ is large the size will be $\approx c_{\mu} \log n$, where $c_{\mu}$ is a constant that depends on $\mu$ (see e.g., Chapter 2 of [11]). The voter model on the giant component will reach consensus in $\mathcal{O}\left(n^{2}\right)$ steps (see, e.g., Section 6.9 of [11]), so the end result is that one opinion has $b n$ followers while all of the other groups are small. 
Using simulation and finite size scaling, Holme and Newman showed that there is a critical value $\alpha_{c}$ so that for $\alpha>\alpha_{c}$ all of the opinions have a small number of followers at the end of the process, while for $\alpha<\alpha_{c}$ "a giant community of likeminded individuals forms." When the average degree $\mu=2 m / n=4$ and the number of individuals per opinion $a_{n} \rightarrow 10$, this transition occurs at $\alpha_{c} \approx 0.46$.

\subsection{Our Model \& Simulation Results}

The rewire to same model we study differs from that of Holme and Newman in two ways: (a) we consider two opinions (called 0 and 1) instead of a number proportional to the size of the graph; and (b) on each step, we pick a discordant edge $(x, y)$ at random rather than a vertex, avoiding the problem of picking vertices with degree 0 or vertices that agree with all of their neighbors. With probability $1-\alpha$ the voter at $x$ adopts the opinion of the voter at $y$. Otherwise (i.e., with probability $\alpha$ ), $x$ breaks its connection to $y$ and makes a new connection to a voter chosen at random from those that share its opinion. The process continues until there are no edges connecting voters that disagree.

Despite the differences in implementation, this rewire to same model has a phase transition similar to that of Holme and Newman. In particular, the final fraction $\rho$ of voters with the minority opinion undergoes a discontinuous transition at a value $\alpha_{c}$ that does not depend on the initial density. Figure 2.1 shows results of simulations for the rewire to random model starting from an initial graph that is Erdös-Rényi with $n=100,000$ vertices and average degree $\mu=4$. Opinions are initially assigned randomly with the probability of opinion 1 given by $\rho_{0}=0.5,0.25,0.1$, and 0.05 . The figure shows the final fraction $\rho$ of voters with the minority opinion from five realizations for each $\rho_{0}$. For $\alpha>\alpha_{c} \approx 0.43$ we observe $\rho \approx \rho_{0}$ and for $\alpha<\alpha_{c} \rho \approx 0$.

We also study a rewire to random variant of this model that differs from the rewire to same model in only one way: $x$ makes its new connection to a voter chosen 


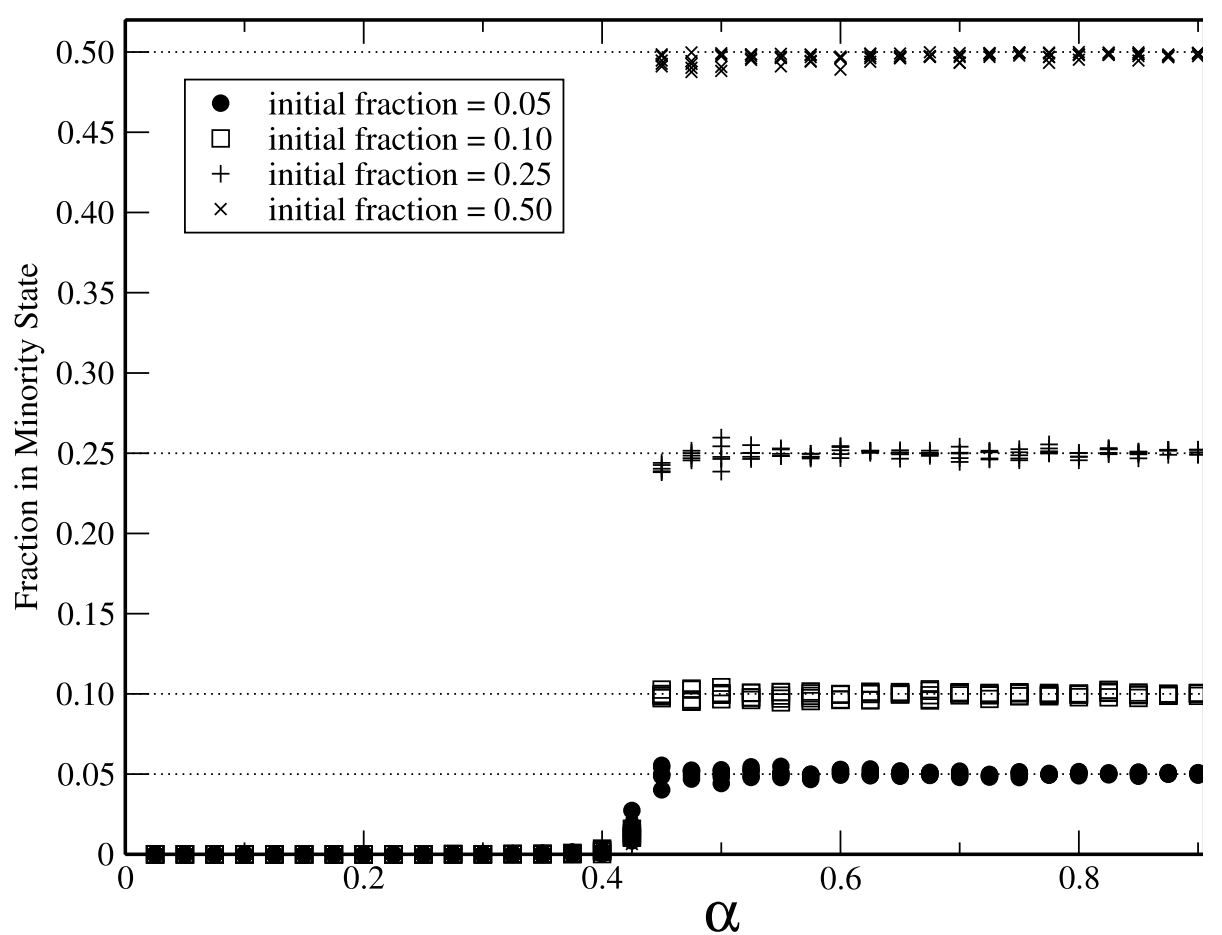

FiguRE 2.1: Simulation results for rewire to same model, starting from Erdös-Rényi graphs with $n=100,000$ nodes and average degree $\mu=4$.

at random from all of the vertices in the graph. This single difference leads to fundamentally different model outcomes, as seen in Figure 2.2, showing simulation results for the rewire to random model on initially Erdös-Rényi graphs with $n=$ 100,000 nodes and average degree $\mu=4$ for $\rho_{0}=0.5,0.25,0.1$, and 0.05 . When $\rho_{0}=0.5$ the fraction in the minority is constant at 0.5 over $\left[\alpha_{c}(0.5), 1\right]$ and then decreases continuously to a value near 0 as $\alpha$ decreases to 0 .

The behavior of our models for $\alpha>\alpha_{c}$ is easy to understand. As in the case of the Holme and Newman model, we expect consensus to be reached in $\mathcal{O}(n \log n)$ steps when $\alpha=1$ and in $\mathcal{O}\left(n^{2}\right)$ steps when $\alpha=0$. We define the boundary between the fast and slow consensus regimes to be the value of $\alpha$ where the average number of steps needed to reach consensus is $n^{3 / 2}$ (any power between 1 and 2 would give the same results when $n \rightarrow \infty)$. When an edge is chosen between voters with different opinions then a rewiring event does not change the number of 1's, while a voting 


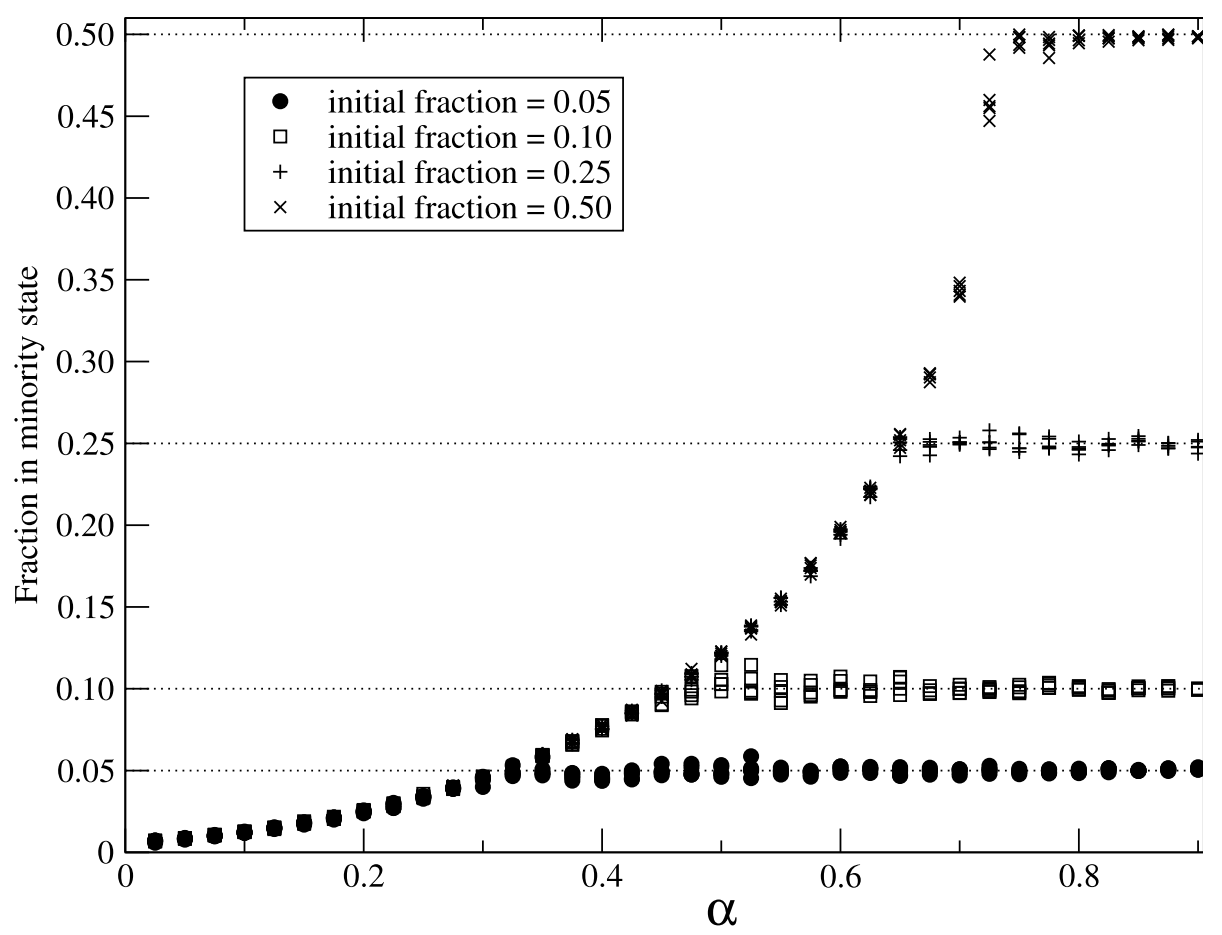

FiguRE 2.2: Simulation results for the rewire to random model, starting from ErdösRényi graphs with $n=100,000$ nodes and average degree $\mu=4$.

event will increase and decrease the number of 1's with equal probability, i.e., the number of 1's is a random walk that on each step stays constant with probability $\alpha$. The central limit theorem implies that when consensus is reached in $\mathcal{O}\left(n^{3 / 2}\right)$ steps the typical change in the number of 1's from the initial configuration is $\mathcal{O}\left(n^{3 / 4}\right)$. Hence, when the initial fractions of 1 's is $\rho_{0} \leq 1 / 2$ the final fraction $\rho$ with the minority opinion will be approximately equal to $\rho_{0}$.

Turning to the curves in Figure 2.2 for $\rho_{0}=0.25,0.1$ and 0.05 , we see that each initial density $\rho_{0}$ has a critical value $\alpha_{c}\left(\rho_{0}\right)$ so that for $\alpha>\alpha_{c}\left(\rho_{0}\right)$ we have $\rho\left(\alpha, \rho_{0}\right)=\rho_{0}$, while for $\alpha<\alpha_{c}\left(\rho_{0}\right)$ we have $\rho\left(\alpha, \rho_{0}\right)=\rho(\alpha, 0.5)$. Since all of the $\rho\left(\alpha, \rho_{0}\right)$ agree with $\rho(\alpha, 0.5)$ when they are $<\rho_{0}$, we call the graph of $\rho(\alpha, 0.5)$ on $\left[0, \alpha_{c}(0.5)\right]$ the universal curve. The main goal of this paper is to explain this phenomenon. 


\subsection{Quasi-stationary distributions}

Let $n_{i}$ be the number of vertices in state $i$. Our first clue to the reason for a universal curve in the rewire to random model came from Figure 2.3, which shows the change over time of the fraction of vertices with the minority opinion $\min \left\{n_{1}, n_{0}\right\} / n$ and the number of edges connecting vertices with opposite opinions, $n_{1-0}$, for a simulation in which the initial density of 1 's is $\rho_{0}=1 / 2, \alpha=0.3$, the number of nodes is $n=1000$, and we start with an Erdös-Rényi graph with average degree $\mu=4$. In the visualization of these results and the theoretical discussions that follow, the model is considered in continuous time with each edge subject to change at times of a rate one Poisson process. The sequence of states visited by the model is the same in discrete or continuous time, but $t m$ updates correspond to continuous time $t$. Hence, in the slow consensus regime $\mathcal{O}\left(n^{2}\right)$ updates becomes time $\mathcal{O}(n)$.

There are $m \approx 2000$ edges in this graph simulated in Figure 2.3, so the initial number of $1-0$ edges is $\approx 1000$, but the curve drops very quickly to a value near 600 , and then begins to change more slowly. The second key observation is that the number of 0-1 edges and the fraction with the minority opinion $\min \left\{n_{1}, n_{0}\right\} / n$ appear to be strongly correlated. The initial transient and the reason for the correlation will be seen more clearly in Figure 2.4.

To explain the key insight derived from this simulation, we recall results for the voter model on the $D$-dimensional integer lattice $\mathbb{Z}^{D}$ in which each vertex decides to change its opinion at rate 1 , and when it does, it adopts the opinion of one of its $2 D$ nearest neighbors chosen at random. Let $s_{t}(x)$ be the opinion of the voter at $x$ at time $t$. Holley and Liggett (see [78], [79]) proved the following result.

Theorem 1. In $D \leq 2$, the voter model approaches complete consensus that is, if $x \neq y$ then $\mathbb{P}\left(s_{t}(x) \neq s_{t}(y)\right) \rightarrow 0$. In $D \geq 3$ if the voter model starts from product measure with density $u$, i.e., $s_{0}^{(u)}(x)$ are independent and equal to 1 with probability 

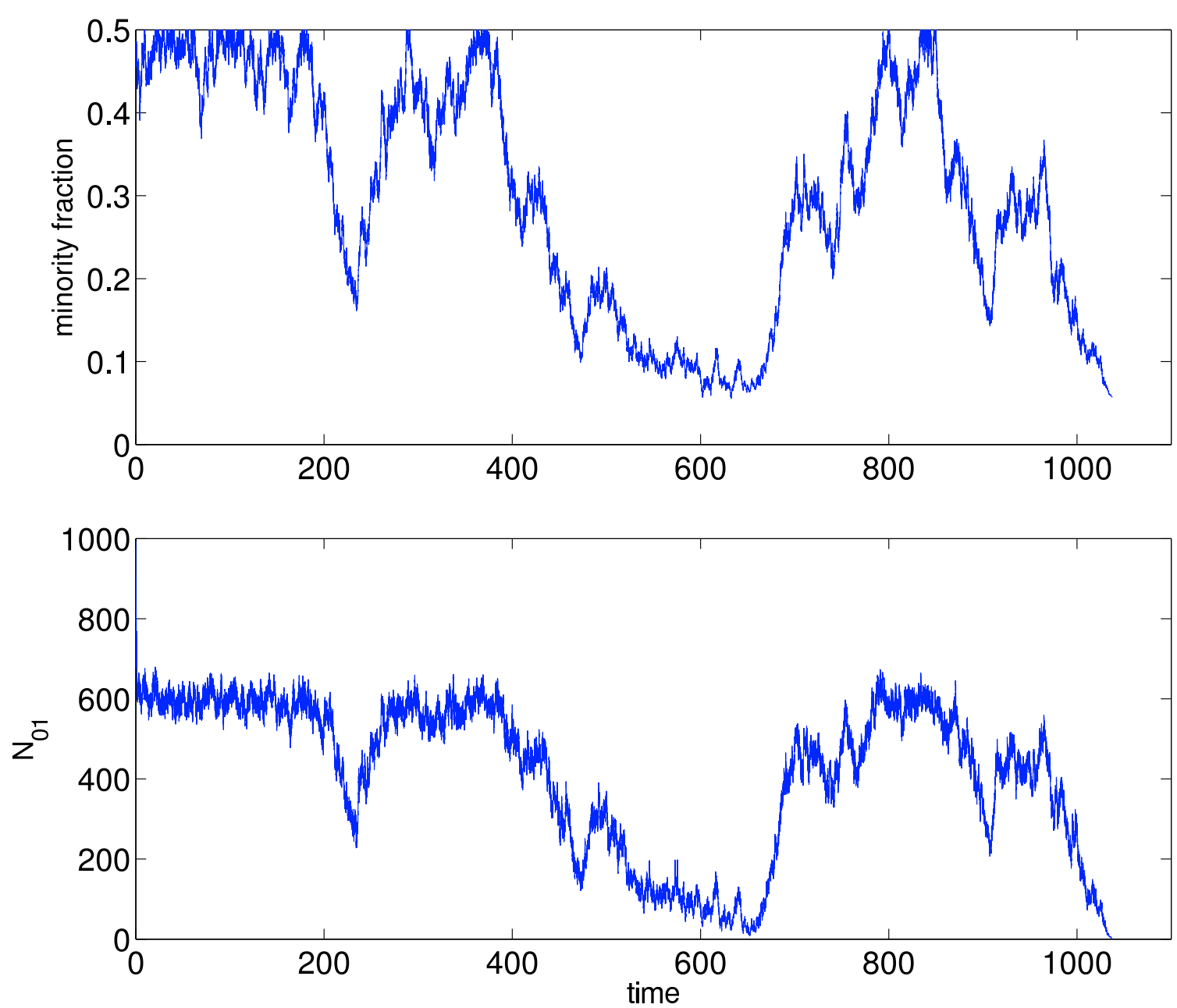

FiguRE 2.3: Fraction of nodes with the minority opinion $\left(\min \left\{n_{0}, n_{1}\right\} / n\right)$ and the number of discordant edges $n_{10}$ versus time, for a simulation of $n=1000$ nodes, $\rho_{0}=0.5$, and $\alpha=0.3$.

$u$ then $s_{t}^{(u)}$ converges in distribution to a limit $\nu_{u}$, which is a stationary distribution for the voter model.

Simulations of the voter model are done on a finite set, typically the torus $(\mathbb{Z} \bmod$ $L)^{D}$. In this setting the behavior of the voter model is "trivial" because it is a finite Markov chain with two absorbing states, all 1's and all 0's. As the next result due to Cox and Greven [80] shows, the voter model has interesting behavior along the road to absorption.

Theorem 2. If the voter model on the torus in $D \geq 3$ starts from product measure 


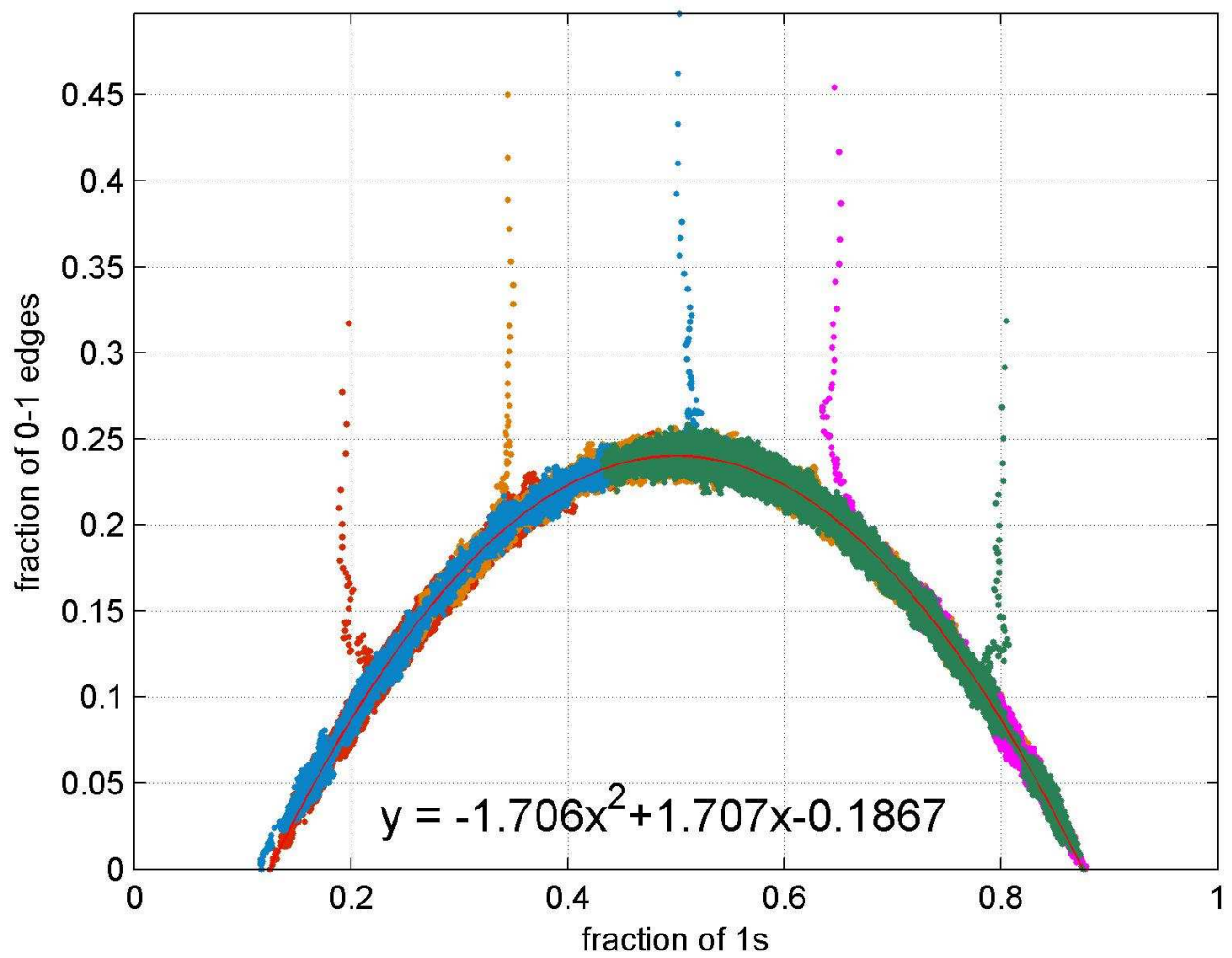

Figure 2.4: Plot of $n_{10} / m$ versus $n_{1} / n$ when $\alpha=0.5$ in the rewire to random case. Five simulations starting from $\rho_{0}=0.2,0.35,0.5,0.65$, and 0.8 are plotted in different colors. These results are from graphs with $n=10,000$ vertices and plotted every 1,000 steps.

with density $u$ then at time $n t$ it looks locally like $\nu_{\theta(t)}$ where the density $\theta_{t}$ changes according to the Wright-Fisher diffusion process

$$
d \theta_{t}=\sqrt{\eta_{D} \cdot 2 \theta_{t}\left(1-\theta_{t}\right)} d B_{t}
$$

and $\eta_{D}$ is the probability that two random walks starting from neighboring sites never hit, and $B_{t}$ is standard Brownian motion.

In the next section we will describe conjectures for the evolving voter model that are analogues of the the last theorem. To prepare for stating our conjectures note that (i) while the voter model on the torus does not have a nontrivial stationary 
distribution, it does have a one parameter family of quasi-stationary distributions ${ }^{1}$ that look locally like $\nu_{u}$ and (ii) the quantity under the square root in the WrightFisher diffusion is, by results of Holley and Liggett, the expected value of $n_{10} / m$ under $\nu_{\theta(t)}$.

\subsection{Conjectures}

Our next goal is to use simulation results to formulate the analogues of the Cox and Greven result for our two evolving voter models, beginning with the more interesting rewire to random case. Figure 2.4 shows results from simulations of the system with $\alpha=0.5$. The initial graph is Erdös-Rényi with $n=10,000$ vertices and average degree $\mu=4$. Observations of the pair $\left(n_{1} / n, n_{10} / m\right)$ are plotted every 1,000 steps starting from densities $\rho_{0}=0.2,0.35,0.5,0.65$ and 0.8 . The plotted points converge quickly to a curve that is approximately (fitting to a parabola) $1.707 x(1-x)-0.1867$ and then diffuse along the curve until they hit the axis near 0.125 or 0.875 . Thus the final fraction with the minority opinion $\rho \approx 0.125$, a value that agrees with the universal curve in Figure 2.2 at $\alpha=0.5$.

The fact that, after the initial transient, $n_{10} / m$ is a function of $n_{1} / n$ supports the conjecture that the evolving voter model has a one parameter family of quasistationary distributions, for if this is true then the values of all of the graph statistics can be computed from $n_{1} / n$. To further test this conjecture, we examined the joint distribution of the opinions at three sites. Let $n_{i j k}$ be the number of oriented triples $x-y-z$ of adjacent sites having states $i, j, k$ respectively. Note for example, that in the 010 case this will count all such triples twice.

Figure 2.5 shows a plot of $n_{010} / n$ versus $n_{1} / n$. After an initial transient, the observed values stay close to a curve that is well approximated by a cubic. Simula-

${ }^{1}$ Quasi-stationary distribution is the eigenvector associated with the smallest non-zero eigenvalue of the rate matrix. It gives the distribution of states of the network, given that it has not reached the absorbing state $n_{10}=0$. 


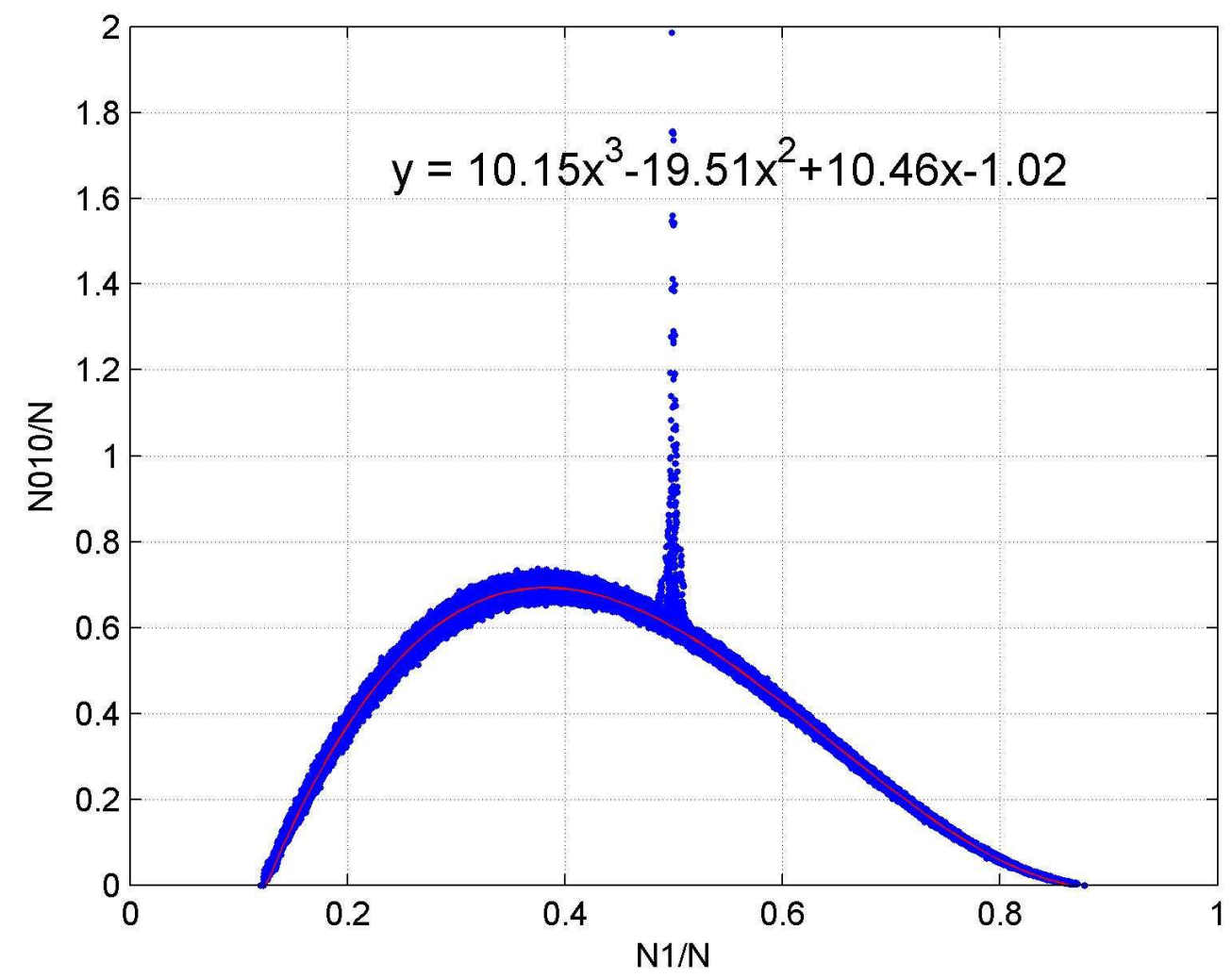

FiguRE 2.5: Plot of $n_{010} / n$ versus $n_{1} / n$ when $\alpha=0.5$ in the rewire to random case. All simulations start at $\rho_{0}=0.5$, since multiple runs from one starting point are enough to explore all of the arch. These results are from graphs with $n=100,000$ vertices and plotted every 10,000 steps.

tions of the other $n_{i j k}$ show similar behavior. Since the numbers of 010 triples must vanish when the number of 1-0 edges do, the fitted cubic shares two roots with the quadratic approximating the graph of $n_{10} / m$ versus $n_{1} / n$. This quadratic curve (see again Figure 2.4 for $\alpha=0.5$ ) is fundamental to our understanding of the observed system behavior, and we hereafter refer to it as the arch.

The phenomena just described for $\alpha=0.5$ also hold for other values of $\alpha$. Figure 2.6 shows the arches that correspond to $\alpha=0.1,0.2, \ldots, 0.7$. Numerical results show that the curves are well approximated by $c_{\alpha} \rho_{0}\left(1-\rho_{0}\right)-b_{\alpha}$. Let $(v(\alpha), 1-v(\alpha))$ be the support interval where the arch has positive values. Simulations show that if 


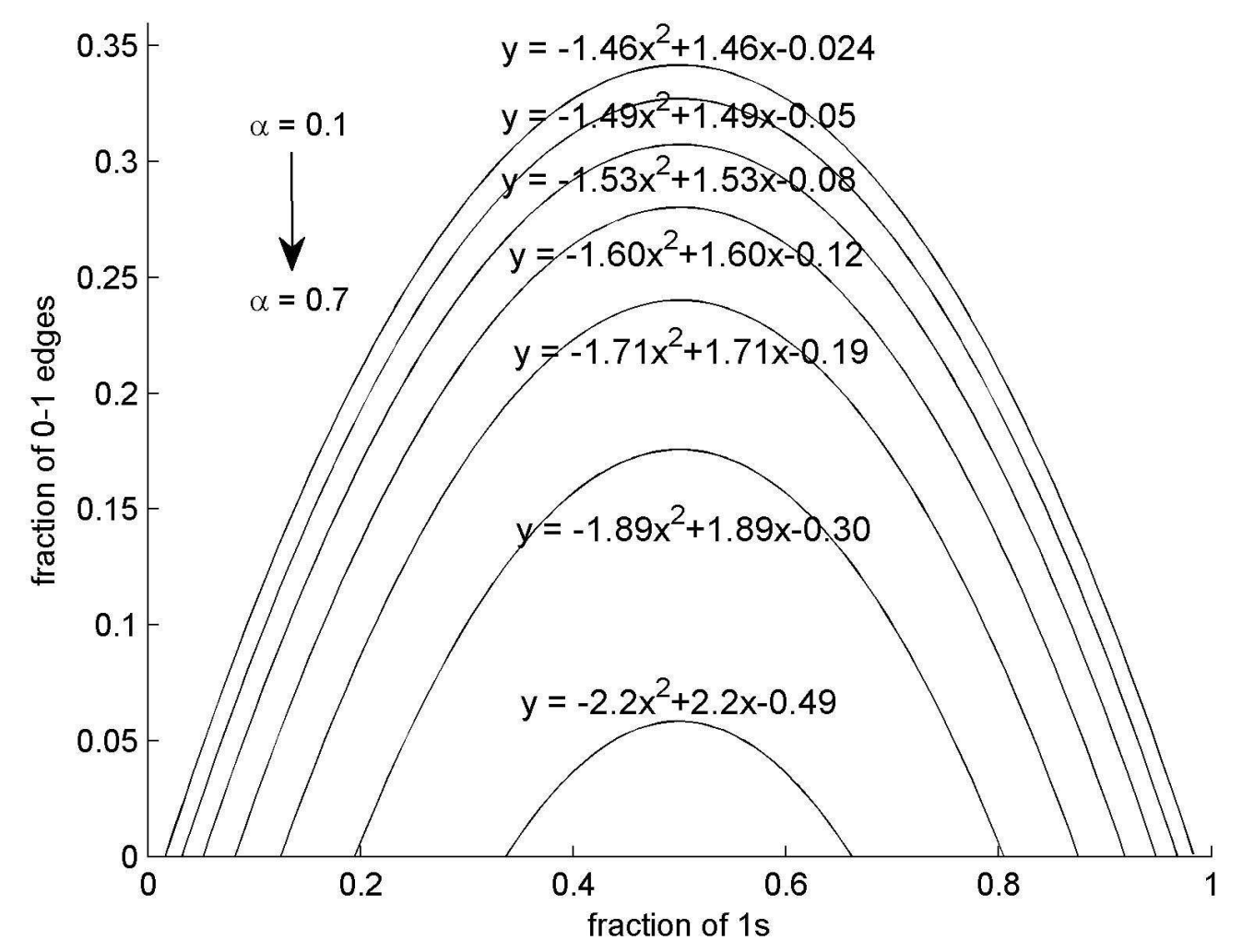

FiguRE 2.6: Observed arches for the rewire to random model. The specified parabolas are fits to simulation data with $n=10,000, \mu=4$.

$\rho_{0}<v(\alpha)$ then the simulated curve rapidly goes almost straight down and hits the axis where $n_{10}=0$.

Conjecture 1. In the rewire to random model if $\alpha<\alpha_{c}(1 / 2)$ and $v(\alpha)<\rho_{0} \leq 1 / 2$ then starting from product measure with density $\rho_{0}$ of 1's, the evolving voter model converges rapidly to a quasi-stationary distribution $\nu_{\alpha, \rho_{0}}$. At time th the evolving voter model looks locally like $\nu_{\alpha, \theta(t)}$ where the density changes according to a generalized Wright-Fisher diffusion process

$$
d \theta_{t}=\sqrt{(1-\alpha)\left[c_{\alpha} \theta_{t}\left(1-\theta_{t}\right)-b_{\alpha}\right]} d B_{t}
$$

until $\theta_{t}$ reaches $v(\alpha)$ or $1-v(\alpha)$. 


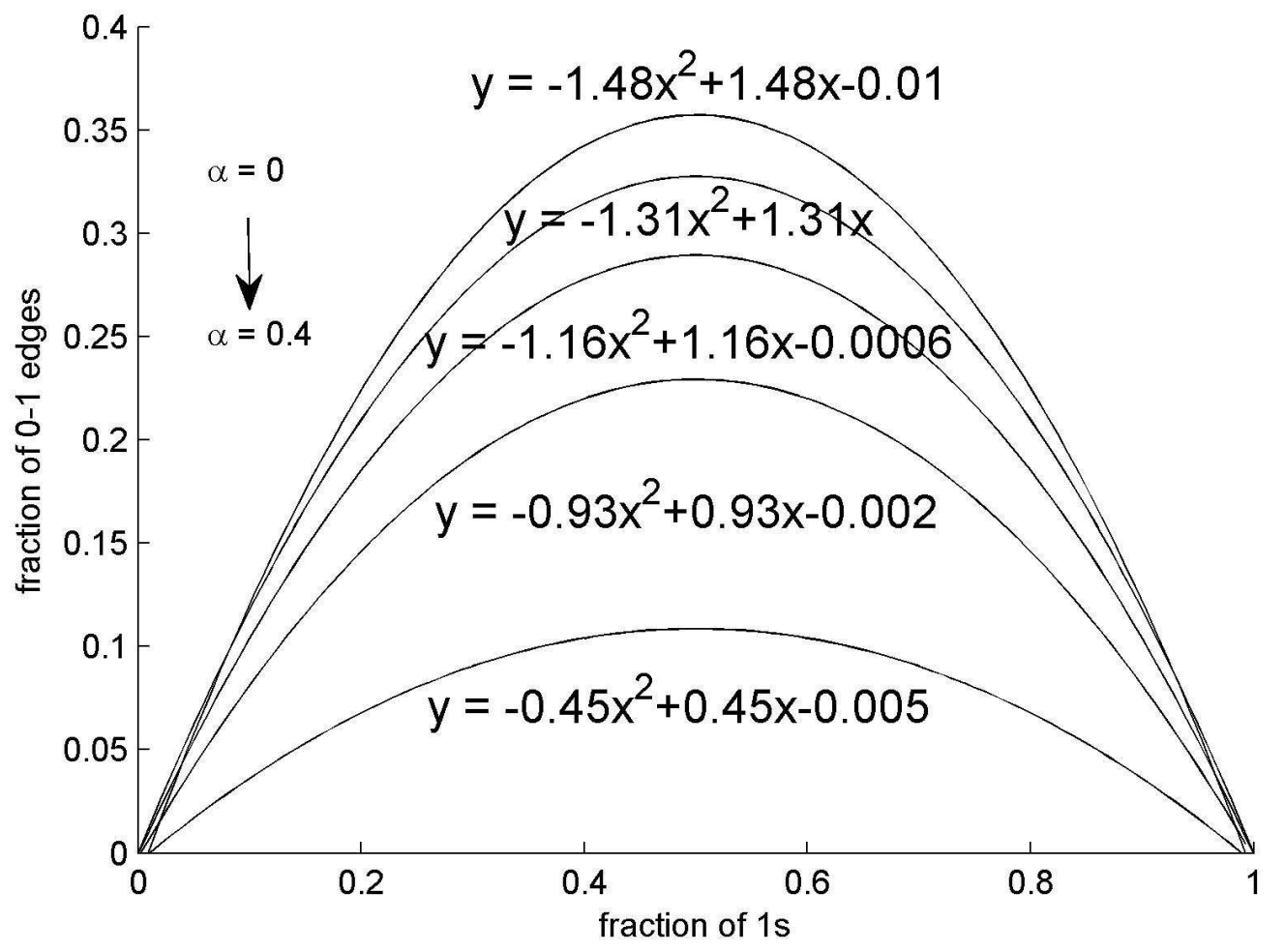

FiguRE 2.7: Observed arches for rewire to same model. The specified parabolas are fits to simulation data with $n=10,000, \mu=4$.

Here the quantity under the square root is $(1-\alpha) n_{10} / m$ with $(1-\alpha)=$ the fraction of steps that are voter steps, since rewiring steps do not change the number of 1's.

If Conjecture 1 is true then the universal curve in Figure 2.2 has $\rho(\alpha, 0.5)=v(\alpha)$ for $\alpha<\alpha_{c}(0.5)$. When $\alpha$ is close to $\alpha_{c}(0.5), v(\alpha) \approx 1 / 2$, so when the evolving voter model hits $n_{10}=0$ both opinions are held by large groups, and the graph splits into two giant connected components (that is, their size is proportional to $n$ for large graphs).

Though the nature of the phase transition looks different in the rewire to same model, the underlying picture is the same. Figure 2.7 shows arches computed from simulations for the rewire to same model that correspond to the ones in Figure 
2.6 for the rewire to random model. However, now all the arches have the same support interval, $(0,1)$, and the formulas in that figure show that the curves are well approximated by $c_{\alpha} \rho_{0}\left(1-\rho_{0}\right)$ for different values of $c_{\alpha}$.

In the rewire to same case, Vazquez, Eguíluz and San Miguel [75] were the first to notice that the fraction of active links $n_{10} / m$ plotted versus the fraction of 1 's converged rapidly to an arch and then diffused along it (see their Figure 4). However, they did not formulate the following:

Conjecture 2. In the rewire to same model the behavior is as described in Conjecture 1 but now $b_{\alpha}=0$, so $\alpha_{c}$ is independent of the initial density $\rho_{0}$, and for $\alpha<\alpha_{c}$, $\rho \approx 0$.

\subsection{Discussion}

We have considered a model in which the opinions of individuals and network structure coevolve. Based on the simulations we conclude that,

(i) there is a discontinuous transition in the rewire to same model, similar to that in Holme and Newman [73], which occurs at an $\alpha_{c}$ independent of the initial fraction $\rho_{0}$ of 1's;

(ii) there is a continuous transition in the rewire to random model at the critical value $\alpha_{c}\left(\rho_{0}\right)$ that depends on $\rho_{0}$, and the curves for the final fraction $\rho\left(\alpha, \rho_{0}\right)$ of voters in the minority agree with $\rho(\alpha, 1 / 2)$ for $\alpha<\alpha_{c}\left(\rho_{0}\right)$.

Thus our study shows how the disintegration of a social network containing two opinions on a polarizing issue, could depend on the strength of the polarization (i.e, the parameter $\alpha$ ), and on whether the individuals have prior knowledge of the opinion of strangers whom they might befriend. Although the latter appears to be a minor variation in the dynamics of the model, it results in a large change in the qualitative behavior, i.e., discontinuous vs. continuous transition. If we compare 
Fig. 2.1and Fig. 2.2, the behavior in the region between $\alpha \approx 0.43$ and $\alpha \approx 0.74$, is not surprising. That is, for a given rewiring probability, if voters always try to connect to someone of the same opinion, the network splits very quickly, giving them insufficient time to influence each other. On the other hand, if the voters tend to "befriend strangers", the network is unlikely to break for a long time during which voters alter each others' opinions. However, when the rewiring probability is outside the above mentioned range, the network stays intact for the same $(\alpha>0.74)$, or even a longer $(\alpha<0.43)$, period of time when voters are specifically choosing like minded friends, rather than any stranger. This is a bit counterintuitive. 


\section{3}

\section{The Quadratic Contact Process on Complex Networks}

\subsection{Introduction}

Inspired by technological and social networks, the study of complex networks has seen a surge in the past fifteen years $[1,10,43,44,81]$. Research has traditionally progressed in two distinct directions - dynamics of networks and dynamics on networks. The former is concerned with the formation of a network or change in its structure with time, whereas the latter deals with processes (deterministic or stochastic) taking place on a fixed network. Preferential attachment and its many generalizations [40, $82]$ are prototypical examples of the first type. Examples of the second are epidemics $[46,47,83,84]$, the voter model for the spread of an opinion [11,35,49], cascades [50-52] that model spread of a technology, and evolutionary games [53]. The phase transitions $[29,45]$ associated with these models have been of particular interest.

In the mathematics community, spatial models are studied under the heading of interacting particle systems [85]. One of the simplest models of those models is the contact process [86-88] (equivalent to the SIS model in epidemiology). In the 
linear contact process each site can be in one of two states which we will call 1 and 0. 0's become 1 at a rate proportional to the number of 1 neighbors they have and 1's become 0 at a constant rate (here and in all following models, unless otherwise specified, the processes occur in continuous time).

A natural extension of the linear process is the quadratic contact process (QCP) where each $0 \rightarrow 1$ event will require two other sites in state 1 . We will occasionally refer to 1 as being the "occupied" state and 0 as being "vacant", and the events $0 \rightarrow 1$ and $1 \rightarrow 0$ to be birth and death events respectively. At this stage, the model is quite general in that we do not specify where the two 1 's that cause the $0 \rightarrow 1$ event must be located with respect to the 0 . On the $2 \mathrm{D}$ lattice, specifying these locations leads to different realizations of the QCP. For example, Toom's North-East-Center model (originally defined in discrete time) allows a 0 at site $x$ to be filled if its neighbors $x+(0,1)$ and $x+(1,0)$ are occupied [89]. Chen [90,91] has studied versions of Toom's model in which two or three specified adjacent pairs or all four adjacent pairs are allowed to reproduce. Evans, Guo and Liu [92-96] have studied the QCP as a model for adsorption-desorption on a two dimensional square lattice. In the version of the model studied by Liu [96], 0 becomes 1 at rate proportion to the number of adjacent pairs of 1 neighbors. He found a discontinuous phase transition with a region of bistability, where the 1's die out starting from a small density. He also found that by introducing spontaneous births at a sufficiently high rate, the transition becomes continuous.

The QCP is similar to Schlögl's second model [97] of autocatalysis characterized by chemical reactions $2 X \longrightarrow 3 X, X \longrightarrow \emptyset$ where $X$ represents the reactant. Grassberger [98] studied a version of Schlögl's second model in which each site has a maximum occupancy of two and doubly occupied sites give birth to a neighboring vacant site. He found that the model shows a continuous phase transition in 2D.

Studies to date on the QCP have been limited to regular lattices in low dimen- 
sions. In this paper, we extend the study to complex networks. There are two ways to view the QCP on networks:

- as a model that replaces the linear birth rate of the contact process that has been extensively studied on networks $[99,100]$, by a quadratic birth rate.

- as an alternative model for the spread of rumors, fads and technologies such as smart phones in a social network. In sociology the requirement of more than a single 1 for the "birth" event is called complex contagion [101]. Also related are the threshold contact process [102] and models for the study of "cascades" [51]. The key difference here is that the QCP involves a death event that represents the loss of interest in the fad or technology and the rate for birth events is a function of the actual number and not the fraction of occupied neighbors.

The questions we are interested in are: How does network topology affect the phase transitions? What model and network features lead to discontinuous versus continuous phase transitions?

The rest of this chapter is organized as follows. We define the specific QCP that we study in Section 3.2 and we do mean field calculations in Section 3.3. In Section 3.4 we present a few rigorous results about the QCP. Simulation results are presented in Section 3.5, followed by some concluding remarks in Section 3.6.

\subsection{Model definition}

The birth event in the linear contact process can be formulated as each $1-0$ edge converts to a $1-1$ edge at a constant rate $\lambda$. Such a definition can be easily extended to the quadratic case by defining the birth event in terms of connected vertex triples. Two such definitions are possible: $1-0-1 \longrightarrow 1-1-1$, and $1-1-0 \longrightarrow 1-1-1$. We

call the former version the vertex centered QCP (VQCP) because the central 0 vertex 
is getting filled by its two neighboring 1s, and the latter as the edge centered QCP (EQCP) as it can be viewed as a $1-1$ edge giving birth on to a neighboring vacant vertex. Note that the models can also be defined in terms of how a vacant vertex gets filled i.e., suppose that a 0 vertex has $k 1$ neighbors and $j 1-1$ neighbors ${ }^{1}$, then the 0 vertex will become 1 at rates $\left(\begin{array}{l}k \\ 2\end{array}\right) \lambda$ and $j \lambda$ in the VQCP and EQCP respectively. Death events $1 \longrightarrow 0$ occur at rate 1 as in the linear process.

If the death rate is changed to zero, the VQCP reduces to bootstrap percolation [103], where vertices that are occupied remain occupied forever, and vacant vertices that have at least two occupied neighbors become occupied. While bootstrap percolation is typically defined in discrete time, the final configuration of the network is independent of whether the dynamics happens in discrete or continuous (as in our model) time.

We will use random graphs as models for complex networks on which the QCP is taking place. We will denote by $d$ the degree of a randomly chosen vertex in the network and the degree distribution by $p_{k}=\mathbb{P}(d=k)$. We are interested in networks with size $n \rightarrow \infty$ and where the vertex degrees are uncorrelated. The specific random graphs that we will consider are

- Random regular graphs $\mathrm{RR}(\mu)$ in which each vertex has degree $\mu$. Since everyone has exactly $\mu$ friends, this graph is not a good model of a social network. However, the fact that it looks locally like a tree will facilitate proving results.

- Erdös-Rényi random graphs $\mathrm{ER}(\mu)$ where each pair of vertices is connected with probability $\mu / n$. In the $n \rightarrow \infty$ limit, the degree distribution of the limiting graph is Poisson with mean $\mu$. This is a prototypical model for the situation in which the degree distribution has a rapidly decaying tail.

- Power law random graphs $\operatorname{PL}(\alpha)$ with degree distribution $p_{k}=c k^{-\alpha}$. We

\footnotetext{
1 The number of $1-1$ neighbors of a vertex $x$ is $|\{(y, z):\{x, y\},\{y, z\} \in E, z \neq x, s(y)=s(z)=1\}|$
} 
are particularly interested in graphs where the exponent $\alpha$ lies between 2 and 3, which has been found to be the case for many real world networks [3]. We construct our graphs using the configuration model, so the degrees are uncorrelated.

We will occasionally refer to RR and ER as homogeneous networks as their degree distributions are peaked around the mean, in contrast to PL where the distribution has a heavy tail.

\subsection{Mean field calculations}

We can attempt an analytical study of the dynamics by writing the equations for the various moments of the network. Let $g$ be a small graph labeled with 1's and 0's. We define the $g$-moment, written as $\langle g\rangle$, of a $\{0,1\}$ valued process on a graph $G$ as the expected number of copies of $g$ that exist in the set of all subgraphs of $G$. For example if $g=1-0-1$, we look at all the connected vertex triples in the network and count the ones where the center vertex is in state 0 and the other two vertices are in state 1 . We will write $\rho\left(\lambda, \rho^{(0)} ; t\right)$ as the density $\langle 1\rangle / n$ at time $t$ with a birth rate of $\lambda$ and an initial configuration where each vertex is independently occupied with a probability $\rho^{(0)}$. The order parameter for our phase transitions is the steady state density

$$
\rho_{*}\left(\lambda, \rho^{(0)}\right)=\lim _{t \rightarrow \infty} \rho\left(\lambda, \rho^{(0)} ; t\right) .
$$

We define the critical birth rate $\lambda_{c}$ as the birthrate above which there exists a stable steady state density that is greater than zero, i.e.,

$$
\lambda_{c}=\inf \left\{\lambda: \rho_{*}(\lambda, 1)>0\right\} .
$$

In the definition above, we chose $\rho^{(0)}=1$ since it has the best chance of having a positive limit. We also define a critical initial density $\rho_{c}$ as the minimum initial 
density required to reach a positive steady state density when the birth rate is infinite, i.e.,

$$
\rho_{c}=\inf \left\{\rho^{(0)}: \lim _{\lambda \rightarrow \infty} \rho_{*}\left(\lambda, \rho^{(0)}\right)>0\right\}
$$

From their definitions, it is straight forward to write the dynamical equations of $\langle 1\rangle$ for the VQCP and the EQCP,

$$
\frac{\mathrm{d}}{\mathrm{d} t}\langle 1\rangle=-\langle 1\rangle+\lambda \begin{cases}\langle 1-0-1\rangle & \text { for the VQCP } \\ \langle 1-1-0\rangle & \text { for the EQCP }\end{cases}
$$

If we were to write the equations for the third order moments that appear on the RHS of (3.4), those equation would involve still higher order moments. Continuing this way, we end up with an infinite series of equations that are not closed. Therefore we resort to a mean field approximation by assuming the states of neighbors of a vertex to be independent at all times.

\subsubsection{Homogeneous networks}

In the following we do a naive calculation that ignores the correlation between degree and occupancy, which should be reasonable for homogeneous networks. With these assumptions, $\langle 1-0-1\rangle$ will be $n \rho^{2}(1-\rho) \mathbb{E}\left[\left(\begin{array}{l}d \\ 2\end{array}\right)\right]$. Plugging this value into (3.4) we get

$$
\dot{\rho}=-\rho+\lambda \rho^{2}(1-\rho) \mathbb{E}\left[\left(\begin{array}{l}
d \\
2
\end{array}\right)\right] .
$$

Setting the RHS of (3.5) to zero gives a cubic equation whose roots are the possible steady state densities $\rho_{*}$. Clearly, zero is a trivial root of (3.5). The other two roots are

$$
\rho_{ \pm}=\frac{1}{2}\left[1 \pm \sqrt{1-\frac{\lambda_{c}}{\lambda}}\right]
$$

These solutions are real only when $\lambda>\lambda_{c}=4 / \mathbb{E}\left[\left(\begin{array}{l}d \\ 2\end{array}\right)\right]$. In the language of nonlinear dynamics, (3.5) exhibits a saddle node bifurcation at $\lambda_{c}$. It is easy to see that zero 


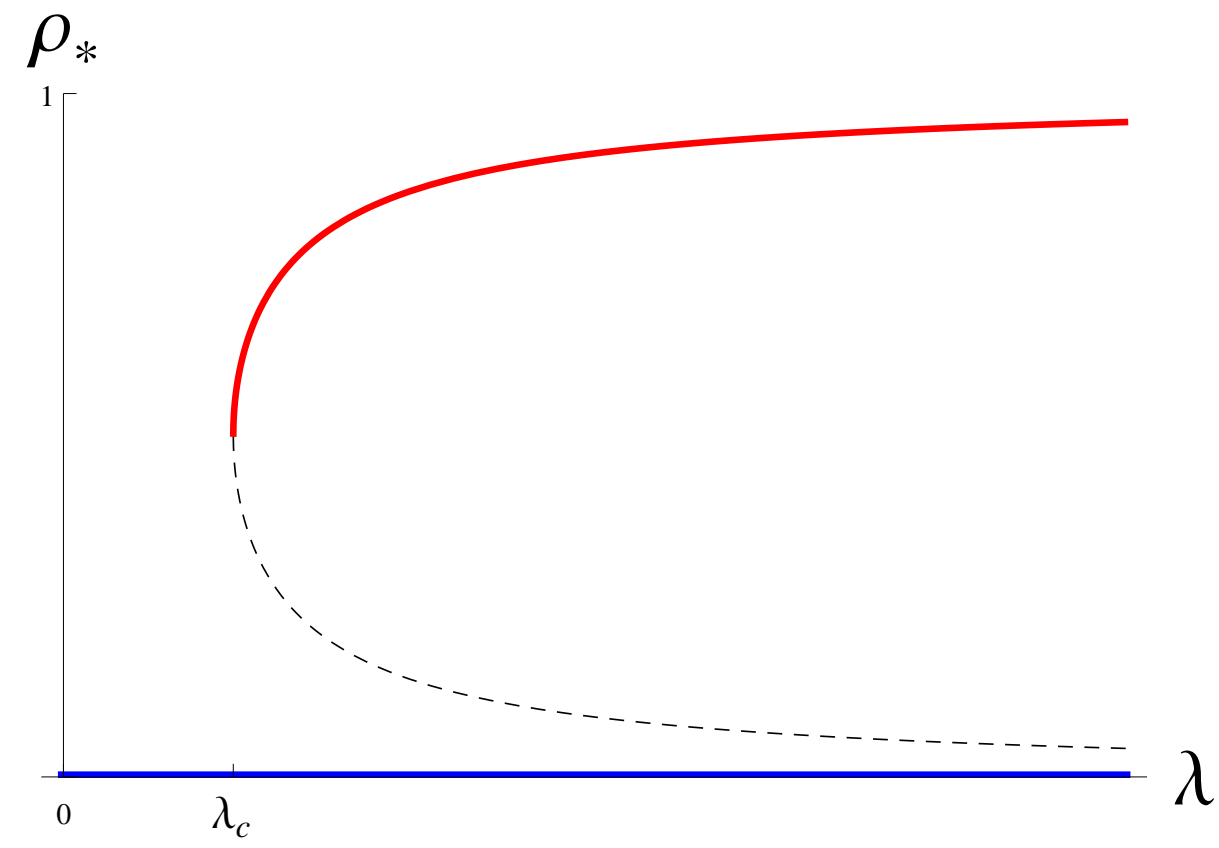

Figure 3.1: The solid red (top), dashed and solid blue (bottom) curves correspond to $\rho_{*}=\rho_{+}, \rho_{-}$and 0 respectively obtained from the mean field calculation for both $\mathrm{QCP}$ types on homogeneous networks.

and $\rho_{+}$are stable fixed points whereas $\rho_{-}$is an unstable fixed point. This can be seen in Fig. 3.1. The limiting critical initial density is

$$
\rho_{c}=\lim _{\lambda \rightarrow \infty} \rho_{-}=0
$$

For $\operatorname{ER}(\mu)$ we have $\mathbb{E}\left[\left(\begin{array}{l}d \\ 2\end{array}\right)\right]=\mu^{2} / 2$ which gives $\lambda_{c}=8 / \mu^{2}$. For $\operatorname{PL}(\alpha \leq 3)$ we have $\mathbb{E}\left[\left(\begin{array}{l}d \\ 2\end{array}\right)\right]=\infty$ so $\lambda_{c}=0$ while $\operatorname{PL}(\alpha>3)$ has finite $\mathbb{E}\left[\left(\begin{array}{l}d \\ 2\end{array}\right)\right]$ leading to a non-zero value for $\lambda_{c}$. The mean field calculation for the EQCP is essentially the same as done above and predicts the same qualitative features. Thus, for networks with finite $\mathbb{E}\left[\left(\begin{array}{l}d \\ 2\end{array}\right)\right]$, the simple mean field calculation predicts a discontinuous phase transition at $\lambda=\lambda_{c}$ and a region of bistability for $\lambda>\lambda_{c}$, for both QCP types. 


\subsubsection{Heavy tailed degree distributions}

The mean field calculation of Section 3.3.1 is simplistic since it ignores the fact that the occupancy probability depends on the degree. Pastor-Satorras and Vespignani [83] improved the mean field approach for the linear contact process by defining $\rho_{k}$, the fraction of vertices of degree $k$ that are occupied, and $\theta$, the probability that a given edge points to an occupied vertex. These variables can be related through the size biased degree distribution $q_{k}=k p_{k} / \mathbb{E}[d]$ which is the distribution of the degree of a vertex at the end of a randomly chosen edge.

$$
\theta=\sum_{k} q_{k} \rho_{k}
$$

Note that for homogeneous networks we assumed $\theta=\rho$. As before, the state of the neighbors of a vacant vertex are assumed to be independent. So the number of

occupied neighbors of a vertex of degree $k$ follow a distribution $\operatorname{Binomial}(k, \theta)$. This enables us to apply this approach to the VQCP. We write equations for $\rho_{k}$,

$$
\dot{\rho}_{k}=-\rho_{k}+\lambda\left(1-\rho_{k}\right)\left(\begin{array}{l}
k \\
2
\end{array}\right) \theta^{2}
$$

So in steady state

$$
\rho_{k *}=\frac{\lambda\left(\begin{array}{c}
k \\
2
\end{array}\right) \theta_{*}^{2}}{1+\lambda\left(\begin{array}{c}
k \\
2
\end{array}\right) \theta_{*}^{2}} .
$$

Combining (3.8) and (3.10) leads us to a self-consistent equation for $\theta_{*}$.

$$
\theta_{*}=\theta_{*} I\left(\lambda, \theta_{*}\right)
$$

where,

$$
I(\lambda, \theta)=\sum_{k=2}^{\infty} \frac{k p_{k}}{\mathbb{E}[d]}\left[\frac{\lambda\left(\begin{array}{l}
k \\
2
\end{array}\right) \theta}{1+\lambda\left(\begin{array}{l}
k \\
2
\end{array}\right) \theta^{2}}\right]
$$


Clearly, $\theta_{*}=0$ is a solution of (3.11). Finding a non-trivial solution involves solving

$$
I\left(\lambda, \theta_{*}\right)=1 \quad, \quad \theta_{*} \in(0,1) .
$$

Through the slighty lengthy calculation that follows, we show that for power law graphs $\operatorname{PL}(\alpha)$, the mean field calculation predicts:

- If $2<\alpha<3, \lambda_{c}=0$, the transition is continuous, and $\rho_{*}(\lambda) \sim \lambda^{\gamma(\alpha)}$.

- If $\alpha=3, \lambda_{c}>0$ and the transition is continuous.

- If $\alpha>3, \lambda_{c}>0$ and the transition is discontinuous.

Before attempting to solve (3.13), we note the following facts about it:

(i) $I(\lambda=0, \theta)=0 \forall \theta$, ruling out any solution to (3.13) when $\lambda=0$.

(ii)

$$
I(\lambda>0, \theta=1)=\sum_{k=2}^{\infty} \frac{k p_{k}}{\mu}\left[\frac{\lambda\left(\begin{array}{l}
k \\
2
\end{array}\right)}{1+\lambda\left(\begin{array}{l}
k \\
2
\end{array}\right)}\right]<\sum_{k=2}^{\infty} \frac{k p_{k}}{\mu}=1 .
$$

Since $I(\lambda, \theta)$ is continuous, this means that a solution to (3.13) will definitely exist if $\exists \lambda>0$ :

$$
I(\lambda, \theta \rightarrow 0) \geq 1
$$

(iii) The equality in (3.15) corresponds to the solution $\left(\lambda=\lambda_{c}, \theta \rightarrow 0\right)$.

(iv) If (3.15) is satisfied for $\lambda \rightarrow 0$, then $\left(\lambda=\lambda_{c}=0, \theta \rightarrow 0\right)$ is a solution.

(v) If (3.15) is not satisfied, then a $\theta$ that solves (3.13) is definitely positive. Also, $\exists \lambda=\lambda_{c}>0$ such that $\sup _{\theta \in(0,1)} I(\lambda, \theta)=1$. For $\lambda>\lambda_{c}$ there will be two roots, the larger of which is the relevant solution since we must have $\theta(\lambda)>\theta\left(\lambda_{c}\right)$.

(vi) If $\theta \rightarrow 0$ solves (3.13), then it corresponds to $\rho_{k} \rightarrow 0 \Rightarrow \rho \rightarrow 0$, and therefore the transition will be continuous. 
(vii) Conversely, if the solution $\theta$ is positive, then it corresponds to $\rho>0$, and consequently the transition will be discontinuous.

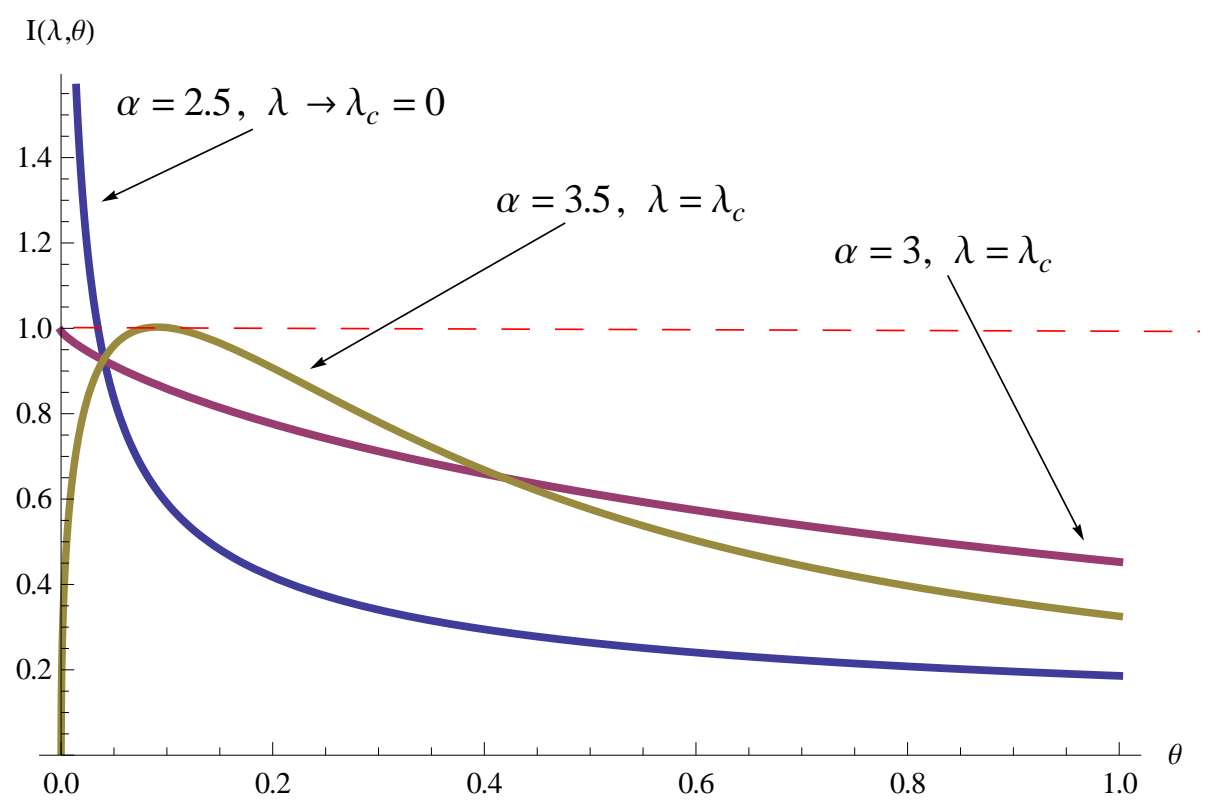

Figure 3.2: $I(\lambda, \theta)$ versus $\theta$ near $\lambda=\lambda_{c}$ for various power law graphs.

Substituing for $p_{k}$ in $(3.12)$,

$$
\lim _{\theta \rightarrow 0} I(\lambda>0, \theta)=\frac{c}{\mu} \lim _{\theta \rightarrow 0} \sum_{k=2}^{\infty} k^{1-\alpha}\left[\frac{\lambda\left(\begin{array}{l}
k \\
2
\end{array}\right) \theta}{1+\lambda\left(\begin{array}{l}
k \\
2
\end{array}\right) \theta^{2}}\right] .
$$

Converting the above sum to an integral by the substitution $x=k \theta$ and $\mathrm{d} x=\theta$

$$
\begin{aligned}
\frac{\mu}{c} \lim _{\theta \rightarrow 0} I(\lambda>0, \theta) & =\lim _{\theta \rightarrow 0} \sum_{k=2}^{\infty} k^{1-\alpha} \theta^{-2}\left[\frac{\lambda\left(\begin{array}{l}
k \\
2
\end{array}\right) \theta^{2}}{1+\lambda\left(\begin{array}{l}
k \\
2
\end{array}\right) \theta^{2}}\right] \theta \\
& =\lim _{\theta \rightarrow 0} \theta^{\alpha-3} \int_{0}^{\infty} x^{1-\alpha} \frac{\lambda x^{2} / 2}{1+\lambda x^{2} / 2} \mathrm{~d} x=\lim _{\theta \rightarrow 0} \theta^{\alpha-3} f(\lambda, \alpha),
\end{aligned}
$$

where,

$$
f(\lambda, \alpha)=\frac{\pi}{2^{\alpha / 2}} \csc [\pi(\alpha / 2-1)] \lambda^{\alpha / 2-1} \quad \text { if } \alpha \in(2,4),
$$


and $+\infty$ otherwise. Combining (3.17) and (3.18), we find that

$$
\lim _{\theta \rightarrow 0} I(\lambda>0, \theta)=+\infty \quad \text { if } \alpha \in(2,3)
$$

satisfying condition (3.15) $\forall \lambda>0$. Thus, combining facts (iv) and (vi), we conclude that $\lambda_{c}=0$ and the transition is continuous when $\alpha \in(2,3)$.

For small $\theta, I(\lambda, \theta) \sim \theta^{\alpha-3} f(\lambda, \alpha)$, so that solving $I(\lambda, \theta)=1$ gives

$$
\theta \sim[f(\lambda, \alpha)]^{1 /(3-\alpha)} .
$$

Now, $\rho=\sum_{k} p_{k} \rho_{k}$. For small $\theta$ which corresponds to small $\rho$, the sum can be converted into an integral:

$$
\begin{aligned}
\rho \sim \sum_{k=2}^{\infty} k^{-\alpha} \frac{\lambda\left(\begin{array}{l}
k \\
2
\end{array}\right) \theta^{2}}{1+\lambda\left(\begin{array}{l}
k \\
2
\end{array}\right) \theta^{2}} & \sim \theta^{\alpha-1} \int_{0}^{\infty} x^{-\alpha} \frac{\lambda x^{2} / 2}{1+\lambda x^{2} / 2} \mathrm{~d} x \\
& \sim \theta^{\alpha-1} f(\lambda, \alpha+1) \\
& \sim[f(\lambda, \alpha)]^{(\alpha-1) /(3-\alpha)} f(\lambda, \alpha+1) \sim\left(\lambda-\lambda_{\mathrm{c}}\right)^{\gamma(\alpha)},
\end{aligned}
$$

where the critical exponent

$$
\gamma(\alpha)=\frac{1}{3-\alpha}-\frac{1}{2}
$$

When $\alpha=3$, the limit $I(\lambda, \theta \rightarrow 0)=(\pi c / 2 \mu) \sqrt{\lambda / 2}$ is finite. Now using facts (iii) and (vi), we conclude that $\lambda_{c}=(2 \mu \sqrt{2} / \pi c)^{2}$ is positive, and the transition is continuous.

Moving on to the case of $\alpha>3$, we note that,

$$
I(\lambda, \theta) \lesssim \sum_{k} k^{1-\alpha} \frac{\lambda k^{2} \theta / 2}{1+\lambda k^{2} \theta^{2} / 2}=\sqrt{\lambda} b_{\alpha}(\theta \sqrt{\lambda / 2})
$$

where,

$$
b_{\alpha}(\epsilon)=\sum_{k} \frac{k^{3-\alpha} \epsilon}{1+k^{2} \epsilon^{2}}
$$


For $\alpha \in(3,4]$, we split sum in $b_{\alpha}(\epsilon)$ at $k=1 / \epsilon$, and write $b_{\alpha}(\epsilon)=b_{\alpha}^{\leq}(\epsilon)+b_{\alpha}^{>}(\epsilon)$.

$$
\begin{aligned}
& b_{\alpha}^{\leq}(\epsilon)=\sum_{k \leq 1 / \epsilon} \frac{k^{3-\alpha} \epsilon}{1+k^{2} \epsilon^{2}} \leq \epsilon \sum_{k \leq 1 / \epsilon} k^{3-\alpha} \\
& \leq \epsilon \int_{0}^{1 / \epsilon} x^{3-\alpha} \mathrm{d} x= \begin{cases}\frac{1}{4-\alpha} \epsilon^{\alpha-3} & \text { if } \alpha \in(3,4) \\
-\epsilon \log \epsilon & \text { if } \alpha=4\end{cases} \\
& \rightarrow 0 \text { as } \epsilon \rightarrow 0 \\
& b_{\alpha}^{>}(\epsilon)=\sum_{k>1 / \epsilon} \frac{k^{1-\alpha} \frac{k^{2} \epsilon^{2}}{1+k^{2} \epsilon^{2}}}{\epsilon} \leq \frac{1}{\epsilon} \sum_{k=1 / \epsilon+1} k^{1-\alpha} \\
& \leq \frac{1}{\epsilon} \int_{1 / \epsilon}^{\infty} x^{1-\alpha} \mathrm{d} k=\frac{1}{\alpha-2} \epsilon^{\alpha-3} \rightarrow 0 \text { as } \epsilon \rightarrow 0
\end{aligned}
$$

Thus, $b_{\alpha}(\epsilon) \rightarrow 0$ as $\epsilon \rightarrow 0$, which combined with (3.25) gives $I(\lambda, \theta \rightarrow 0)=0$. Now, using facts (v) and (vii), we conclude that $\lambda_{c}$ is positive and the transition is discontinuous when $\alpha \in(3,4]$.

For $\alpha>4$,

$$
b_{\alpha}(\epsilon) \leq \sum_{k} k^{3-\alpha} \epsilon \rightarrow 0 \text { as } \epsilon \rightarrow 0
$$

So, the nature of the transition is same as when $\alpha \in(3,4]$. This completes the calculation. The behavior of $I\left(\lambda \approx \lambda_{c}, \theta\right)$ for various values of $\alpha$ is shown in Fig. 3.2.

A second way to determine the nature of the phase transition is to adapt the argument of Gleeson and Cahalane [51], which can be applied if we use a discrete time version of the model in which a vertex with $k$ neighboring pairs will be occupied at the next step with probability $1-(1-\mathrm{p})^{k}$. The computation in their formulas (1)-(3) supposes that the vertices at a distance $\mathrm{n}$ from $x$ are independently occupied with probability $\rho_{0}$. The function $\mathbf{G}(\rho)$ defined in their (3) gives the occupancy probabilities at distance $k-1$ assuming that the probabilities at distance $k$ are $\rho$. 
Iterating $\mathrm{G} \mathrm{n}$ times and letting $\mathrm{n} \rightarrow \infty$ gives a prediction about the limiting density in the cascade. If one repeats the calculation for our system then 0 is an unstable fixed point when $\alpha<3$, while it is locally attracting for $\alpha>3$. This agrees with the mean-field prediction of $\lambda_{c}=0$ in the former case and a discontinuous transition with $\lambda_{c}>0$ in the second.

\subsection{Some rigorous results}

We have not been able to extend the mean field calculation to the EQCP on power law graphs, but by generalizing an argument of Chatterjee and Durrett [99] we can prove that $\lambda_{c}=0$ for $\alpha \in(2, \infty)$. The details are somewhat lengthy, so we only explain the main idea. Consider a tree in which the vertex 0 has $k$ neighbors and each of its neighbors has $l$ neighbors and $l$ is chosen so that $l \lambda \geq 10$. One can show that if $k$ is large then with high probability the infection will persist on this graph for time $\geq \exp (c(\lambda) k)$. In a power law graph one can find such trees with $k=n^{1 /(\alpha-1)}$. Using the prolonged persistence on these trees as a building block one can easily show that if we start with all vertices occupied the infection persists for time $\geq \exp \left(n^{1-\epsilon}\right)$ with a positive fraction of the vertices occupied. With more work (see $[104,105]$ ) one can prove persistence for time $\exp (c(\lambda) n)$.

For both types of QCP it is easy to show that it is impossible to have a discontinuous transition with $\lambda_{c}=0$. The proof for VQCP is as follows. Let $\left\langle 1_{k}\right\rangle$ be the expected number of occupied sites of degree $k$ and $\left\langle 10_{k} 1\right\rangle$ be the expected number of 1-0-1 triples when the 0 vertex has degree $k$. We can write an equation similar to

$$
\frac{\mathrm{d}}{\mathrm{d} t}\left\langle 1_{k}\right\rangle=-\left\langle 1_{k}\right\rangle+\lambda\left\langle 1-0_{k}-1\right\rangle
$$

which means at steady state 


$$
\begin{array}{r}
\left\langle 1_{k}\right\rangle_{*}=\lambda\left\langle 1-0_{k}-1\right\rangle_{*} \leq \lambda\left\langle 0_{k}\right\rangle_{*}\left(\begin{array}{l}
k \\
2
\end{array}\right) \\
\Rightarrow \rho_{k *} \leq \lambda\left(1-\rho_{k *}\right)\left(\begin{array}{l}
k \\
2
\end{array}\right) \Rightarrow \rho_{k *} \leq \frac{\lambda\left(\begin{array}{l}
k \\
2
\end{array}\right)}{1+\lambda\left(\begin{array}{l}
k \\
2
\end{array}\right)} .
\end{array}
$$

So, as $\lambda \rightarrow 0, \rho_{k *} \rightarrow 0$ and $\rho_{*}=\sum_{k} \rho_{k *} p_{k} \rightarrow 0$. Thus the transition will be continuous. The proof for EQCP is similar. In that case the subscript $k$ stands for the secondary degree $d^{(2)}$ which is defined as the number of neighbors of neighbors of a given vertex (not including itself), i.e., $d^{(2)}(x)=|\{z:\{x, y\},\{y, z\} \in E, z \neq x\}|$.

$$
\frac{\mathrm{d}}{\mathrm{d} t}\left\langle 1_{k}\right\rangle=-\left\langle 1_{k}\right\rangle+\lambda\left\langle 1-1-0_{k}\right\rangle
$$

So at steady state

$$
\left\langle 1_{k}\right\rangle_{*}=\lambda\left\langle 1-1-0_{k}\right\rangle_{*} \leq \lambda\left\langle 0_{k}\right\rangle_{*} k \Rightarrow \rho_{k *} \leq \frac{\lambda k}{1+\lambda k}
$$

Thus for both QCP types we find that if $\lambda_{c}=0$ then the phase transition is continuous.

For both QCP types on random $r$-regular graphs we can show that the critical birth rate is positive as follows. In the EQCP let there be $m$ occupied vertices. Each of these $m$ vertices can have at most $r$ neighbors that are vacant and can give birth on to them at rate $\leq(r-1) \lambda$ or die at rate 1 . So the total birth rate in the network is $\leq(r-1) \lambda r m$ against a death rate of $m$, and it follows that $\lambda_{c}>1 / r(r-1)$. Similarly, for the VQCP the total birth rate is $\leq \lambda\left(\begin{array}{l}r \\ 2\end{array}\right) r m$ and it follows that $\lambda_{c}>1 / r\left(\begin{array}{l}r \\ 2\end{array}\right)$. These arguments depend on the degree being bounded, so they do not work for Erdős-Rényi and power law graphs. 


\subsection{Simulation results}

We perform simulations of the QCP on $\mathrm{RR}(4), \mathrm{ER}(4)$ and $\mathrm{PL}(2.5)$. We generate the random regular and power law random graphs using the recipe called configuration model [12]. We draw samples $d_{x}$ from the degree distribution and attach that many "half-edges" to vertex $x$. We pair all the half edges in the network at random. We then delete all self loops and multiple edges. When $\alpha>2$ this does not significantly modify the degree distribution. If $\sum_{x} d_{x}$ turns out to be odd (an event with probabil-

ity $\left.\approx \frac{1}{2}\right)$, we ignore the last remaining unpaired half-edge. Furthermore, for PL(2.5) we start the degree distribution at 3 as, in the VQCP, the vertices of degree 1 and 2 are impossible or difficult to get occupied.

To deal with finite size effects, we observe how the plot of the steady state density $\rho_{*}(\lambda, 1)$ versus $\lambda$ starting with all vertices occupied changes when size $n$ of the network ranging from $10^{3}$ to $10^{5}$. Fig. 3.3 shows the results of both QCP types on $\mathrm{RR}(4)$ and ER(4). Here the curves seem to converge to a positive value implying a positive $\lambda_{c}$. The results for $\mathrm{PL}(2.5)$ are shown in Fig. 3.4. We observe that the transition happens close to zero and moves towards zero with increasing $n$ indicating that the critical birth rate is zero. As explained earlier, if $\lambda_{c}=0$ then the transition is continuous. This is consistent with the the mean field predictions for the VQCP and rigorous result for EQCP. In addition, in Fig. 3.4(a), the critical exponent for the $n=10^{5}$ curve can be measured to be approximately 1.45 which is close to the mean field value of 1.5 (obtained by setting $\alpha=2.5$ in (3.24)).

In order to further investigate the phase transitions in random regular and ErdösRényi graphs, we look at the steady state density attained by starting from two different initial densities for the same network size $n=10^{5}$. Fig. 3.5 again shows a similar pattern across both QCP and both network types. In Fig. 3.5(b) we see that for birth rates between 0.9 and 2.3, the VQCP survives when the starting 

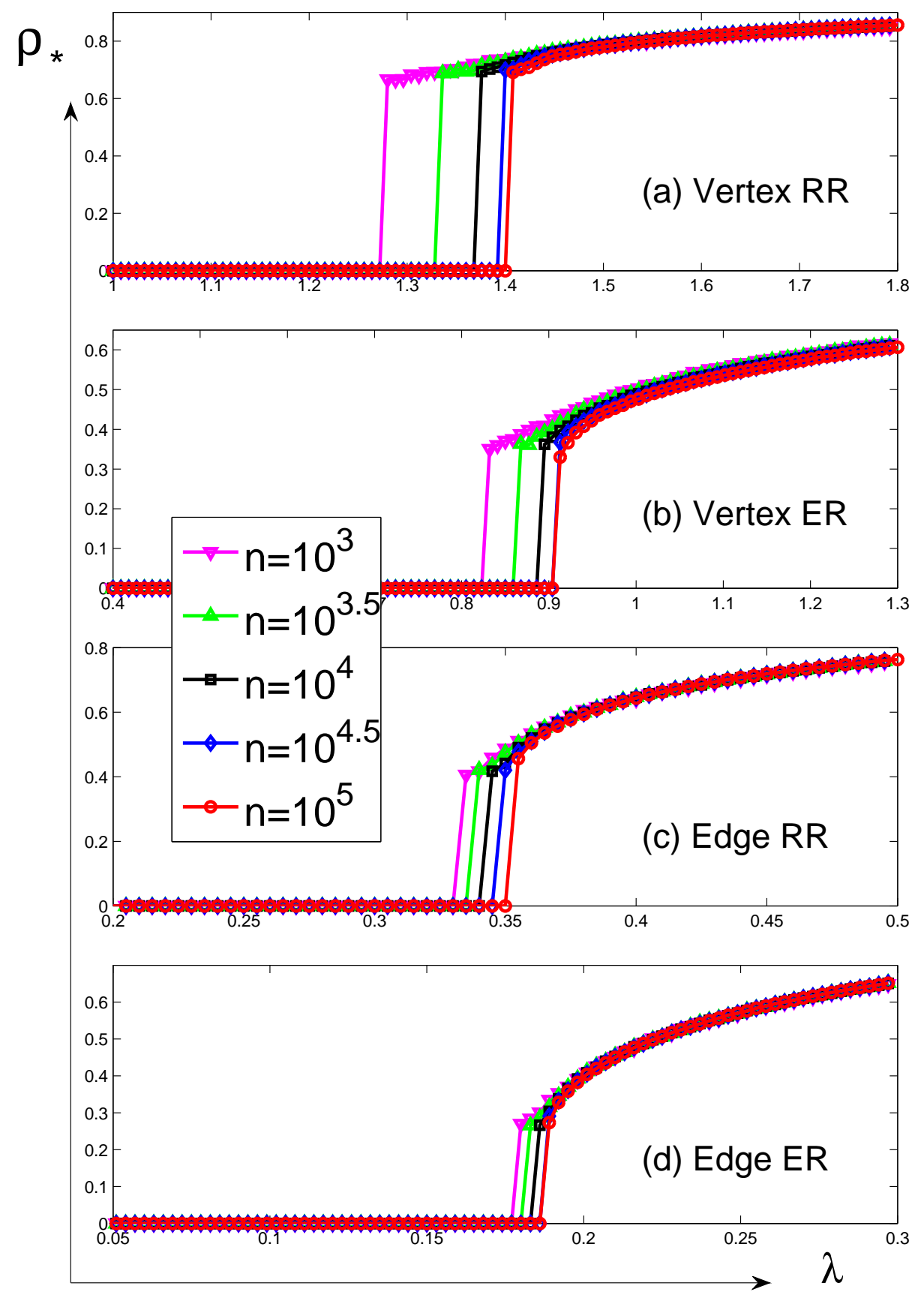

FiguRE 3.3: Steady state density reached, starting from all vertices occupied, for QCP on homogeneous networks of various sizes $n$.

configuration had all vertices occupied but dies out when starting with only onetenth of the vertices occupied. Thus we see bistability in the region $\lambda \in(0.9,2.3)$ implying a discontinuous transition and consequently that $\lambda_{c}$ is positive and close to 


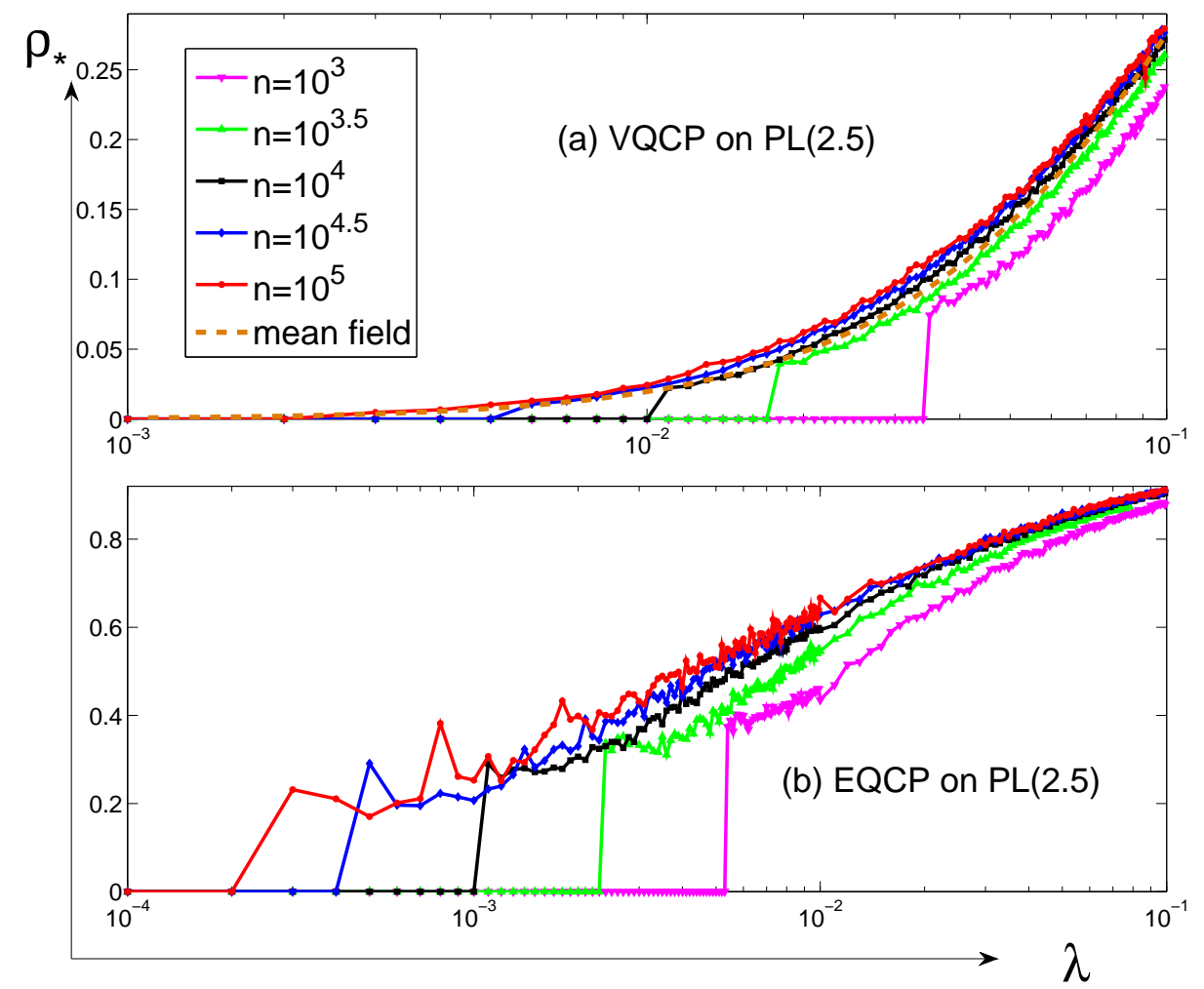

Figure 3.4: Steady state density reached, starting from all vertices occupied, for QCP on power law networks of various sizes $n$. Note that the $\lambda$ axis is in the log scale.

0.9. This is qualitatively in agreement with the mean field prediction seen in Fig. 3.1, although the critical birth rate of 0.9 shows a deviation from the mean field value of $8 / 4^{2}=0.5$

Fontes and Schonmann [106] have shown that for bootstrap percolation on the tree there is a critical density $p_{c}$ so that if the initial density is $<p_{c}$ then the final bootstrap percolation configuration has no giant component of occupied sites. In this situation having deaths at a positive rate in the VQCP will lead to an empty configuration. The last argument is for the tree, but results of Balogh and Pittel [107] show that similar conclusions hold on the random regular graph. While this argument is not completely rigorous, the reader should note that since all of the VQCP are dominated by bootstrap percolation, it follows that the limiting critical initial density 

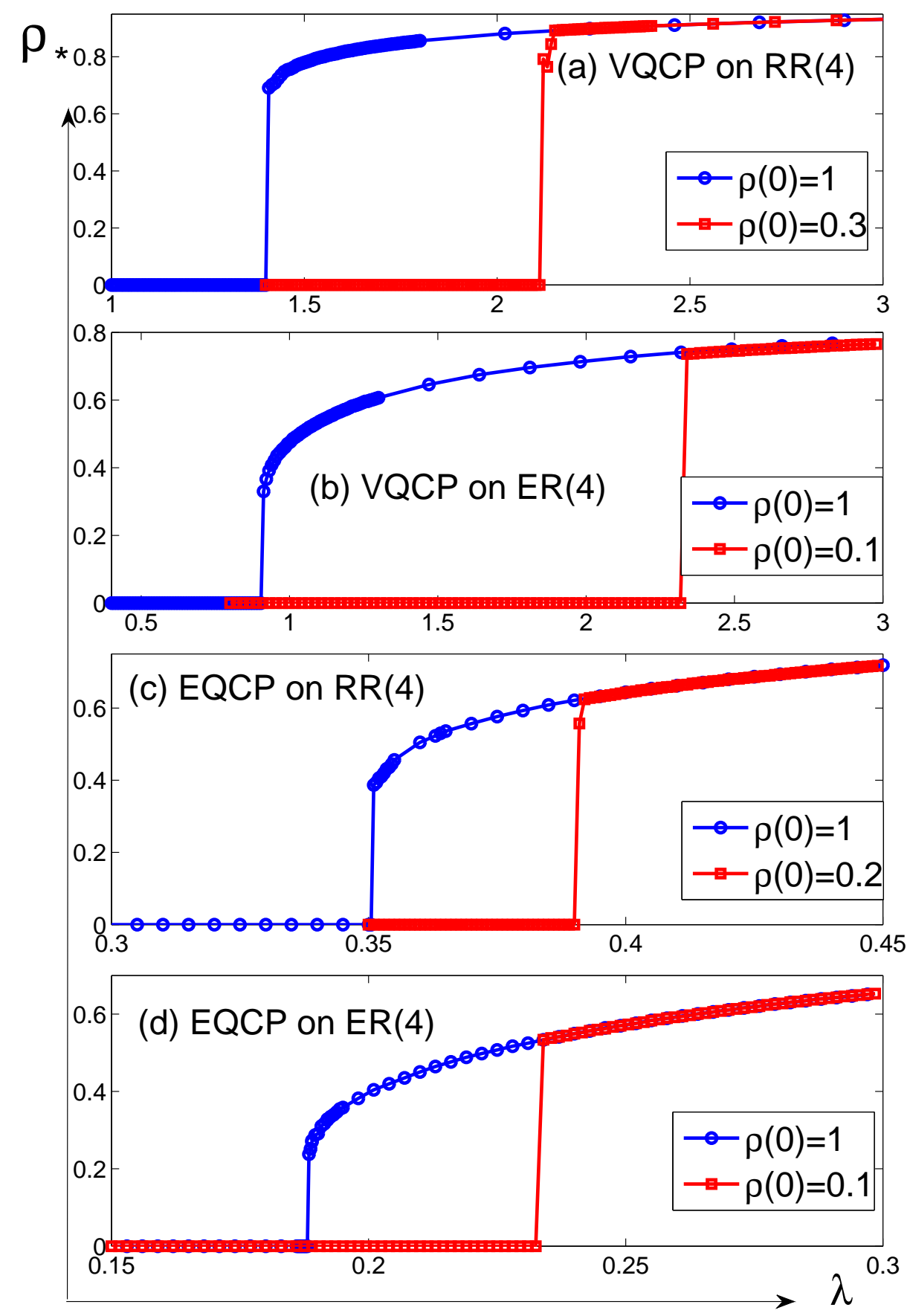

FiguRE 3.5: Steady state density reached, starting from two different initial densities $\rho^{(0)}$, for QCP on homogeneous networks of size $n=10^{5}$. Notice the similarity with the mean field prediction on Fig. 3.1.

defined in (3.3) has $\rho_{c}>0$ in contrast to the mean field prediction in (3.7). Fig. 3.6 shows the final density attained as a function of the initial density when the birth 


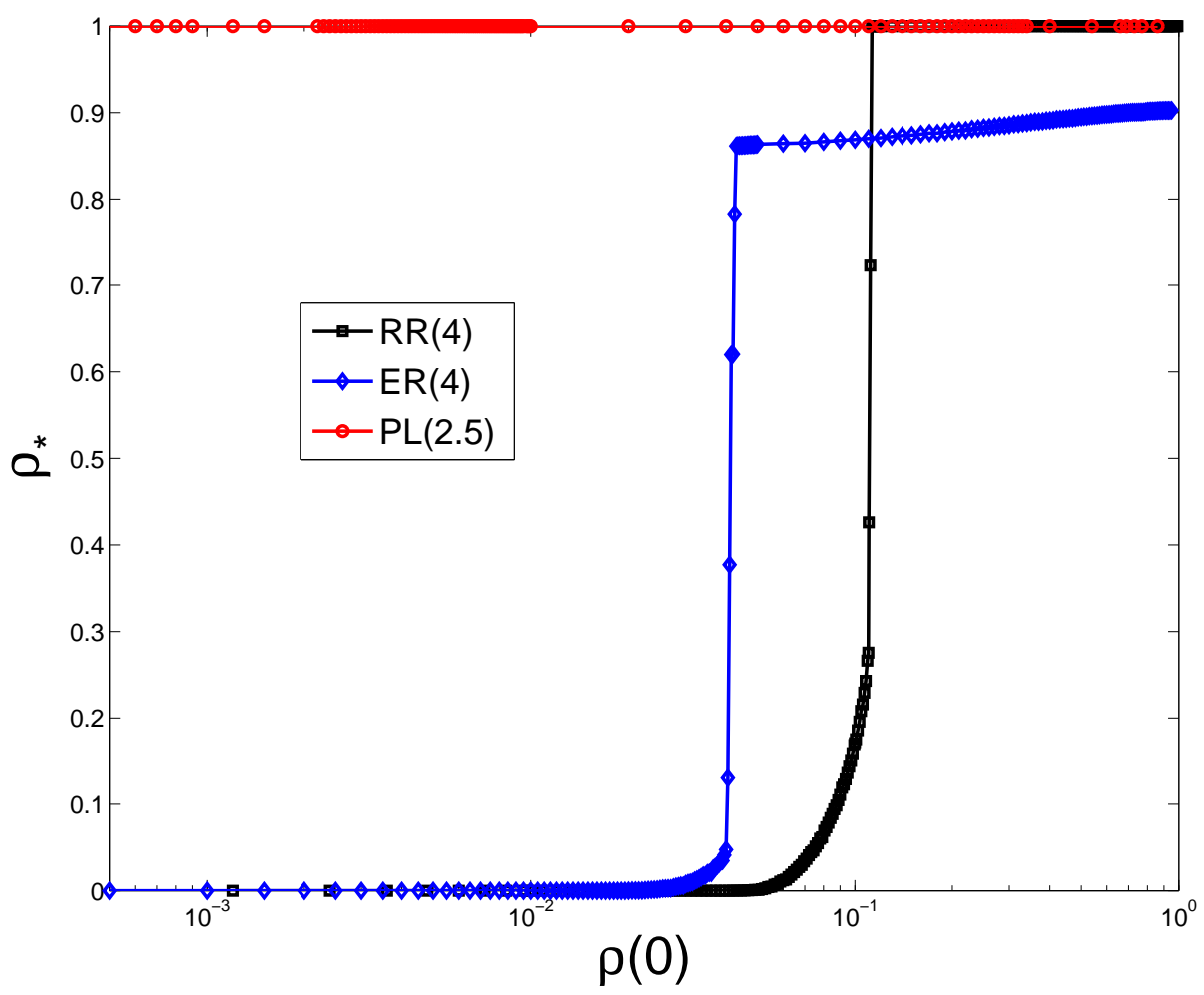

FiguRE 3.6: Steady state density when the birth rate is infinite in the VQCP on various networks of size $n=10^{5}$. Note that the $\rho^{(0)}$ axis is in the log scale.

rate is infinite (and death rate is positive). We see that $\rho_{c}$ in the VQCP is positive for the random regular and Erdős-Rényi graphs whereas it is zero for the power law graph. The corresponding results (not shown here) in the case of the EQCP indicate that $\rho_{c}=0$ for random regular, Erdős-Rényi and power law random graphs.

\subsection{Conclusion}

In this paper we have investigated the properties of two versions of the quadratic contact process on three types of random graphs. The mean field calculations we performed agree qualitatively with the simulation results. This may be due to the fact that complex networks have exponential volume growth and therefore are like infinite dimensional lattices where mean field is exact.

Table 3.1 summarizes what is known about the phase transitions of contact pro- 
Table 3.1: Nature of phase transitions of contact processes on various networks. Note that ' 0 ', '+' and ' $\infty$ ' stand for zero, positive and infinite values respectively of $\lambda_{c}$. The superscripts $\mathfrak{r}, \mathfrak{s}$, and $\mathfrak{m}$ indicate how the corresponding result was obtained - rigorously, by simulation, and by mean field calculation, respectively.

\begin{tabular}{|c|c|c|c|}
\hline & Linear CP & Vertex QCP & Edge QCP \\
\hline $1 \mathrm{D}$ & cont.,$+[79,108]$ & $\mathrm{NA}, \infty$ & cont. , + [109] \\
\hline $2 \mathrm{D}$ & cont.,$+[79,110]$ & discon.,$+[96]$ & cont. , + [98] \\
\hline $\mathrm{RR}$ & cont. [111] , + [112] & discon. $.^{\mathfrak{s} \mathfrak{m}},+^{\mathfrak{r} \mathfrak{s} \mathfrak{m}}$ & $\operatorname{discon} .^{\mathfrak{s m}},+^{\mathfrak{r} \mathfrak{s} \mathfrak{m}}$ \\
\hline ER & cont.,$+[100]$ & discon.,$+^{\mathfrak{s} \mathfrak{m}}$ & discon.,$+^{\mathfrak{s} \mathfrak{m}}$ \\
\hline$\overline{\mathrm{PL}}((2,3))$ & cont. , $0[99]$ & cont., $0^{\mathfrak{s m}}$ & cont., $0^{\mathfrak{r} \mathfrak{s}}$ \\
\hline $\mathrm{PL}(3)$ & cont. , 0 [99] & cont.,$+{ }^{\mathfrak{m}}$ & cont., $0^{\mathfrak{r}}$ \\
\hline $\operatorname{PL}((3, \infty))$ & cont. , 0 [99] & discon.,$+^{\mathfrak{m}}$ & cont., $0^{\mathfrak{r}}$ \\
\hline
\end{tabular}

cesses in 1 and 2 dimensional lattices and on the random graphs RR, ER and PL. The positivity of the critical birth rate for $1 \mathrm{D}, 2 \mathrm{D}$ and $\mathrm{RR}$ follows trivially from the boundedness of their degrees. For VQCP on a 1D lattice, two consecutive 0's can never get filled and it follows that $\lambda_{c}=\infty$. The results for the linear process on $\mathrm{RR}$ are inferred from the rigorous results for trees and the fact that $\mathrm{RR}$ is locally tree like.

The results indicate that the EQCP is qualitatively not very different from the linear contact process on low dimensional lattices and power law graphs, in contrast to the VQCP, which differs from its low dimensional analogue. In view of the fact that they are very different in how they fill vacant vertices on a network, the similarity between VQCP and EQCP in their phase transitions on complex networks is a little perplexing.

The EQCP can easily propagate on a chain and "cross bridges" connecting communities, compared to the VQCP which always requires two occupied neighbors. In the EQCP vertices with a large number of neighbors of large degree are the key to its survival. However, in the VQCP it is impossible for the central vertices to repopulate the leaves, so these structures are not long lasting. In contrast, the Gleeson-Cahalane calculation suggests that survival is due to the fact that as waves of particles move 
through the system the densities increase. 


\section{An Evolving Spatial Network model}

\subsection{Introduction}

The availability of real world network data spurred enormous interest in the study of complex networks starting in the late 1990s [81]. Numerous models have been proposed for the formation of many observed technological, social, information and biological networks. Many of these network models were purely topological, i.e., the location of the vertices of the network were irrelevant. However, it is clear that most real world networks have a spatial element to them. Examples include transportation networks [113-117], distribution networks [118], some social networks [119] and the neural network in the brain [120-122]. See [123] for an extensive review. The effects of space on the topology can be significant. For example, in a social network, individuals are likely to have more friends closer to their spatial locations than farther away.

Many of the models of spatial network that have been proposed are essentially static. Well studied models of this nature include the random geometric graph, the Waxman model of the internet [124], and the Watts-Strogatz model [8] that generates small-world networks. Barnett, Paolo and Bullock [125] performed an extensive study 
of networks where the probability that vertex pairs are connected depend on their spatial separation. Frasco et al. [119] studied a model for the formation of social networks where the topology was decided first and vertices were then sequentially placed in a square depending on the topology and distance to already placed vertices.

However, most real networks are not static but rather evolve in an attempt to improve their efficiency. For example, the networks in the brain are constantly rewired for the purpose of cognition and other brain functions $[10,126]$.

\subsubsection{A general evolution scheme}

We consider the equilibrium of evolving undirected spatial networks $G_{t}=\left(V, E_{t}\right)$, where $V=V_{n D}$ is a set of $n$ points uniformly distributed in a $D$-dimensional space $\mathcal{V}_{n D}$ of volume $n$, so that the mean density of points is unity. Although any metric can in principle be used, we will stick to the familiar Euclidean metric for defining distances. With applications to transportation and distribution networks in mind, the boundaries of the space are not periodic. We are primarily interested in the "thermodynamic" limit $n \rightarrow \infty$ and dimension $D=2$; so $\mathcal{V}_{n 2}$ is, say, a square of side $\sqrt{n}$. The network evolves only through the rewiring of edges, so the number of edges $\left|E_{t}\right|$ at time $t$, and consequently, the mean degree $\mu=2\left|E_{t}\right| / n$ are constant.

Consider a spatial network as defined above that is required to satisfy some topological constraint $\mathcal{T}$ (we only consider the constraint that the network is connected; however, other examples include: the network is planar, the degree of the vertices is bounded, etc.), and evolve with the aim of lowering its total length. Assume that the edges rewire independently of each other and according to the following Metropolis-Hastings dynamics [127]:

- Edges attempt to rewire at a rate proportional to some power $\delta \geq 0$ of their length. So if $\delta>0$, larger edges have a higher tendency to attempt to rewire. 
- If a proposed rewiring of an edge of length $\ell$ to an edge of length $\ell^{\prime}$ leads to a network that satisfies constraint $\mathcal{T}$, then it is accepted with a probability $\min \left(1, f(\ell) / f\left(\ell^{\prime}\right)\right)$, where $f(\cdot)$ is a non-decreasing function. In other words, a shorter edge is definitely accepted while the chance of a longer edge getting accepted is lower, the longer it is.

With the above evolution scheme, it is easy to find the distribution of the networks at equilibrium. Consider the set $\mathcal{G}$ of possible networks. Let $G, F \in \mathcal{G}$ and suppose that $F$ is formed by rewiring edge $\{x, y\}$ in $G$ to $\{x, z\}$. Without loss of generality assume $|x-y|>|x-z|$. The transition rates of going from $G$ to $F$ and from $F$ to $G$ respectively in one step are

$$
\begin{aligned}
& \Lambda(G \rightarrow F)=|x-y|^{\delta} \frac{1}{n-1-d(x)}, \quad \text { and } \\
& \Lambda(F \rightarrow G)=|x-z|^{\delta} \frac{1}{n-1-d(x)} \frac{f(|x-z|)}{f(|x-y|)}
\end{aligned}
$$

where $d(x)$ is the degree of vertex $x$. We seek an equilibrium distribution $\pi$ that satisfies detailed balance

$$
\boldsymbol{\pi}(G) \Lambda(G \rightarrow F)=\boldsymbol{\pi}(F) \Lambda(F \rightarrow G)
$$

This holds if $\boldsymbol{\pi}(G)$ is proportional to

$$
\prod_{\{x, y\} \in E} \frac{1}{|x-y|^{\delta} f(|x-y|)}=\exp \left[-\sum_{\{x, y\} \in E} \log \left(|x-y|^{\delta} f(|x-y|)\right)\right] \text {. }
$$

Note that all the transition rates and probabilities above are conditional on the vertex set $V_{n D}$. One may interpret the distribution (4.3) as follows: the cost of an edge is 
an increasing function of its length $\ell$, specifically, $\log \left[\ell^{\delta} f(\ell)\right]$; the cost of a network is the sum of the cost of its edges; the equilibrium networks have a distribution that is exponential in their cost.

The main motivation for our work is the model of segregation in a social network by Henry, Prałat and Zhang (HPZ) [37], which corresponds to the case $\delta>0, f(x)=$ constant in the general evolution scheme. They defined their model in discrete time with a parameter $p$ that controls the rate of convergence to equilibrium. Motivated by HPZ, Magura et al. [38] studied a continuous time model with $\delta=1$ and $f(x)=x^{\alpha-1}$.

\subsubsection{Our model}

In order to have short edges, we choose $f(x)=e^{\beta x}$, where $\beta$ is a non-negative parameter. For simplicity, we set $\delta=0$. In other words, edges make independent rewire attempts at a constant rate 1 , and longer edges are accepted with a probability that decays exponentially with the difference in the lengths. Thus, in our model, at each evolution step: an edge $\{x, y\} \in E$ is chosen at random and one of its vertices, say, $x$ is designated as its pivot; the vertex $x$ chooses another vertex $z$ outside its neighborhood; if the network created by rewiring the edge $\{x, y\}$ to $\{x, z\}$ satisfies constraint $\mathcal{T}$, then the move is accepted with probability $\min \left[1, e^{-\beta(|x-z|-|x-y|)}\right]$.

Substituting $\delta=0$ and $f(x)=e^{\beta x}$ in (4.3), we find our equilibrium network to be in the set $\mathcal{G}\left(V_{n D}, \mu, \mathcal{T}\right)$ of spatial networks with vertex set $V_{n D}$ and $n \mu / 2$ edges that satisfy constraint $\mathcal{T}$, and with a probability measure

$$
\boldsymbol{\pi}(G)=\frac{1}{Z_{\beta \mu}} e^{-\beta H(G)},
$$

where $H(G)=\sum_{\{x, y\} \in E(G)}|x-y|$ is the total length of the network, and $Z_{\beta \mu}=$ $\sum_{G \in \mathcal{G}} e^{-\beta H(G)}$ is a normalization constant. Thus, in going from the general evolution scheme to our model, we have made the definition of the cost of an edge more specific, 
i.e., the cost is proportional to its length, with the cost per unit length being $\beta$. With $n \rightarrow \infty$, the four parameters $D, \beta, \mu$ and $\mathcal{T}$ specify the equilibrium network of our Evolving Spatial Network model which we abbreviate as ESNM. The first two parameters $D$ and $\beta$ control the spatial effects, while the latter two $-\mu$ and $\mathcal{T}$ regulate the topology of the network.

\subsection{The unconstrained network}

In the simplest version of our model, the network is not required to satisfy any constraint. With this simplification, as we see below, the model is closely related to a percolation process and hence is amenable to some analytical calculations.

\subsubsection{A Fermion gas picture and connection with percolation}

In the unconstrained network, the distribution (4.4) of the equilibrium network leads us to an alternative view of the model. If we treat the $\left(\begin{array}{l}n \\ 2\end{array}\right)$ possible vertex pairs $\{x, y\}$ as the single particle energy levels $|x-y|$ in a Fermionic system, and the edges of the network to correspond to the occupied energy levels, then we have a non-interacting Fermionic system (constraints on the network would mean interacting Fermions). The parameter $\beta$ may then be viewed as the inverse temperature, $H(G)$ as the Hamiltonian of the system, and $Z_{\beta \mu}$ as the canonical partition function. However, this canonical ensemble description is analytically and computationally intractable. We will therefore use a grand canonical ensemble description which is equivalent to that of the canonical ensemble when the number particles is large. Given $V_{n D}$, the grand canonical partition function is

$$
\Xi(\beta, \kappa)=\sum_{G \in \mathcal{G}\left(V_{n D}\right)} \kappa^{|E(G)|} e^{-\beta H(G)},
$$

where $\kappa$ is the fugacity and $\mathcal{G}\left(V_{n D}\right)$ is the set of simple graphs with vertex set $V_{n D}$. 
Another way to simplify the equilibrium network, which is equivalent to the grand canonical description above, is to view it as the result of a percolation process on $V_{n D}$. For this, consider the set $\mathcal{G}\left(V_{n D}\right)$ of graphs as before, but now the edges assigned independently between all vertex pairs $\{x, y\}$ with probability $g(|x-y|)$. Barnett, Paolo and Bullock [125] studied such percolation networks for arbitrary functions $g(\cdot)$ and called them Spatially Embedded Random Networks. The distribution $\boldsymbol{\pi}^{\prime}$ of the percolation network is

$$
\begin{aligned}
\boldsymbol{\pi}^{\prime}(G) & =\prod_{\{x, y\} \in E} g(|x-y|) \prod_{\{x, y\} \in\left(\begin{array}{c}
V \\
2
\end{array}\right) \backslash E}(1-g(|x-y|)) \\
& =\left[\prod_{\{x, y\} \in\left(\begin{array}{c}
V \\
2
\end{array}\right)}(1-g(|x-y|))\right] \prod_{\{x, y\} \in E} \frac{g(|x-y|)}{1-g(|x-y|)},
\end{aligned}
$$

where $\left(\begin{array}{c}V \\ 2\end{array}\right)$ is the set of vertex pairs. This distribution can be made similar to $\boldsymbol{\pi}$ if we let

$$
\frac{g(\varepsilon)}{1-g(\varepsilon)}=\kappa e^{-\beta \varepsilon} \quad \text { which means } \quad g(\varepsilon)=\frac{1}{1+\kappa^{-1} e^{\beta \varepsilon}}
$$

As shown in [38], the properties of the percolation model are closely related to those of the random graph model if we choose $\kappa$ such that the expected mean degree in the percolation network is equal to the mean degree $\mu$ of our model. The only difference is that while the number of edges is fixed in the ESNM, it is random in the percolation version.

\subsubsection{Properties of the percolation network}

It is well known (see for e.g. [128]) that the grand partition function $\Xi(\beta, \kappa)$ of a collection of non-interacting particles at temperature $1 / \beta$ and fugacity $\kappa$, can be written as a product over single particle states. So, in our case, we have 


$$
\Xi(\beta, \kappa)=\prod_{\{x, y\} \in\left(\begin{array}{l}
V \\
2
\end{array}\right)}\left(1+\kappa e^{-\beta|x-y|}\right) .
$$

Since the partition functions above are conditional on the location of the vertices, we calculate the expected value

$$
\begin{aligned}
\mathbb{E} \log \Xi & =\left[\prod_{x \in V} \int_{\mathcal{V}_{n D}} \frac{\mathrm{d} x}{n}\right] \sum_{\{x, y\} \in\left(\begin{array}{l}
V \\
2
\end{array}\right)} \log \left(1+\kappa e^{-\beta|x-y|}\right) \\
& =\left(\begin{array}{l}
n \\
2
\end{array}\right) \int_{\mathcal{V}_{n D}} \int_{\mathcal{V}_{n D}} \frac{\mathrm{d} x}{n} \frac{\mathrm{d} y}{n} \log \left(1+\kappa e^{-\beta|x-y|}\right) .
\end{aligned}
$$

In the limit of large $n$, the double integration in (4.9) is to be performed over $\mathbb{R}^{2 D}$ and all points are identical. We can choose a point at $x$. Then calculate $\int g(|x-y|) \mathrm{d} y$, by constructing shells centered at $x$ at all radii $\varepsilon$. This integral will be independent of $x$. The remaining integral $\int \mathrm{d} x / n$ is just equal to 1 , giving

$$
\begin{aligned}
\mathbb{E} \log \Xi & =\frac{n}{2} S_{D-1} \int_{0}^{\infty} \varepsilon^{D-1} \log \left(1+\kappa e^{-\beta \varepsilon}\right) \mathrm{d} \varepsilon \\
& =\frac{n}{2} \frac{S_{D-1} \Gamma(D)}{\beta^{D}}\left[-\operatorname{Li}_{D+1}(-\kappa)\right] .
\end{aligned}
$$

Now that we know $\mathbb{E} \log \Xi$, the following two quantities can be directly calculated. First, the expected number of edges in the grand canonical ensemble is

$$
\begin{aligned}
\mathbb{E}|E(G)|=\mathbb{E}\left[\mathbb{E}\left[|E(G)| \mid V_{n D}\right]\right]=\mathbb{E} \frac{\partial \log \Xi}{\partial \log \kappa} & =\frac{\partial \mathbb{E} \log \Xi}{\partial \log \kappa} \\
& =\frac{n}{2} \frac{S_{D-1}}{\beta^{D}} \Gamma(D)\left[-\operatorname{Li}_{D}(-\kappa)\right],
\end{aligned}
$$


where $S_{D-1}=D \pi^{\frac{D}{2}} / \Gamma\left(1+\frac{D}{2}\right)$ is the area of the unit $(D-1)$-sphere, and $\operatorname{Li}_{s}(z)=$ $\sum_{k=1}^{\infty} z^{k} / k^{s}$ is the Polylogarithm function. In order that the grand canonical ensemble description be equivalent to the ESNM, we need to set $\mathbb{E}|E(G)|$ equal to the number of edges $n \mu / 2$ in the ESNM. This means

$$
\mu=\frac{S_{D-1}}{\beta^{D}} \Gamma(D)\left[-\operatorname{Li}_{D}(-\kappa)\right]
$$

In the rest of the section we will treat $\kappa=\kappa_{\beta \mu}^{(D)}$ to be implicitly defined through (4.14), and $g(\cdot)=g_{\beta \mu}^{(D)}(\cdot)$.

Second, the expected value of the network Hamiltonian, i.e., the expected total length of the network is

$$
\mathbb{E} H(G)=-\frac{\partial \mathbb{E} \log \Xi}{\partial \beta}=\frac{n}{2} \frac{S_{D-1} \Gamma(D+1)}{\beta^{D+1}}\left[-\operatorname{Li}_{D+1}\left(-\kappa_{\beta \mu}^{(D)}\right)\right]
$$

The mean edge length $\xi=\mathbb{E}[H(G) /|E(G)|]$. When $n \rightarrow \infty$, both $H(G) / n$ and $|E(G)| / n$ will converge to their respective limits, so that

$$
\xi=\xi_{\beta \mu}^{(D)} \rightarrow \frac{\mathbb{E} H(G)}{\mathbb{E}|E(G)|}=\frac{S_{D-1} \Gamma(D+1)}{\mu \beta^{D+1}}\left[-\operatorname{Li}_{D+1}\left(-\kappa_{\beta \mu}^{(D)}\right)\right]
$$

To find the distribution of vertex degrees and edge lengths it is more convenient to use the percolation picture. First, let us determine the degree distribution: Let $X$ be a randomly chosen vertex and let $Y$ be one of the other vertices. The probability that $X$ is connected to $Y$ is

$$
\mathbb{P}(\{X, Y\} \in E)=\int \mathbb{P}(\{X, Y\} \in E \mid Y=y) \mathbb{P}(Y=y)=\frac{1}{n} \int g(|X-y|) \mathrm{d} y
$$

The probability that $X$ is connected to exactly $k$ of the other $n-1$ vertices is 


$$
\begin{aligned}
\mathbb{P}(d(X)=k) & =\operatorname{Binomial}(n-1, \mathbb{P}(\{X, Y\} \in E) ; k) \\
& =\text { Binomial }\left(n-1, \frac{1}{n} \int g(|X-y|) \mathrm{d} y ; k\right) \\
& \rightarrow \text { Poisson }\left(\int g(|X-y|) \mathrm{d} y ; k\right) \text { as } n \rightarrow \infty .
\end{aligned}
$$

So a vertex $x$ has its degree distributed as Poisson $[\mu(x)]$, where $\mu(x)=\int g(|x-y|) \mathrm{d} y$.

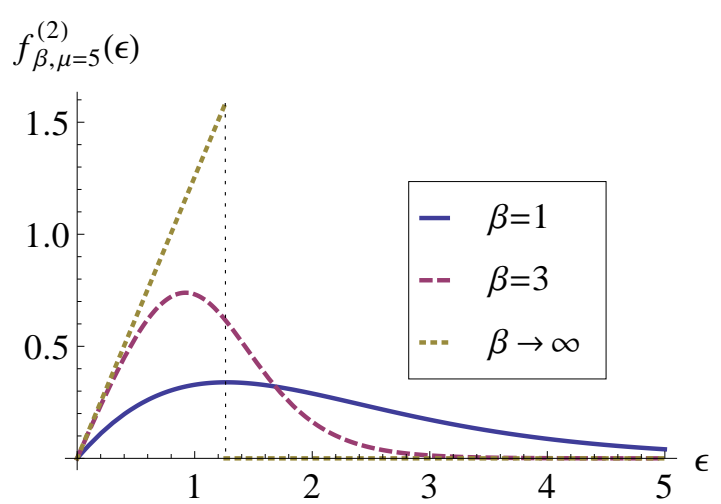

(a)

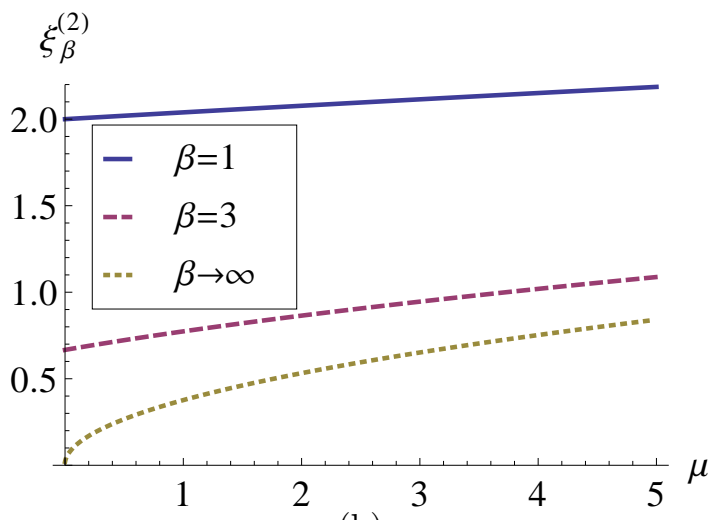

(b)

Figure 4.1: Results for $\beta=1$ (solid line), 3 (dashed line), and $\infty$ (dotted line) in dimension $D=2$; (a) gives the distribution of edge lengths when $\mu=5$, (b) gives the mean edge length as a function of $\mu$.

Next we consider the distribution of the lengths of the edges in the network. We want to find the probability

$$
\begin{aligned}
\mathbb{P}(|x-y|=\varepsilon \mid\{x, y\} \in E) & =\frac{\mathbb{P}(\{x, y\} \in E|| x-y \mid=\varepsilon) \mathbb{P}(|x-y|=\varepsilon)}{\mathbb{P}(\{x, y\} \in E)} \\
& =\frac{g_{\beta \mu}^{(D)}(\varepsilon) \mathbb{P}(|x-y|=\varepsilon)}{\int g_{\beta \mu}^{(D)}\left(\varepsilon^{\prime}\right) \mathbb{P}\left(|x-y|=\varepsilon^{\prime}\right)}
\end{aligned}
$$

As $n \rightarrow \infty, \mathbb{P}(|x-y|=\varepsilon) \rightarrow S_{D-1} \varepsilon^{D-1} \mathrm{~d} \varepsilon / n$. Substituting in (4.19), we get the probability density function of the distribution of edge lengths to be (see Fig. 4.1(a)) 


$$
f_{\beta \mu}^{(D)}(\varepsilon)=S_{D-1} \frac{g_{\beta \mu}^{(D)}(\varepsilon) \varepsilon^{D-1}}{\mu} .
$$

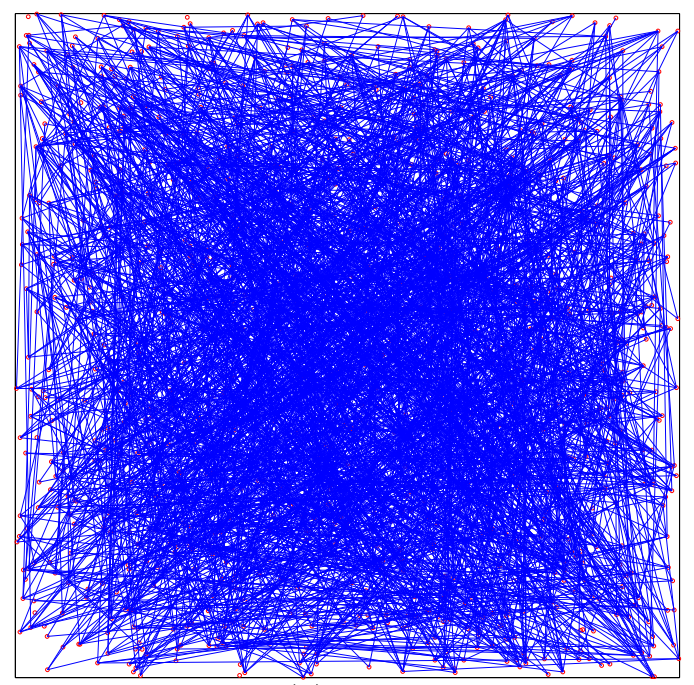

(a) $\beta=0$

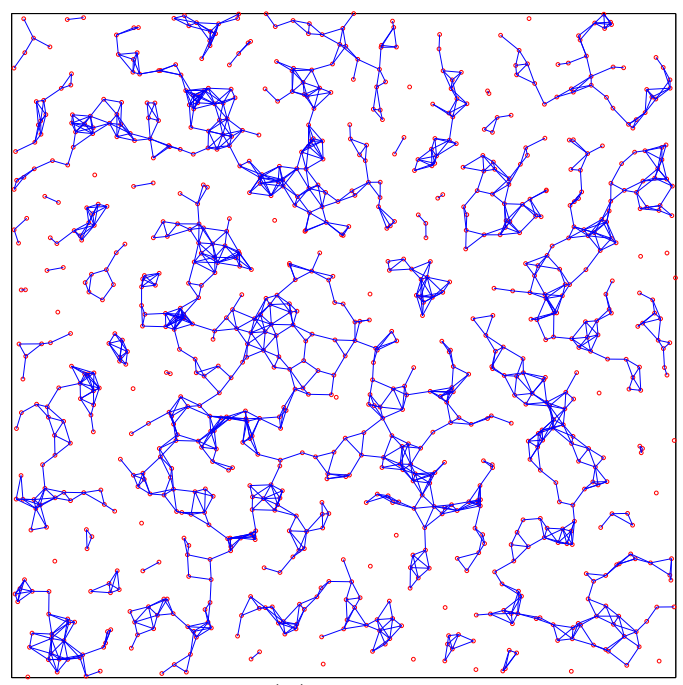

(b) $\beta \rightarrow \infty$

FiguRE 4.2: Pictures of the (a) Erdős-Rényi graph, and (b) the random geometeric graph, on a square with $n=1000$ vertices.

The two extreme values of $\beta$ deserve special consideration. With $\beta=0$, the spatial location of the vertices have no effect on the evolution of the network and so the equilibrium network is the Erdős-Rényi graph (a random graph drawn uniformly from the set of networks with a given number of vertices and edges) of which the degree distribution, the formation of the giant component, clustering coefficient, etc. are well known [1]. As $n \rightarrow \infty$, the mean edge length grows as the mean vertex pair distance which is $\mathcal{O}\left(n^{1 / D}\right)$. Note that this is consistent with the fact that $\lim _{\beta \rightarrow 0} \xi_{\beta \mu}^{(D)}=\infty$ for all $D, \mu>0$.

In the limit of large $\beta$, the rewiring algorithm becomes a greedy algorithm that always chooses the shorter edge. The equilibrium network will then be a random geometric graph $(\mathrm{RGG})[129,130]$ where vertices are connected to all their spatial 
neighbors up to a distance $\varepsilon_{0}$, equal to the $|E|$-th smallest distance between vertices. Alternatively, $\varepsilon_{0}$ is the Fermi energy ${ }^{1}$ of the system. This means

$$
\left(\begin{array}{l}
n \\
2
\end{array}\right) \mathbb{P}\left(|x-y|<\varepsilon_{0}\right)=|E|
$$

So as $n \rightarrow \infty$, we have

$$
\left(\begin{array}{l}
n \\
2
\end{array}\right) \int_{0}^{\varepsilon_{0}} \frac{1}{n} S_{D-1} \varepsilon^{D-1} \mathrm{~d} \varepsilon=n \frac{\mu}{2} \quad \text { which means } \quad \Omega_{D} \varepsilon_{0}^{D}=\mu
$$

where $\Omega_{D}=\pi^{\frac{D}{2}} / \Gamma(1+D / 2)$ is the volume of a unit $D$ - ball. The mean edge length is

$$
\xi_{\beta \rightarrow \infty, \mu}^{(D)}=\int_{0}^{\varepsilon_{0}} \varepsilon \frac{S_{D-1} \varepsilon^{D-1} \mathrm{~d} \varepsilon}{\Omega_{D} \varepsilon_{0}^{D}}=\frac{S_{D-1}}{(D+1) \Omega_{D}} \varepsilon_{0}=\frac{D}{(D+1) \Omega_{D}^{\frac{1}{D}}} \mu^{1 / D} .
$$

The clustering coefficient $C$ should be expected to be high for spatial networks, since two spatial neighbors of a vertex are also spatial neighbors of each other. Using three randomly chosen vertices $x, y$ and $z$, we find the clustering coefficient of the percolation network to be

$$
C=\mathbb{P}(\{x, z\} \in E \mid\{x, y\},\{y, z\} \in E)=\frac{\iint g(|x|) g(|y|) g(|x-y|) \mathrm{d} x \mathrm{~d} y}{\iint g(|x|) g(|y|) \mathrm{d} x \mathrm{~d} y}
$$

For general values of $\beta$, it is difficult to evaluate (4.24). However, in the $\beta \rightarrow \infty$ limit, $C$ can be calculated as done in [129]: Consider a vertex $z$ that is connected to vertices $x$ and $y$. This means that $x$ and $y$ lie within a $D$-ball of radius $\varepsilon_{0}$ centered at

\footnotetext{
${ }^{1}$ Recall that the Fermi energy of a Fermionic system is the highest occupied single particle energy level at zero temperature.
} 
$z$. Now, $x$ and $y$ will be connected to each other only if $y$ lies in the intersection of the $D$-balls of radii $\varepsilon_{0}$ centered at $x$ and $z$ respectively. In other words, the probability that $y$ is connected to $x$ is the ratio of the intersection volume of the $D$-balls to the volume of a $D$-ball. The volume of the cap that subtends a half angle $\theta$ of a unit $D$-ball is given by

$$
\Omega_{D}^{\text {cap }}(\theta)=\frac{\pi^{\frac{D-1}{2}}}{\Gamma\left(\frac{D+1}{2}\right)} \int_{0}^{\theta} \sin ^{D} t \mathrm{~d} t .
$$

If $|x-z|=\varepsilon<\varepsilon_{0}$, to find the intersection volume, we need to add the volumes of two such caps with $\theta=\arccos \left(\varepsilon / 2 \varepsilon_{0}\right)$. The probability that $|x-z|=\varepsilon$ is $S_{D-1} \varepsilon^{D-1} \mathrm{~d} \varepsilon / \Omega_{D} \varepsilon_{0}^{D}$. So averaging the intersection volume over all $\varepsilon$, we have,

$$
\begin{aligned}
C & =\frac{1}{\Omega_{D} \varepsilon_{0}^{D}} \int_{0}^{\varepsilon_{0}} 2 \Omega_{D}^{\operatorname{cap}}\left(\arccos \left(\frac{\varepsilon}{2 \varepsilon_{0}}\right)\right) \varepsilon_{0}^{D} \frac{S_{D-1} \varepsilon^{D-1} \mathrm{~d} \varepsilon}{\Omega_{D} \varepsilon_{0}^{D}} \\
& =\frac{2 D^{2}}{\sqrt{\pi}} \frac{\Gamma(D / 2)}{\Gamma((D+1) / 2)} \int_{0}^{1} \int_{0}^{\arccos (t / 2)} \sin ^{D} \tau \mathrm{d} \tau t^{D-1} \mathrm{~d} t .
\end{aligned}
$$

Notice that $C_{\beta \rightarrow \infty, \mu}^{(D)}$ is independent of the mean degree $\mu$ consistent with the simulation result in Fig. 4.4.

It does not seem possible to analytically compute the size of the giant component in the percolation network. Because of this, we simulate the percolation process. In all the results that follow, the dimension $D=2$, and, unless otherwise stated, the network size $n=10^{4}$.

For fixed $\beta$, when $\mu$ is varied, the equilibrium network undergoes a percolation transition (in the $n \rightarrow \infty$ limit), indicated by the fraction $\rho$ of vertices in the giant component. For $\beta=0$, we know that the critical mean degree $\mu_{*}^{(\beta=0)}=1$ for formation of the giant component (see Fig. 4.3(a)). Increasing $\beta$ makes the formation of the largest component difficult, as long connections are not favored. However, there 


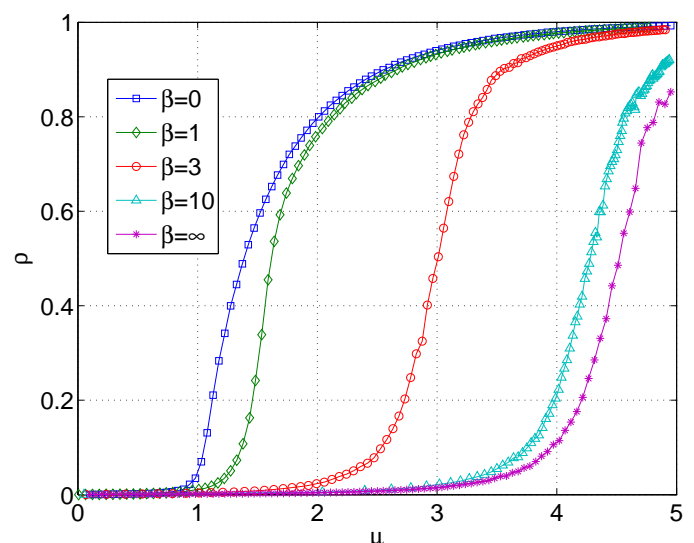

(a)

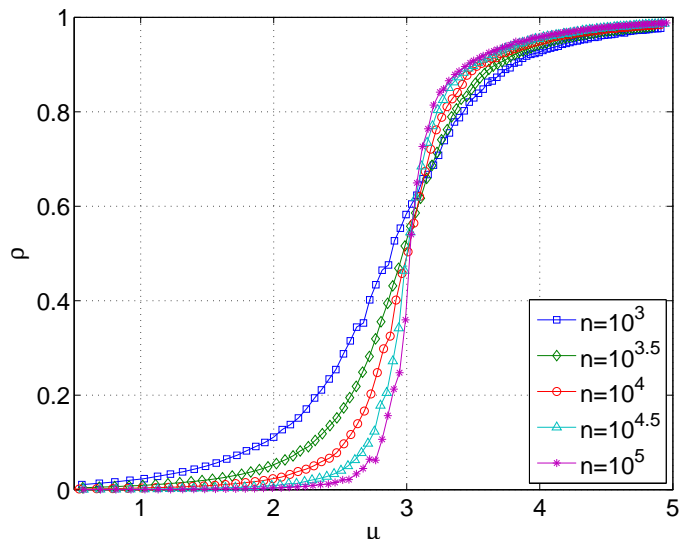

(b)

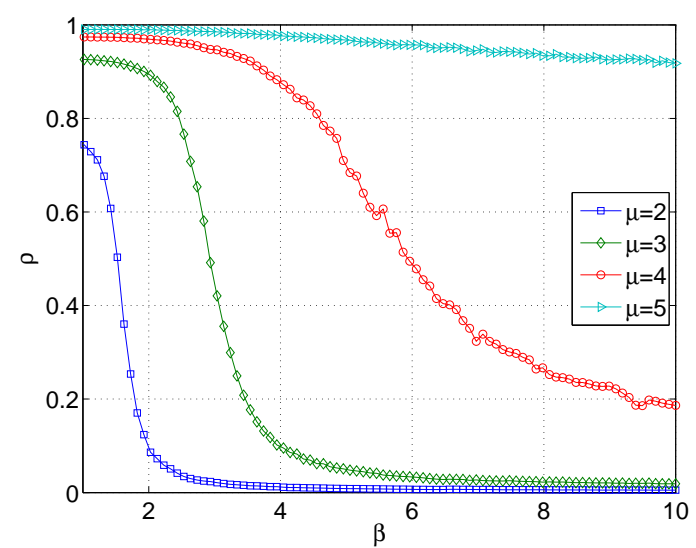

(c)

FiguRE 4.3: Fraction of vertices $\rho$ in the largest component (a) as a function of $\mu$ for various values of $\beta$ and (c) as a function of $\beta$ for various values of $\mu$. (b) shows the finite size scaling for $\beta=3$; notice that all the curves seem to cross at one point.

is an upper bound on $\mu_{*}$ achieved when $\beta \rightarrow \infty$ and the network is an RGG. Our simulation shows this bound to be $\mu_{*}^{(\beta \rightarrow \infty)} \approx 4.5$, in agreement with the simulation result reported in [129]. For $\beta=3$, the critical mean degree for percolation appears to be $\mu_{*}^{(\beta=3)} \approx 3.1$ from the crossing point of the curves corresponding to different network sizes in Fig. 4.3(b). Fig. 4.3(c) shows the size of the largest component as function of $\beta$ for fixed values of $\mu$. Consistent with Fig. 4.3(a), we see that for $\mu<\mu_{*}^{(\beta \rightarrow \infty)}$ there is a maximum $\beta=\beta_{*}$ for the existence of a giant component, while for $\mu>\mu_{*}^{(\beta \rightarrow \infty)}$ there is a giant component for all values of $\beta$. 


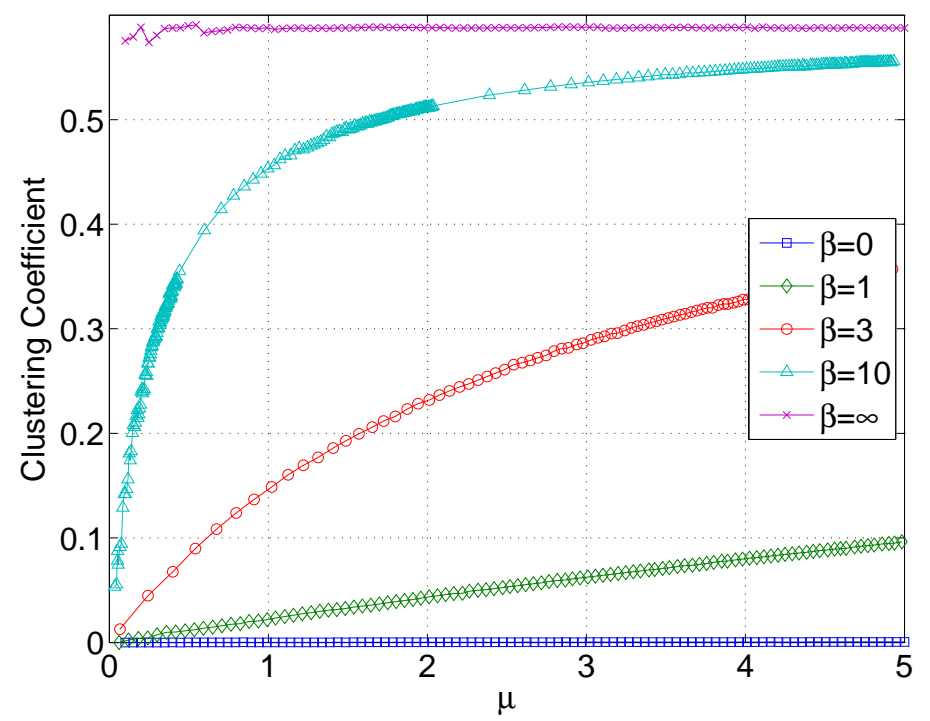

Figure 4.4: Clustering coefficient as a function of $\mu$ for various values $\beta$. (The non-uniform distribution of data points in $\mu$ correspond to uniform data points in $\kappa_{\beta \mu}^{(D)}$.)

For a given $\mu$, the clustering of vertices increases as $\beta$ increases, achieving the maximum value as $\beta \rightarrow \infty$ (see Fig. 4.4). Substituting $D=2$ in (4.26), we find that $C=1-3 \sqrt{3} / 4 \pi \approx 0.59$.

\subsubsection{A model for a social network}

The unconstrained model can be used as a model of a social network where the individuals have fixed opinions on $D$ number of issues. The parameter $\beta$ represents the tendency of individuals to befriend others of opinions similar to theirs, also known as homophily. The limitation in the number of active social contacts an average person can maintain is represented by the fixed value of the mean degree $\mu$. With the above interpretation of the parameters, the properties of the unconstrained ESNM are compatible with those of real social networks. First, clustering, which is a central feature of any social network is easily captured by the model (Fig. 4.4).

Second, the absence of a giant component would imply a fragmented social network (Fig. 4.3(a)). So the critical mean degree $\mu_{*}^{(\beta)}$ is the minimum number of friends 
that individuals need to make, so that the social network is mostly connected. The stronger the preference of individuals to connect to similar individuals (i.e., large $\beta$ ), the larger the number of friends they need to make (large $\mu$ ) to prevent disintegration. However, even with a very high homophily, if the number of friends is at least $\mu_{*}^{(\beta \rightarrow \infty)} \approx 4.5$, the social network is guaranteed to be mostly connected.

For $D=1$, the distribution (4.20) of edge lengths is monotonically decreasing, i.e., most edges are extremely small. This means than when there is only a single issue on which opinions matter, the individuals mostly connect to their closest spatial neighbors. However, for $D \geq 2$, the distribution has a maxima (Fig. 4.1(a)). So when individuals choose their friends based on their opinions on multiple issues, most of the friends are located farther away.

\subsection{The Connected ESNM}

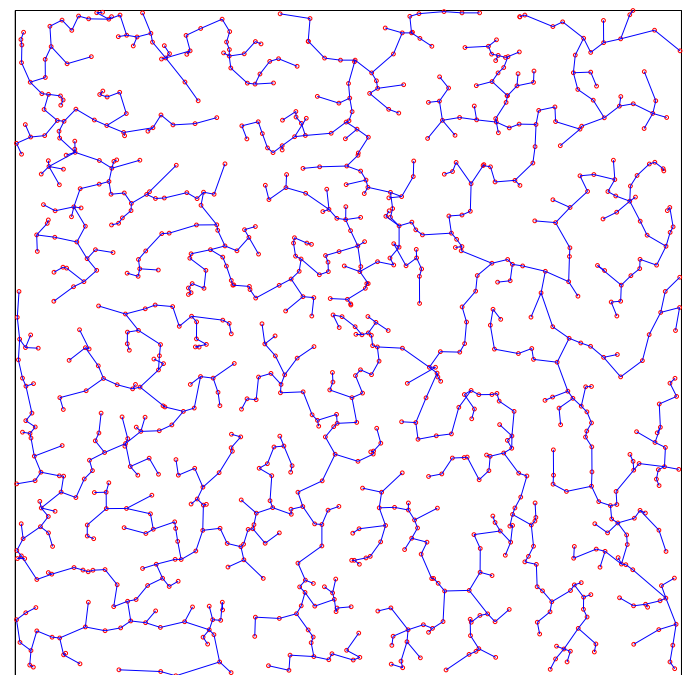

(a) $\mu=2$

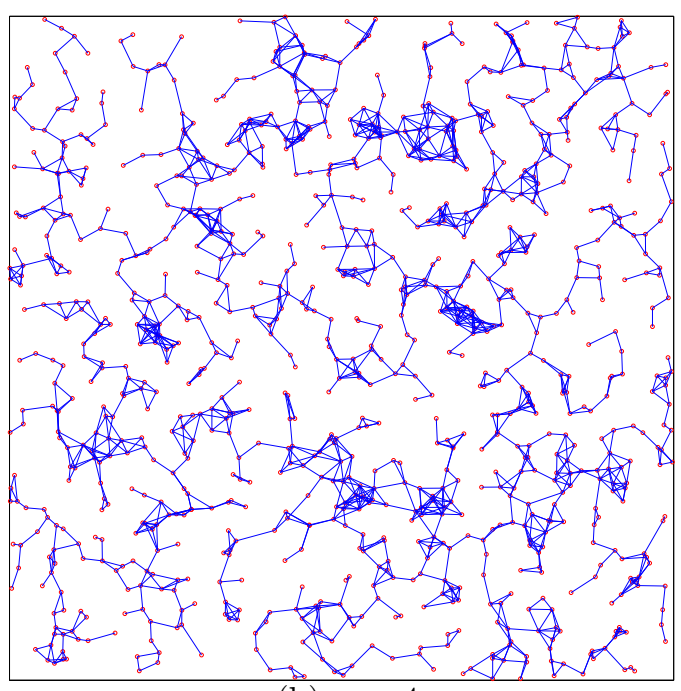

(b) $\mu=4$

FiguRE 4.5: A realization of the almost optimized network $(\beta=10)$ for two different values of the mean degree. The red circles and blue lines correspond to the vertices and edges respectively

We now consider our model with the constraint $\mathcal{T}$ that the network be con- 
nected. Such a requirement is natural for many real world networks, for e.g., airline networks [131]. Although, we know the equilibrium distribution (4.4) of the network, it is difficult to proceed further analytically as we can no longer define an equivalent percolation version of the model as we did for the unconstrained model. The connectedness constraint makes the edges of the equilibrium network highly correlated. Therefore we study the connected ESNM purely by simulation. For simplicity, we will focus on two cases: when the parameter $\beta$ is zero and when it takes a large value 10. We will refer to the $\beta=0$ equilibrium network as the Random Connected Network or $\operatorname{RCN}(\mu)$, and for reasons that will be elucidated in Section 4.3.1, the $\beta=10$ network will be called the Almost Optimized Network or $\operatorname{AON}(\mu)$.

In our simulations, we choose $n=10^{3}$ and dimension $D=2$. The initial network is formed by randomly ordering the vertices and adding $n-1$ edges to form a chain. The remaining $n \mu / 2-(n-1)$ edges are randomly chosen from the remaining vertex pairs. We say that equilibrium has been reached when the mean edge length changes by less that $0.5 \%$ across time points separated by a large number (1000 times the number of edges) of network update attempts. Fig. 4.5 shows the network for two values of the mean degree.

\subsubsection{The $\beta \rightarrow \infty$ model as an optimization process}

In the $\beta \rightarrow \infty$ limit, the connected ESNM may be viewed as a stochastic algorithm (although not a very efficient one) to solve the following optimization problem: Given a collection of $n$ points uniformly distributed in $\mathcal{V}_{n D}$ and $\mu \geq 2-2 / n$ (i.e., the number

of edges is at least the minimum $n-1$ needed to connect $n$ vertices), find the connected spatial network $G_{*}$ with mean degree $\mu$ that has the lowest total length, i.e., find

$$
G_{*}(\mu)=\underset{G \in \mathcal{G}_{c}\left(V_{n D}, \mu\right)}{\arg \max } H(G),
$$


where $\mathcal{G}_{c}\left(V_{n D}, \mu\right)$ is the set of connected networks with vertex set $V_{n D}$ and $n \mu / 2$ edges

Now, consider the general problem of finding an "efficient" network over a given collection of points. Obviously, the application one is interested in determines the optimization metric [132]. A simple and very popular optimal network is the minimum spanning tree abbreviated as MST (see [133] for a history and [134] for a classic algorithm). Here the quantity that is minimized is the total length, or equivalently, the "wiring cost" of the network. $G_{*}(\mu)$ is very similar to the MST with the notable exception that it is not a tree for $\mu \geq 2$. Indeed, $G_{*}(\mu=2-2 / n)$ is the MST.

However, one could potentially be concerned about other aspects of the network in addition to its wiring cost, and a tree may no longer be a good option. For example, Aldous $[135,136]$ sought networks which in addition to minimizing the wiring cost also has short routes, i.e., the route distance between any pair of vertices is close to their spatial distance. He quantified this property by defining the route factor $R(x, y)$ between two vertices $x$ and $y$ as

$$
R(x, y)=\frac{r(x, y)}{|x-y|}-1
$$

The route factor defined for a single vertex pair can then be averaged over all vertex pairs to arrive at a useful statistic for the network - the mean route factor $R$. Gastner and Newman [118] studied a growth model for spatial networks, where given a $V_{n 2}$ (i.e., vertices distributed uniformly in a square) with a designated "root" vertex, a connected cluster is grown by sequentially adding edges to vertices outside the cluster; the edges are chosen according to a greedy optimization criterion that minimizes a linear combination of the new edge length, and the route factor between the new and the root vertices.

One may also want the network to be robust to random failures of its edges. One way to test this kind of robustness of a connected network is by randomly removing 
edges [137] and noting the size of the largest component of the resulting network. Specifically, for a connected network of mean degree $\mu$ we look at the fraction $\rho_{\mu}\left(\mu^{\prime}\right)$ of vertices in the largest component when the edge removal leads to a network of mean degree $\mu^{\prime}$. A robust network should retain a large fraction of its vertices in its largest component when $\mu^{\prime}$ decreases from $\mu$; in other words the function $\rho_{\mu}\left(\mu^{\prime}\right)$ should be concave downwards for a sizeable region near $\mu^{\prime}=\mu$. We thus quantify the robustness of the network by the inflection point $\tilde{\mu}(\mu)$ of the $\rho_{\mu}(\cdot)$ curve. Note that lower $\tilde{\mu}(\mu)$ means more robust. The $\rho_{\mu}(\cdot)$ curve may always be convex indicating the lack of robustness of the network; therefore, for a collection of networks parametrized by their mean degrees $\mu$, we define the critical mean degree $\mu_{*}$ for robustness as the smallest $\mu$ for which there exists an inflection point.

Thus, similar to [116], we characterize the efficiency of a given network of mean degree $\mu$, by three statistics: the total edge length per vertex $\chi=H(G) / n=\xi \mu / 2$, the route factor $R$, and $\tilde{\mu}$. The smallness of all these network statistics is desirable for an efficient network. How does $G_{*}(\mu)$ fare in these measures of efficiency? In order to get an approximation to $G_{*}(\mu)$, we perform simulations using $\beta=10$ and term the equilibrium network as the almost optimized network or $\operatorname{AON}(\mu)$. Since the equilibrium of the $\beta=0$ connected model is uniformly drawn from the set $\mathcal{G}_{c}\left(V_{n D}, \mu\right)$ without regard for the edge length, it can be viewed as a null model for comparison with the AON, and we will refer to it as the Random Connected Network or $\operatorname{RCN}(\mu)$.

Although intuitive, it is not completely obvious that the route factor, and the total edge length will monotonically decrease and increase, respectively with the mean degree, as the simulations reveal. Fig. 4.6(a) shows that the route factor drops sharply as $\mu$ increases slightly from 2 . The two opposing statistics are plotted against each other in Fig. 4.6(b) to get convex "efficiency curves", which show that the AON is significantly more efficient than the RCN if we only take $\chi$ and $R$ into account.

However, in terms of robustness to random edge failures, the RCN with its abun- 


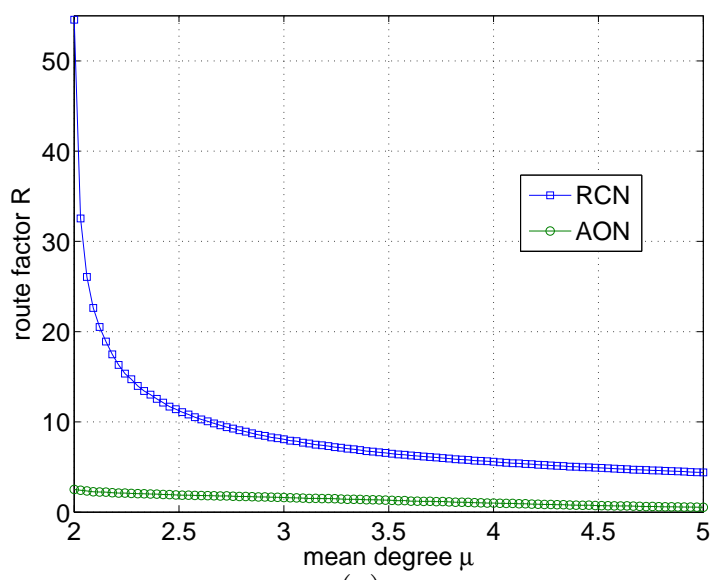

(a)

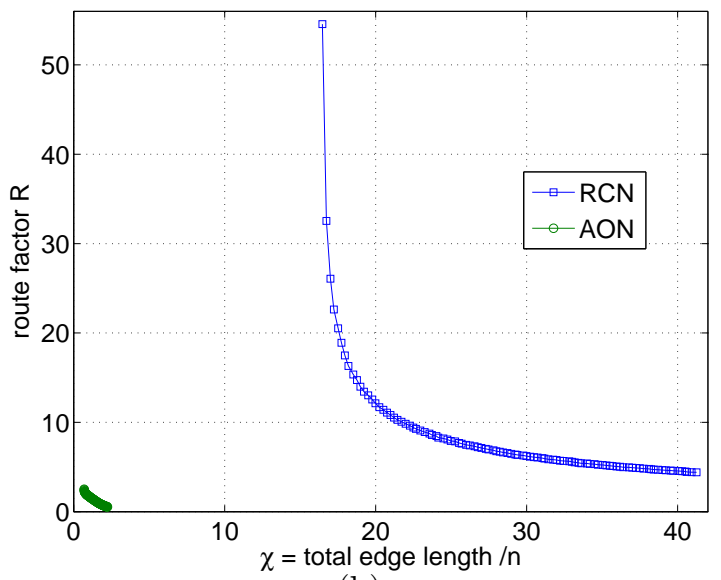

(b)

Figure 4.6: The route factor $R$ as a function of the (a) mean degree $\mu$, and (b) the total edge length per vertex $\chi$, for the RCN and AON.

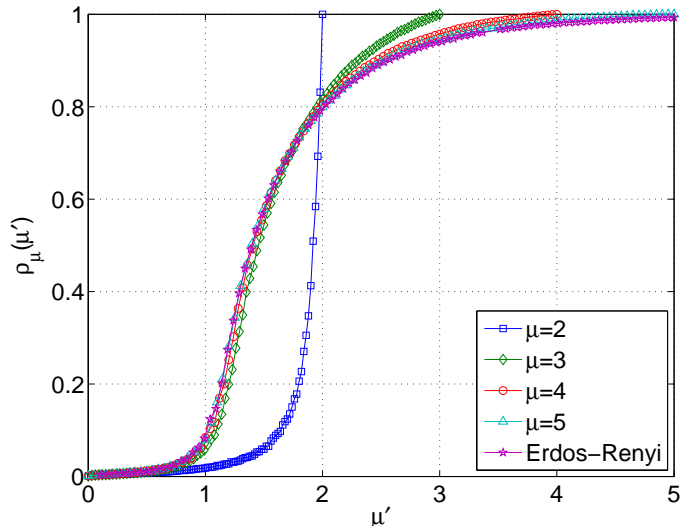

(a)

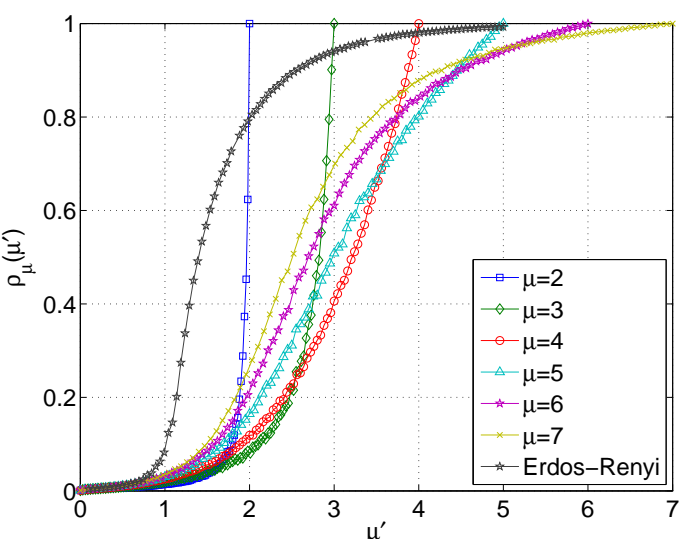

(b)

Figure 4.7: Robustness of the (a) RCN and (b) AON.

dance of long range connections performs better as shown in Fig. 4.7. For comparison, we also include the fraction of vertices in the largest component of an Erdős-Rényi random graph (note than an ER graph with edges removed at random is just another ER graph with a lower mean degree). While it is difficult to precisely locate the inflection point $\tilde{\mu}(\mu)$ in these curves, it is easy to see that it decreases with increase in the mean degree $\mu$, i.e., as one would expect, more edges make the network more robust. In Fig. 4.7(a), the $\mu=3,4,5$ curves are almost indistinguishable from 
the ER curve and show the percolation transition close to $\mu^{\prime}=1$, indicating that they are very similar to Erdős-Rényi networks. However, the behavior of the Almost Optimized Networks as seen in Fig. 4.7(b) is quite different. It can be inferred from Fig. 4.7 that the critical mean density $\mu_{*}=2$ for the $\mathrm{RCN}$, and $4<\mu_{*}<5$ for the AON.

\subsubsection{Other properties}

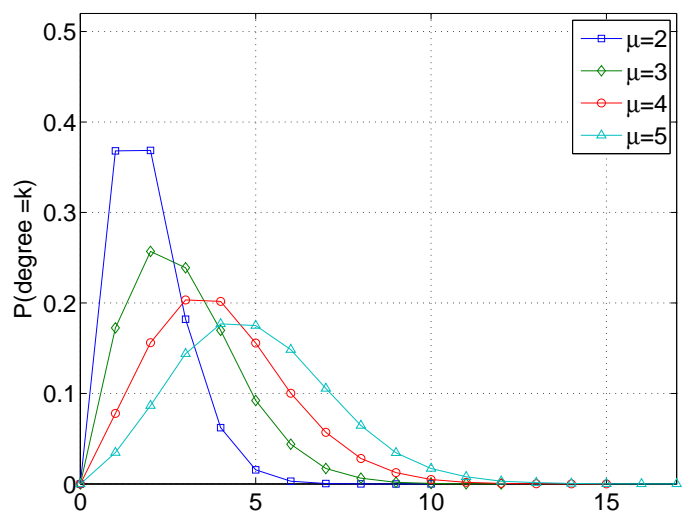

(a) $\stackrel{\mathrm{k}}{\mathrm{C}} \mathrm{N}$

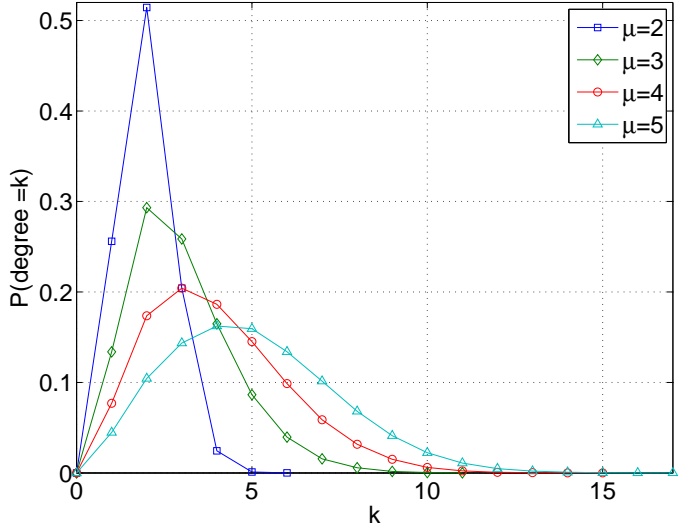

(b) $\mathrm{AON}$

FigURE 4.8: Degree distribution of the random connected and optimized networks.

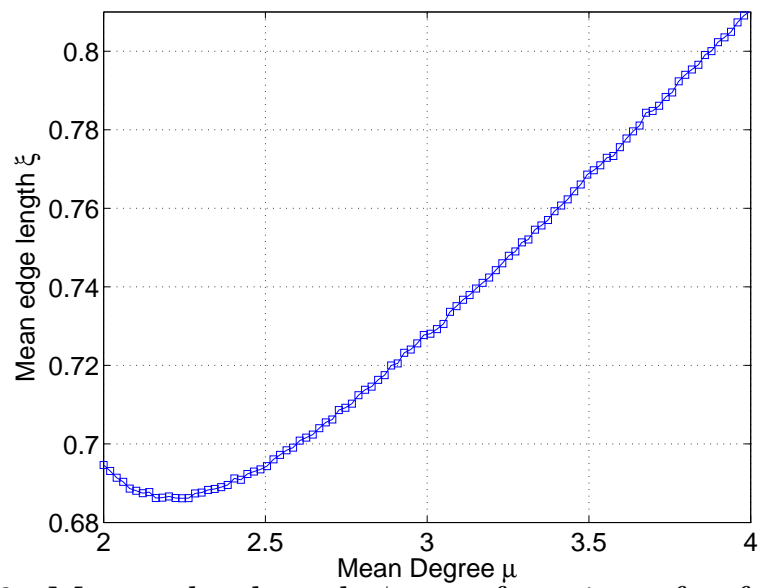

Figure 4.9: Mean edge length $\xi$ as a function of $\mu$ for the AON.

For the RCN, since the spatial locations are unimportant, it is natural that the clustering coefficient vanishes (as $n \rightarrow \infty$ ). The AON, on the contrary, has high 


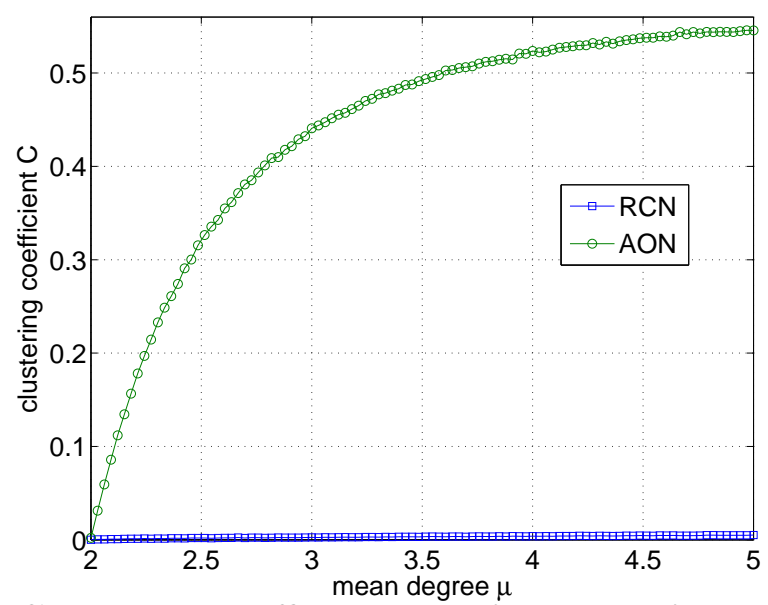

Figure 4.10: Clustering coefficient as a function of the mean degree $\mu$.

clustering as shown in Fig. 4.10.

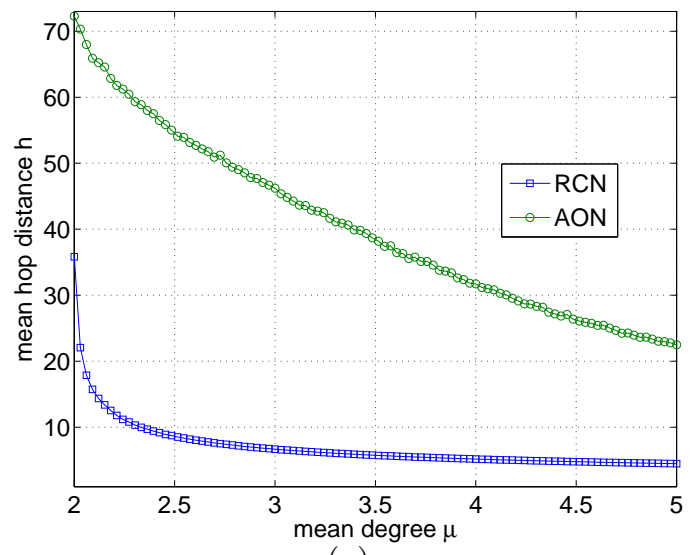

(a)

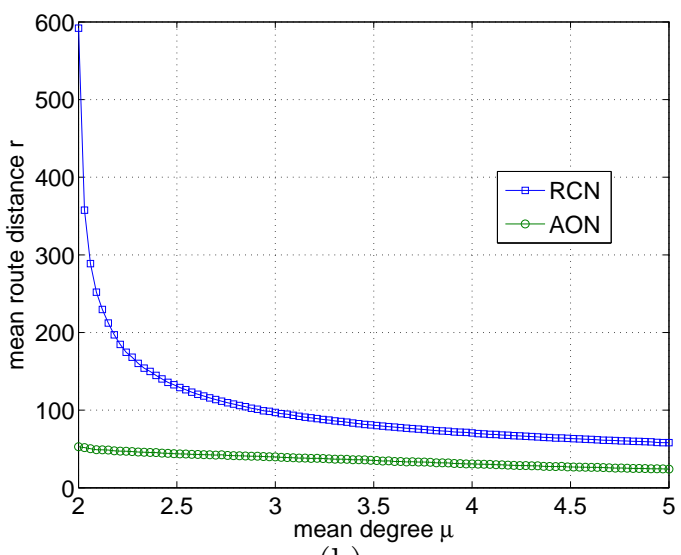

(b)

FiguRE 4.11: Hop distance $h$ and route distance $r$ as a function of the mean degree $\mu$ for the RCN and AON.

In contrast to the unconstrained network, the degree distribution of the connected network does not appear to be Poisson for any value of $\beta$, as seen in Fig. 4.8. The distribution however is still peaked around the mean with a thin tail. For $\mu=2$, the AON has a markedly higher peak at 2 than the RCN.

As in the unconstrained case, the mean edge length blows up in the $\mathrm{RCN}$ for $n \rightarrow \infty$. The mean edge length as a function of $\mu$ for the AON is shown in Fig. 4.9. It is interesting to note that that $\xi(\mu)$ is not monotone, but achieves a minima around 
$\mu=2.2$. As $\mu$ increases from 2 until about 2.5 , the increase in the total length of the network seems to be overcompensated by the increased flexibility in keeping the network connected, resulting in short edge lengths.

The mean route distance $r$ (Fig. 4.11(b)) is lower for the AON than for the RCN. However, in achieving a lower $r$, the AON gets a higher mean hop distance $h$ (Fig. 4.11(a)).

\subsubsection{Testing the model on real data}

In this section, we apply the connected ESN model on two sets of data, to gain some insight into the applicability of the model.

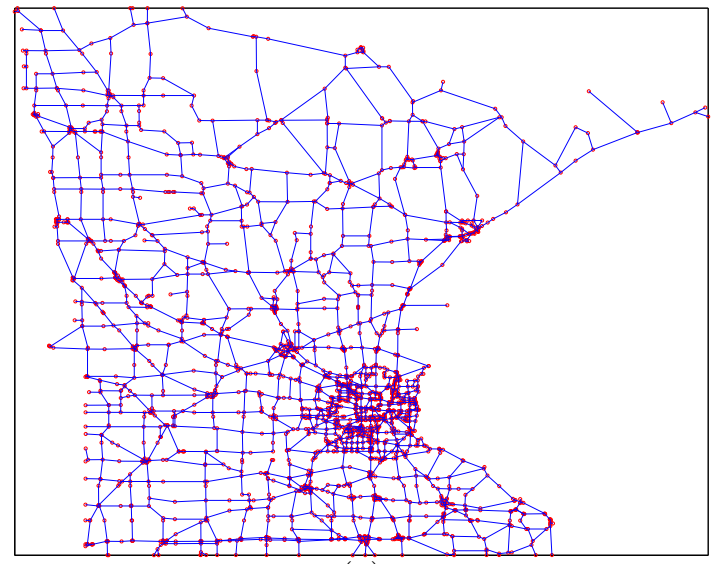

(a)

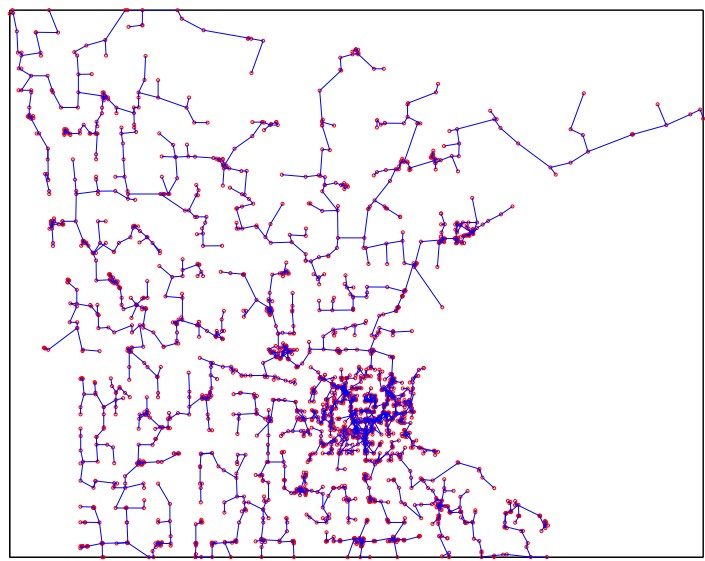

(b)

FiguRE 4.12: Pictures generated using the Minnesota road network data - (a) the actual network, and (b) the simulated network with $\beta=50$.

Table 4.1: Comparison of various statistics of the actual and simulated networks.

\begin{tabular}{|r|c|c|}
\hline & Data & Simulation \\
\hline mean edge length $\xi$ & 0.0682662 & 0.051706 \\
clustering $C$ & 0.0280275 & 0.113856 \\
hop distance $h$ & 80.0338 & 76.7535 \\
route distance $r$ & 6.10399 & 5.76164 \\
route factor $R$ & 1.78887 & 1.63773 \\
\hline
\end{tabular}




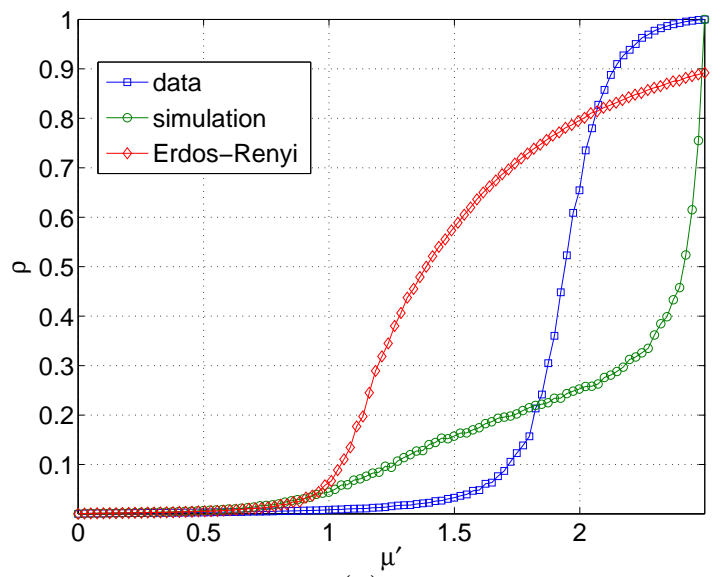

(a)

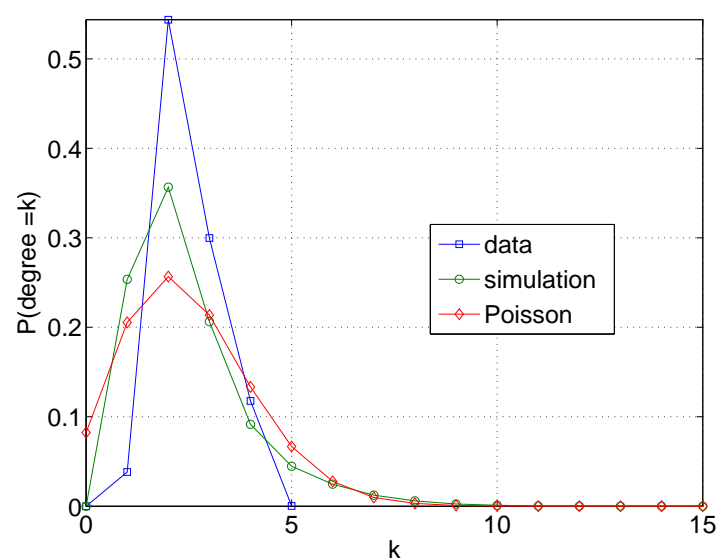

(b)

Figure 4.13: Comparison of (a) robustness and (b) degree distribution, of the actual, simulated, and Erdős-Rényi networks.

Our first data set is about the network of roads in the US state of Minnesota, obtained from [138] . There are $n=2635$ vertices in this network which correspond to the intersections of the roads. The mean degree is $\mu \approx 2.5$. To obtain the simulated network, we run the connected ESNM on the vertex set of the actual network, with the same mean degree and a large $\beta=50$.

In Fig. 4.12 we see that the actual and simulated networks look very different. While the actual network has a grid like structure almost throughout, the simulated network is tree like for the most part, expect for the small region (which corresponds to the capital city of Minneapolis) with a very high density of vertices. Table 4.1 compares the two network using the statistics we used earlier, and we find that the simulated network performs better than the actual network on all of them. Specifically, the hop and route distances, and the route factor, which are all measures of the ease of traversing the network, are marginally lower. Also for the simulated network, the construction cost of the roads measured by the mean edge length is slightly lower, while the clustering coefficient is significantly higher - a desirable feature.

So does this mean that the simulated network is the more "efficient" and "better" network? It does not seem likely that people living along the border with Canada 
would agree. They often have to go large distances to get to a nearby town, and even if they are driving to Minneapolis they have a much longer route. Second, how robust are the two networks to edge failures? Fig. 4.13(a) shows that the simulated network is extremely fragile compared to the actual network; a loss of less than $8 \%$ of the edges is enough to bring the size of the largest component down to a third of the network size. The actual network, on the contrary, is robust (by our earlier definition) with a $\tilde{\mu} \approx 1.9$. Fig. $4.13(\mathrm{~b})$ shows that in the actual network, a large fraction of the intersections are created by two roads, and no intersection is made of more than four roads - both of which are unsurprising. The simulated graph, while having a peak at 2, has unrealistic 9-road intersections in the capital region.

The confounding result above can be attributed mainly to the highly non-uniform distribution of vertices in the graph (recall that our model assumes a uniform distribution of the vertices). Our connected ESNM allocates a disproportionate amount of edges to regions of high vertex density. One may also argue about the quality of the statistics we used; specifically, in practical applications, extreme values of the hop and route distances and route factor are perhaps more relevant than their averages. Nevertheless, our robustness measure seems to be a reliable statistic for most cases.

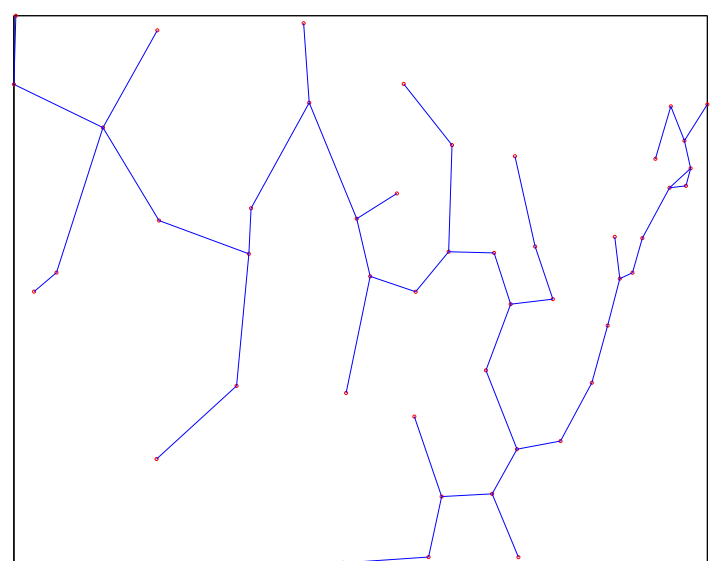

(a) $\mu=2$

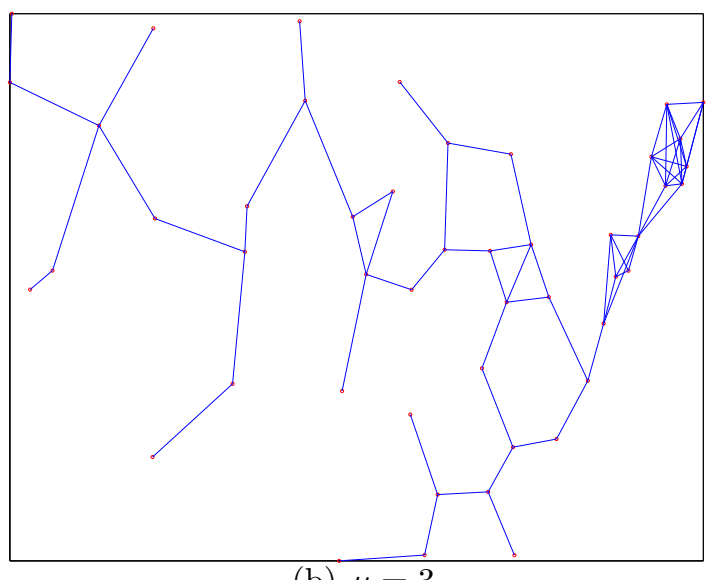

(b) $\mu=3$

Figure 4.14: Pictures of connected ESNM on US the state capitals" locations for two values of the mean degree. 
Table 4.2: Statistics obtained for the ESNM on US state capitals' locations with three values of the mean degree.

\begin{tabular}{|r|c|c|c|}
\hline & $\mu=2$ & $\mu=3$ & $\mu=4$ \\
\hline mean edge length $\xi$ & 2.69 & 2.56 & 2.83 \\
clustering $C$ & 0.0375 & 0.555 & 0.595 \\
hop distance $h$ & 9.31 & 7.98 & 5.74 \\
route distance $r$ & 24.4 & 23.0 & 19.6 \\
Route factor $R$ & 0.653 & 0.525 & 0.268 \\
\hline
\end{tabular}

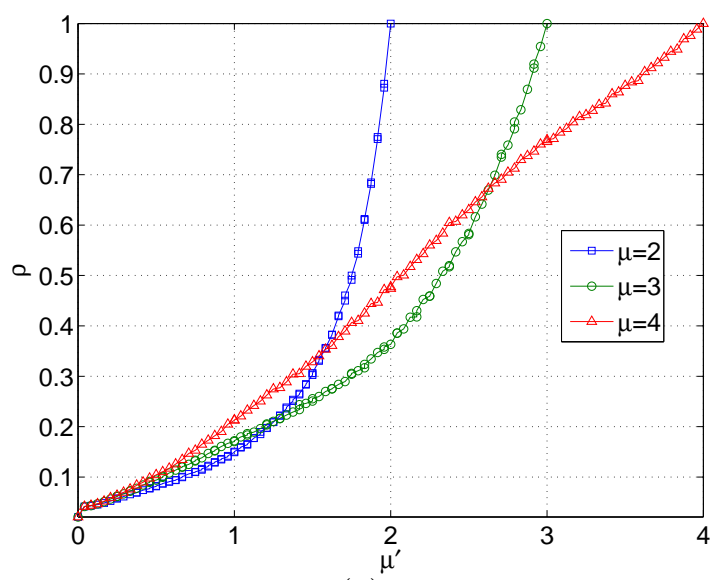

(a)

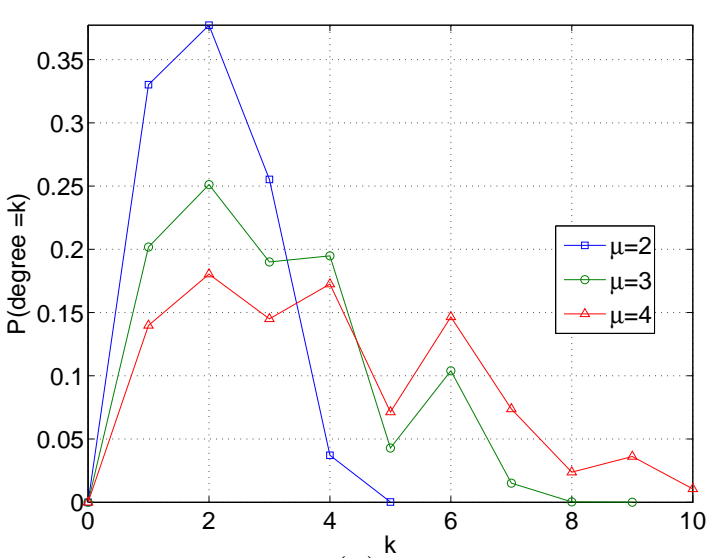

(b)

Figure 4.15: Comparison of (a) robustness and (b) degree distribution, of simulation results for US state capitals' locations data for three values of $\mu$.

Our next test bed for the connected ESNM is the locations of the lower 48 US state capitals. Here, the vertices are more uniformly distributed than in the the road network we considered earlier. Nonetheless, Fig. 4.14(b) shows the accumulation of edges when $\mu=3$, in the north east region where the density of states is high. In Table 4.2 shows that, as is to be expected, the clustering coefficient and all the distance measures decrease when $\mu$ increases. The mean edge length, however, is nonmonotone, consistent with our earlier findings in Fig. 4.9. The robustness profile in Fig. 4.15(a) shows that the $\mu=4$ network is robust by our criterion, while $\mu=2$ and 3 are not, i.e., $3<\mu_{*}<4$. Fig. 4.15 (b) shows the degree distribution; the jaggedness of the $\mu=3$ and 4 curves is peculiar and is perhaps due to the small system size. 


\subsection{Summary and Outlook}

In this paper we have introduced an abstract model for the evolution of spatial networks whose equilibrium distribution is known, and is specified by their spatial dimension, their mean degree, a topological constraint and the inverse temperature $\beta$. We examined two cases - one where the topological constraint is absent, and the other where the network is required to be connected.

The unconstrained network is closely related to a percolation problem. This enabled us to analytically compute the distribution of the degrees and edge lengths, and the clustering coefficient of the network. Other quantities such as the critical mean degree $\mu_{*}^{(\beta \rightarrow \infty)}$ for percolation were estimated by simulation. One interesting aspect of this model is that that it interpolates between the Erdős-Rényi random graph $(\beta=0)$ and the random geometric graph $(\beta=\infty)$. Furthermore, the unconstrained ESNM can be a model for a social network where stubborn individuals with fixed opinions a number of issues, have a tendency to rewire their ties to those with similar opinions. Even when this tendency was high, the fact that the number of edges is fixed, ensured that a small mean degree was enough to have a giant component.

An analytical framework for computing quantities associated with the connected network model is lacking, so we studied that model purely by simulation concentrating on the random connected network $(\beta=0)$ and the almost optimized network $(\beta=10)$. Our analysis focused on the total length (wiring cost) of the network, how routes between vertices compare with their spatial separation, and the robustness of the network to random removal of edges. In the former two aspects, we found that the almost optimized network is notably more efficient than the random connected network. However, in terms of the metric that we proposed, the RCN was found to be more robust. A peculiar feature we noted of the $\operatorname{AON}(\mu)$ is that the mean edge length is the lowest when $\mu \approx 2.2$, and not at 2 as one would expect. 
To test the success of our second model for network design we considered two examples: the Minnesota road network and the 48 state capitals of the continental US. While the rewiring produced networks with good values of some important statistics, additional criteria (e.g., reweighted edges based on population density to compensate for uneven vertex distributions) will need to be introduced to produce good solutions. 


\section{Closing remarks}

The primary motivation for all the models presented in this dissertation has been social networks, and is a common theme that unites them. The evolving voter model and the unconstrained ESNM are models of social networks where individuals actively alter their ties based on their opinions. In the former, there is a single issue on which individuals have one of two incompatible opinions that could change under each others' influence; where as in the latter, there may be many issues with a continuum of possible opinions about each, but individuals stubbornly hold on to their original opinions. The eventual fission of the network is inevitable in the evolving voter model, although its nature depends in a non-trivial way on (1) the relative ease with which new friendships can be created, as compared to influencing one another, and (2) on whether or not the new friends are chosen based on their opinion. Our study provides an explanation for this observation. In the evolving spatial network, however, the disintegration is avoided if the average number of friendships per individual exceeds a minimum number.

The quadratic contact process takes place on a social network that is frozen in time. Hence it is an appropriate model for the spread of fads or rumors that happen 
over a very short time scale, that is insufficient for new friendships to be made or existing ones to be broken. The survival of the fad obviously depends on how contagious it is, but our study shows that the degree distribution of the network too has a significant effect. If the fad or rumor is not sufficiently contagious, it is crucial that the network contains highly connected individuals who act as the primary "sources"; in the absence of hubs, there is a minimum threshold for the contagiousness as well as the seed population of the fad's adopters, if the fad is to survive.

In addition to examining the dynamics of social networks, this dissertation also makes a minor foray into transport and distribution network design, through the connected ESNM. The use of this model to optimize the Minnesota road network, and design a road network to connect the 48 state capitals in the US, shows that the process needs modification in order to be useful for applications. Nonetheless, we believe that the model is interesting from a theoretical standpoint, and warrants further investigation.

All of the models studied here are very simple, as are the Ising model and percolation from statistical mechanics. However, we believe that they offer insights into the behavior of more complicated systems. The evolving voter model and other systems where the network and the states of the nodes coevolve have interesting phase transitions, but they are also relevant to important problems such as the spread of the human pappiloma virus through high school social networks. 


\section{Bibliography}

[1] Mark E. J. Newman. Networks : An Introduction. Oxford University Press, Oxford New York, 2010.

[2] Gerhard Werner. Consciousness viewed in the framework of brain phase space dynamics, criticality, and the renormalization group. Chaos, Solitons 86 Fractals, 55(0):3 - 12, 2013. Emergent Critical Brain Dynamics.

[3] Albert-László Barabási and Eric Bonabeau. Scale-Free Networks. Scientific American, 288:60-69, 2003.

[4] Santo Fortunato. Community detection in graphs. Physics Reports, 486(3):75$174,2010$.

[5] Ithiel de Sola Pool and Manfred Kochen. Contacts and influence. Social Networks, 1(1):5 - 51, 1978-1979.

[6] Ray Solomonoff and Anatol Rapoport. Connectivity of random nets. The bulletin of mathematical biophysics, 13(2):107-117, 1951.

[7] Paul Erdős and Alfréd Rényi. On random graphs, I. Publicationes Mathematicae (Debrecen), 6:290 - 297, 1959.

[8] Duncan J. Watts and Steven H. Strogatz. Collective dynamics of smallworldnetworks. Nature, 393(6684):440-442, 1998.

[9] Albert-László Barabási and Réka Albert. Emergence of scaling in random networks. Science, 286:509-512, 1999.

[10] S. Boccaletti, V. Latora, Y. Moreno, M. Chavez, and D.-U. Hwang. Complex networks: Structure and dynamics. Physics Reports, 424(45):175 - 308, 2006.

[11] Rick Durrett. Random Graph Dynamics. Cambridge University Press, New York, NY, USA, 2006. 
[12] Michael Molloy and Bruce Reed. A critical point for random graphs with a given degree sequence. Random Structures \&3 Algorithms, 6(2-3):161-180, 1995.

[13] Paul Erdős and Alfréd Rényi. On the evolution of random graphs. Magyar Tud. Akad. Mat. Kutató Int. Közl, 5:17-61, 1960.

[14] Paul Erdős and Alfréd Rényi. On the strength of connectedness of a random graph. Acta Mathematica Academiae Scientiarum Hungaricae, 12(1-2):261267, September 1964.

[15] Edgar Nelson Gilbert. Random graphs. The Annals of Mathematical Statistics, 30(4):pp. 1141-1144, 1959.

[16] Dietrich Stauffer and Amnon Aharony. Introduction to percolation theory. Taylor and Francis, 1994.

[17] H. W. Watson and Francis Galton. On the probability of the extinction of families. The Journal of the Anthropological Institute of Great Britain and Ireland, 4:pp. 138-144, 1875.

[18] Edward A. Bender and E. Rodney Canfield. The asymptotic number of labeled graphs with given degree sequences. Journal of Combinatorial Theory, Series A, 24(3):296-307, 1978.

[19] Béla Bollobás. A probabilistic proof of an asymptotic formula for the number of labelled regular graphs. European Journal of Combinatorics, 1(4):311-316, 1980.

[20] Mark E. J. Newman, Steven H. Strogatz, and Duncan J. Watts. Random graphs with arbitrary degree distributions and their applications. Physical Review E, 64(2):026118, 2001.

[21] Michalis Faloutsos, Petros Faloutsos, and Christos Faloutsos. On power-law relationships of the internet topology. SIGCOMM Comput. Commun. Rev., 29(4):251-262, August 1999.

[22] Mark E. J. Newman. Scientific collaboration networks. I. network construction and fundamental results. Phys. Rev. E, 64:016131, Jun 2001.

[23] Albert-László Barabási. Scale-free networks: a decade and beyond. Science, 325(5939):412-413, 2009. 
[24] Remco van der Hofstad and Gerard Hooghiemstra. Universality for distances in power-law random graphs. Journal of Mathematical Physics, 49(12):-, 2008.

[25] Remco van der Hofstad, Gerard Hooghiemstra, and Dmitri Znamenski. Random graphs with arbitrary i.i.d. degrees. ArXiv Mathematics e-prints, February 2005.

[26] H. Eugene Stanley. Scaling, universality, and renormalization: Three pillars of modern critical phenomena. Rev. Mod. Phys., 71:S358-S366, Mar 1999.

[27] Nigel Goldenfeld. Lectures On Phase Transitions And The Renormalization Group (Frontiers in Physics, 85). Westview Press, illustrated edition edition, July 1992.

[28] Leo P. Kadanoff. Statistical Physics: Statics, Dynamics and Renormalization. Statistical Physics: Statics, Dynamics and Renormalization. World Scientific, 2000 .

[29] S. N. Dorogovtsev, A. V. Goltsev, and J. F. F. Mendes. Critical phenomena in complex networks. Rev. Mod. Phys., 80:1275-1335, Oct 2008.

[30] Haye Hinrichsen. Non-equilibrium phase transitions. Physica A: Statistical Mechanics and its Applications, 369(1):1-28, 2006.

[31] T Chou, K Mallick, and RKP Zia. Non-equilibrium statistical mechanics: from a paradigmatic model to biological transport. Reports on progress in physics, 74(11):116601, 2011.

[32] Michael C. Cross. http://www.cmp.caltech.edu/ mcc/BNU/Notes1_2.pdf. unpublished.

[33] David J Murrell, Ulf Dieckmann, and Richard Law. On moment closures for population dynamics in continuous space. Journal of Theoretical Biology, 229(3):421-432, 2004.

[34] Jerome Benoit, Ana Nunes, and M Telo da Gama. Pair approximation models for disease spread. The European Physical Journal B-Condensed Matter and Complex Systems, 50(1-2):177-181, 2006.

[35] Claudio Castellano, Santo Fortunato, and Vittorio Loreto. Statistical physics of social dynamics. Rev. Mod. Phys., 81:591-646, May 2009. 
[36] Richard Durrett, James P. Gleeson, Alun L. Lloyd, Peter J. Mucha, Feng Shi, David Sivakoff, Joshua E. S. Socolar, and Chris Varghese. Graph fission in an evolving voter model. Proceedings of the National Academy of Sciences, 109(10):3682-3687, 2012.

[37] Adam Douglas Henry, Paweł Prałat, and Cun-Quan Zhang. Emergence of segregation in evolving social networks. Proceedings of the National Academy of Sciences, 108(21):8605-8610, 2011.

[38] Sam R. Magura, Vitchyr He Pong, David Sivakoff, and Rick Durrett. Two evolving social network models. http://www. math.duke.edu/ rtd/NCSSM/ evonets.html, 2013.

[39] Chris Varghese and Rick Durrett. Phase transitions in the quadratic contact process on complex networks. Phys. Rev. E, 87:062819, Jun 2013.

[40] Réka Albert and Albert-László Barabási. Statistical mechanics of complex networks. Rev. Mod. Phys., 74:47-97, Jan 2002.

[41] Sergey N Dorogovtsev and Jose FF Mendes. Evolution of networks. Advances in physics, 51(4):1079-1187, 2002.

[42] Mark E. J. Newman, Albert-László Barabási, and Duncan J Watts. The structure and dynamics of networks. Princeton University Press, 2006.

[43] Guido Caldarelli. Scale-Free Networks: Complex Webs in Nature and Technology. Number 9780199211517 in OUP Catalogue. Oxford University Press, 2007.

[44] Reuven Cohen and Shlomo Havlin. Complex networks: structure, robustness and function. Cambridge University Press, 2010.

[45] Alain Barrat, Marc Barthlemy, and Alessandro Vespignani. Dynamical Processes on Complex Networks. Cambridge University Press, New York, NY, USA, 2008.

[46] Mark E. J. Newman. Spread of epidemic disease on networks. Phys. Rev. E, 66:016128, Jul 2002.

[47] Erik Volz. SIR dynamics in random networks with heterogeneous connectivity. Journal of Mathematical Biology, 56:293-310, 2008. 
[48] Joel C. Miller. A note on a paper by Erik Volz: SIR dynamics in random networks. Journal of Mathematical Biology, 62(3):349-358, 2011.

[49] Vishal Sood and Sidney Redner. Voter model on heterogeneous graphs. Phys. Rev. Lett., 94:178701, May 2005.

[50] Duncan J. Watts. A simple model of global cascades on random networks. Proceedings of the National Academy of Sciences, 99(9):5766-5771, 2002.

[51] James P. Gleeson and Diarmuid J. Cahalane. Seed size strongly affects cascades on random networks. Phys. Rev. E, 75:056103, May 2007.

[52] Andrea Montanari and Amin Saberi. The spread of innovations in social networks. Proceedings of the National Academy of Sciences, 107(47):20196-20201, 2010 .

[53] Hisashi Ohtsuki, Christoph Hauert, Erez Lieberman, and Martin A. Nowak. A simple rule for the evolution of cooperation on graphs and social networks. Nature, 441(7092):502-505, 2006.

[54] Easley David and Kleinberg Jon. Networks, Crowds, and Markets: Reasoning About a Highly Connected World. Cambridge University Press, New York, NY, USA, 2010.

[55] Thilo Gross and Bernd Blasius. Adaptive coevolutionary networks: a review. J. R. Soc. Interface, 5(20):259-271, 2008.

[56] Brian Skyrms and Robin Pemantle. A dynamic model of social network formation. 97(16):9340-9346, 2000.

[57] Holger Ebel and Stefan Bornholdt. Coevolutionary games on networks. Phys. Rev. E, 66:056118, Nov 2002.

[58] Martín G. Zimmermann, Víctor M. Eguíluz, and Maxi San Miguel. Coevolution of dynamical states and interactions in dynamic networks. Phys. Rev. E, 69:065102, Jun 2004.

[59] Martín G. Zimmermann and Víctor M. Eguíluz. Cooperation, social networks, and the emergence of leadership in a prisoner's dilemma with adaptive local interactions. Phys. Rev. E, 72:056118, Nov 2005. 
[60] Francisco C. Santos, Jorge M. Pacheco, and Tom Lenaerts. Cooperation prevails when individuals adjust their social ties. PLoS computational biology, 2(10):e140, 2006.

[61] Erik Volz and Lauren Ancel Meyers. Susceptible-infected-recovered epidemics in dynamic contact networks. Proceedings of the Royal Society B: Biological Sciences, 274(1628):2925-2934, 2007.

[62] Erik Volz and Lauren Ancel Meyers. Epidemic thresholds in dynamic contact networks. Journal of the Royal Society Interface, 6(32):233-241, 2009.

[63] Thilo Gross, Carlos J. Dommar D'Lima, and Bernd Blasius. Epidemic dynamics on an adaptive network. Phys. Rev. Lett., 96:208701, May 2006.

[64] Damian H Zanette. Coevolution of agents and networks in an epidemiological model. arXiv preprint arXiv:0707.1249, 2007.

[65] Sven Van Segbroeck, Francisco C. Santos, and Jorge M. Pacheco. Adaptive contact networks change effective disease infectiousness and dynamics. PLoS Computational Biology, 6(8):e1000895, 2010.

[66] Santiago Gil and Damián H. Zanette. Coevolution of agents and networks: Opinion spreading and community disconnection. Physics Letters A, 356(2):89$94,2006$.

[67] Damián H. Zanette and Santiago Gil. Opinion spreading and agent segregation on evolving networks. Physica D: Nonlinear Phenomena, 224(1):156-165, 2006.

[68] Jorge M. Pacheco, Arne Traulsen, and Martin A. Nowak. Coevolution of strategy and structure in complex networks with dynamical linking. Physical Review Letters, 97(25):258103, 2006.

[69] Balazs Kozma and Alain Barrat. Consensus formation on coevolving networks: groups' formation and structure. Journal of Physics A: Mathematical and Theoretical, 41(22):224020, 2008.

[70] Balazs Kozma and Alain Barrat. Consensus formation on adaptive networks. Phys. Rev. E, 77:016102, Jan 2008.

[71] Gerardo Iñiguez, János Kertész, Kimmo K Kaski, and R. A. Barrio. Opinion and community formation in coevolving networks. Physical Review E, 80(6):066119, 2009. 
[72] Michael W. Macy, James A. Kitts, Andreas Flache, and Steve Benard. Polarization in dynamic networks: A hopfield model of emergent structure. Dynamic Social Network Modeling and Analysis, pages 162-173, 2003.

[73] Petter Holme and Mark E. J. Newman. Nonequilibrium phase transition in the coevolution of networks and opinions. Physical Review E, 74(5):056108, 2006.

[74] Daichi Kimura and Yoshinori Hayakawa. Coevolutionary networks with homophily and heterophily. Phys. Rev. E, 78:016103, Jul 2008.

[75] Federico Vazquez, Víctor M. Eguíluz, and Maxi San Miguel. Generic absorbing transition in coevolution dynamics. Phys. Rev. Lett., 100:108702, Mar 2008.

[76] Jose Luis Herrera, Mario G. Cosenza, K. Tucci, and Juan Carlos GonzálezAvella. General coevolution of topology and dynamics in networks. EPL (Europhysics Letters), 95(5):58006, 2011.

[77] Rick Durrett. Probability: Theory and Examples, volume 3. Cambridge university press, 2010.

[78] Richard A. Holley and Thomas M. Liggett. Ergodic theorems for weakly interacting infinite systems and the voter model. The Annals of Probability, pages 643-663, 1975.

[79] Thomas M. Liggett. Stochastic interacting systems: contact, voter and exclusion processes, volume 324. Springer, 1999.

[80] J. Theodore Cox and A. Greven. On the long term behavior of some finite particle systems. Probability theory and related fields, 85(2):195-237, 1990.

[81] Mark E. J. Newman. The Structure and Function of Complex Networks. SIAM Review, 45:167-256, January 2003.

[82] Colin Cooper and Alan Frieze. A general model of web graphs. Random Struct. Algorithms, 22(3):311-335, May 2003.

[83] Romualdo Pastor-Satorras and Alessandro Vespignani. Epidemic dynamics and endemic states in complex networks. Phys. Rev. E, 63:066117, May 2001.

[84] Noam Berger, Christian Borgs, Jennifer T. Chayes, and Amin Saberi. On the spread of viruses on the internet. In Proceedings of the sixteenth annual ACM-SIAM symposium on Discrete algorithms, SODA '05, pages 301-310, Philadelphia, PA, USA, 2005. Society for Industrial and Applied Mathematics. 
[85] Thomas M. Liggett. Interacting particle systems. Springer-Verlag, New York, 1985.

[86] Theodore Edward Harris. Contact interactions on a lattice. The Annals of Probability, 2(6):969-988, 1974.

[87] Malte Henkel, Haye Hinrichsen, and Sven Lübeck. Non-Equilibrium Phase Transitions: Volume 1: Absorbing Phase Transitions, volume 1. Springer, 2009 .

[88] Joaquín Marro and Ronald Dickman. Nonequilibrium phase transitions in lattice models. Cambridge University Press, 2005.

[89] Andrei L. Toom. Stable and attractive trajectories in multicomponent systems. Multicomponent Systems, 6:549-575, 1980.

[90] Hwa-Nien Chen. On the stability of a population growth model with sexual reproduction on $\mathbb{Z}^{2}$. The Annals of Probability, 20(1):232-285, 1992.

[91] Hwa-Nien Chen. On the stability of a population growth model with sexual reproduction on $\mathbb{Z}^{d}, d \geq 2$. The Annals of Probability, 22(3):1195-1226, 1994.

[92] Xiaofang Guo, Da-Jiang Liu, and James W. Evans. Generic two-phase coexistence, relaxation kinetics, and interface propagation in the quadratic contact process: Simulation studies. Phys. Rev. E, 75:061129, Jun 2007.

[93] Da-Jiang Liu, Xiaofang Guo, and James W. Evans. Quadratic contact process: Phase separation with interface-orientation-dependent equistability. Phys. Rev. Lett., 98:050601, Feb 2007.

[94] Xiaofang Guo, James W. Evans, and Da-Jiang Liu. Generic two-phase coexistence, relaxation kinetics, and interface propagation in the quadratic contact process: Analytic studies. Physica A: Statistical Mechanics and its Applications, 387(1):177 - 201, 2008.

[95] Xiaofang Guo, Da-Jiang Liu, and James W. Evans. Schloegl's second model for autocatalysis with particle diffusion: Lattice-gas realization exhibiting generic two-phase coexistence. The Journal of Chemical Physics, 130(7):074106, 2009.

[96] Da-Jiang Liu. Generic two-phase coexistence and nonequilibrium criticality in a lattice version of schlgls second model for autocatalysis. Journal of Statistical Physics, 135:77-85, 2009. 
[97] Friedrich Schlögl. Chemical reaction models for non-equilibrium phase transitions. Zeitschrift für Physik A Hadrons and Nuclei, 253(2):147-161, 1972.

[98] Peter Grassberger. On phase transitions in Schlögl's second model. Zeitschrift für Physik B Condensed Matter, 47:365-374, 1982.

[99] Shirshendu Chatterjee and Rick Durrett. Contact processes on random graphs with power law degree distributions have critical value 0. Ann. Probab., 37(6):2332-2356, 2009.

[100] Roni Parshani, Shai Carmi, and Shlomo Havlin. Epidemic threshold for the susceptible-infectious-susceptible model on random networks. Phys. Rev. Lett., 104:258701, Jun 2010.

[101] Damon Centola and Michael Macy. Complex contagions and the weakness of long ties. American Journal of Sociology, 113(3):702-734, 2007.

[102] Shirshendu Chatterjee and Rick Durrett. A first order phase transition in the threshold contact process on random $r$-regular graphs and $r$-trees. Stochastic Processes and their Applications, 123(2):561 - 578, 2013.

[103] G. J. Baxter, S. N. Dorogovtsev, A. V. Goltsev, and J. F. F. Mendes. Bootstrap percolation on complex networks. Phys. Rev. E, 82:011103, Jul 2010.

[104] Thomas Mountford, Daniel Valesin, and Qiang Yao. Metastable densities for the contact process on power law random graphs. Electron. J. Probab., 18:no. 103, 1-36, 2013.

[105] Thomas Mountford, Jean-Christophe Mourrat, Daniel Valesin, and Qiang Yao. Exponential extinction time of the contact process on finite graphs. ArXiv eprints, March 2012.

[106] L. R. G. Fontes and R. H. Schonmann. Bootstrap Percolation on Homogeneous Trees Has 2 Phase Transitions. Journal of Statistical Physics, 132:839-861, September 2008.

[107] József Balogh and Boris G. Pittel. Bootstrap percolation on the random regular graph. Random Structures \& Algorithms, 30(1-2):257-286, 2007.

[108] Richard Durrett. On the growth of one dimensional contact processes. The Annals of Probability, 8(5):890-907, 1980. 
[109] S. Prakash and G. Nicolis. Dynamics of the Schlögl models on lattices of low spatial dimension. Journal of Statistical Physics, 86:1289-1311, 1997.

[110] Carol Bezuidenhout and Geoffrey Grimmett. The critical contact process dies out. The Annals of Probability, 18(4):1462-1482, 1990.

[111] Gregory J. Morrow, Rinaldo B. Schinazi, and Yu Zhang. The critical contact process on a homogeneous tree. Journal of Applied Probability, 31(1):250-255, 1994.

[112] Robin Pemantle. The contact process on trees. The Annals of Probability, 20(4):2089-2116, 1992.

[113] Feng Xie and David Levinson. Modeling the Growth of Transportation Networks: A Comprehensive Review. Networks and Spatial Economics, 9(3):291307, October 2007.

[114] David J. Aldous. Optimal spatial transportation networks where link costs are sublinear in link capacity. Journal of Statistical Mechanics: Theory and Experiment, 2008(03):P03006, March 2008.

[115] David J. Aldous. Spatial transportation networks with transfer costs: asymptotic optimality of hub-and-spoke models. Mathematical Proceedings of the Cambridge Philosophical Society, 145(02):471-487, April 2008.

[116] Atsushi Tero, Seiji Takagi, Tetsu Saigusa, Kentaro Ito, Dan P Bebber, Mark D Fricker, Kenji Yumiki, Ryo Kobayashi, and Toshiyuki Nakagaki. Rules for biologically inspired adaptive network design. Science, 327(5964):439-42, January 2010 .

[117] Rémi Louf, Pablo Jensen, and Marc Barthelemy. Emergence of hierarchy in cost-driven growth of spatial networks. Proceedings of the National Academy of Sciences of the United States of America, 110(22):8824-9, May 2013.

[118] Michael T. Gastner and Mark E. J. Newman. Shape and efficiency in spatial distribution networks. Journal of Statistical Mechanics: Theory and Experiment, 2006(01):P01015-P01015, January 2006.

[119] Gerald F. Frasco, Jie Sun, Hernán D. Rozenfeld, and Daniel Ben-Avraham. Spatially Distributed Social Complex Networks. Physical Review $X$, 4(1):011008, January 2014. 
[120] Ed Bullmore and Olaf Sporns. Complex brain networks: graph theoretical analysis of structural and functional systems. Nature reviews. Neuroscience, 10(3):186-98, March 2009.

[121] Ed Bullmore and Olaf Sporns. The economy of brain network organization. Nature reviews. Neuroscience, 13(5):336-49, May 2012.

[122] Danielle S. Bassett, Nicholas F. Wymbs, Mason A. Porter, Peter J. Mucha, Jean M. Carlson, and Scott T. Grafton. Dynamic reconfiguration of human brain networks during learning. Proceedings of the National Academy of Sciences of the United States of America, 108(18):7641-6, May 2011.

[123] Marc Barthélemy. Spatial networks. Physics Reports, 499(1-3):1-101, February 2011.

[124] B.M. Waxman. Routing of multipoint connections. IEEE Journal on Selected Areas in Communications, 6(9):1617-1622, 1988.

[125] L. Barnett, E. Di Paolo, and S. Bullock. Spatially embedded random networks. Physical Review E, 76(5):056115, November 2007.

[126] Amir Minerbi, Roni Kahana, Larissa Goldfeld, Maya Kaufman, Shimon Marom, and Noam E Ziv. Long-term relationships between synaptic tenacity, synaptic remodeling, and network activity. PLoS biology, 7(6):e1000136, June 2009.

[127] Nicholas Metropolis, Arianna W. Rosenbluth, Marshall N. Rosenbluth, Augusta H. Teller, and Edward Teller. Equation of State Calculations by Fast Computing Machines. The Journal of Chemical Physics, 21(6):1087, December 1953.

[128] Raj Kumar Pathria and Paul D. Beale. Statistical Mechanics, Third Edition. Academic Press, 2007.

[129] Jesper Dall and Michael Christensen. Random geometric graphs. Physical Review E, 66(1):016121, July 2002.

[130] Mathew Penrose. Random Geometric Graphs (Oxford Studies in Probability). Oxford University Press, USA, 2003.

[131] Alain Barrat, Marc Barthélemy, and Alessandro Vespignani. The effects of spatial constraints on the evolution of weighted complex networks. Journal of Statistical Mechanics: Theory and Experiment, 2005(05):P05003, May 2005. 
[132] My T. Thai and Panos Pardalos. Handbook of Optimization in Complex Networks, volume 57 of Springer Optimization and Its Applications. Springer US, Boston, MA, 2012.

[133] R.L. Graham and Pavol Hell. On the History of the Minimum Spanning Tree Problem. IEEE Annals of the History of Computing, 7(1):43-57, 1985.

[134] R. C. Prim. Shortest Connection Networks And Some Generalizations. Bell System Technical Journal, 36(6):1389-1401, November 1957.

[135] David J. Aldous and Wilfrid S. Kendall. Short-length routes in low-cost networks via Poisson line patterns. Advances in Applied Probability, 40(1):1-21, March 2008.

[136] David J. Aldous and Julian Shun. Connected Spatial Networks over Random Points and a Route-Length Statistic. Statistical Science, 25(3):275-288, August 2010 .

[137] Shan He, Sheng Li, and Hongru Ma. Effect of edge removal on topological and functional robustness of complex networks. Physica A: Statistical Mechanics and its Applications, 388(11):2243-2253, June 2009.

[138] http://www.cise.ufl.edu/research/sparse/matrices/Gleich/ minnesota.html. Accessed on 04/25/2014. 


\section{Biography}

Chris Varghese was born on April 29, 1985 in Trivandrum, the capital city of the state of Kerala in southern India, where he also did his schooling. He received a B.Tech degree in Engineering Physics from Indian Institute of Technology Bombay in 2008, followed by M.S. and Ph.D degrees in Physics from Mississippi State University in 2010 and Duke University in 2014, respectively. His research work has mostly been on networks. 